Aus der Klinik für Augenheilkunde

(Prof Dr. med H. Hoerauf)

der Medizinischen Fakultät der Universität Göttingen

\title{
Mittelfristiger Einfluss der Vitrektomie bei Makulaerkrankungen auf den Augeninnendruck
}

\author{
INAUGURAL-DISSERTATION \\ zur Erlangung des Doktorgrades \\ der Medizinischen Fakultät der Georg-August-Universität zu Göttingen \\ vorgelegt von \\ Julia Charlotte Gebest \\ aus \\ Heidelberg
}

Göttingen 2017 
Dekan:

Referent/in:

Ko-Referent/in:
Prof. Dr. rer. nat. H. K. Kroemer

PD Dr. med. C. van Oterendorp

PD Dr. Dr. A. Meyer

Datum der mündlichen Prüfung: 24.01.2019 
Hiermit erkläre ich, die Dissertation mit dem Titel „Mittelfristiger Einfluss der Vitrektomie bei Makulaerkrankungen auf den Augeninnendruck“ eigenständig angefertigt und keine anderen als die von mir angegebenen Quellen und Hilfsmittel verwendet zu haben.

Göttingen, den

(Unterschrift) 


\section{Inhaltsverzeichnis}

Abkürzungsverzeichnis ……………………………………………………………….. IV

Abbildungsverzeichnis ............................................................................................. VI

Tabellenverzeichnis........................................................................................................ VII

Definition der berechneten Druckänderungen ................................................................. X

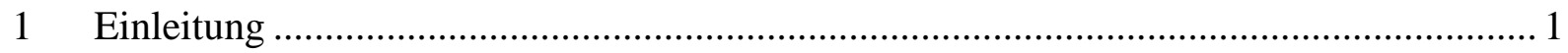

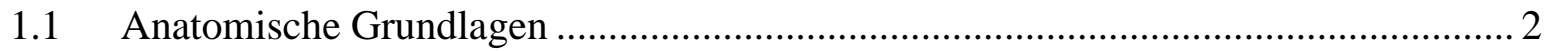

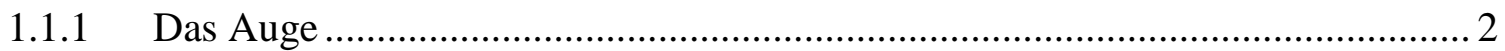

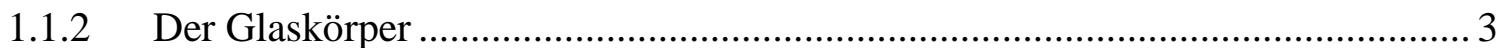

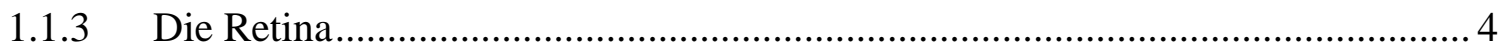

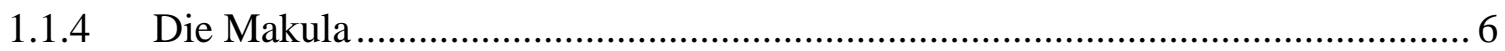

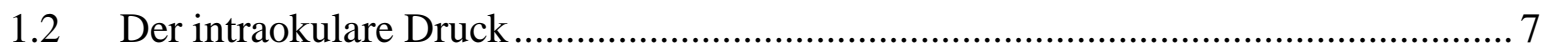

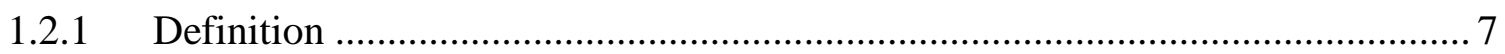

1.2.2 Regulation des intraokularen Drucks ............................................................ 7

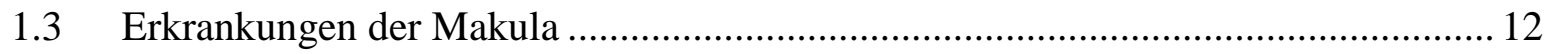

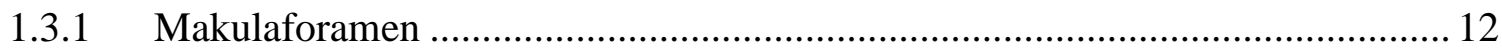

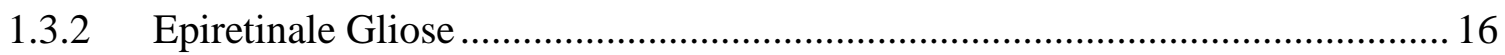

1.3.3 Vitreomakuläres Traktionssyndrom …………………………………………... 18

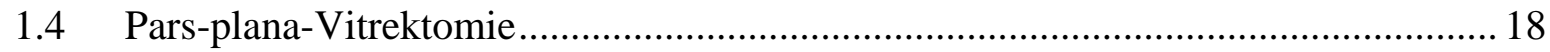

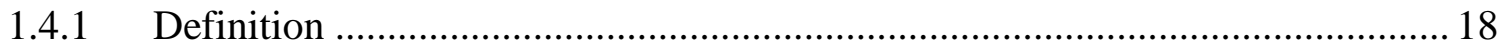

1.4.2 Geschichte der Vitrektomie........................................................................ 18

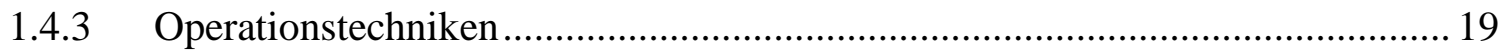

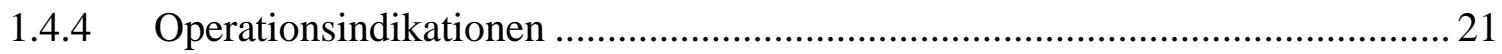

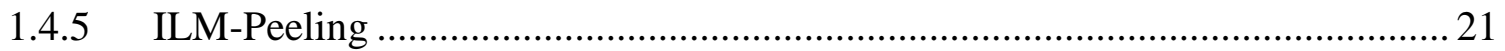

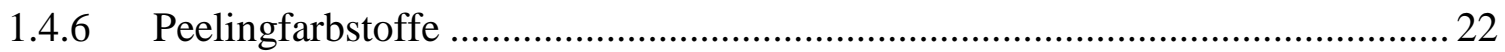




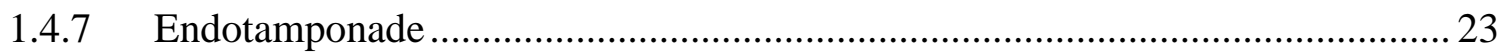

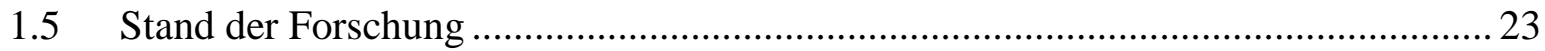

1.5.1 IOD-Entwicklung unmittelbar postoperativ .............................................. 23

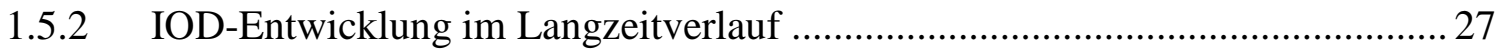

1.5.3 Einfluss der Vitrektomgröße (20 G, 23 G) auf den IOD................................. 31

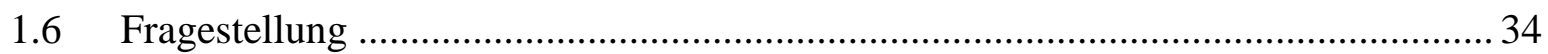

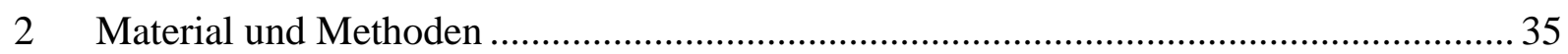

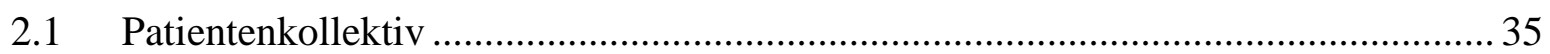

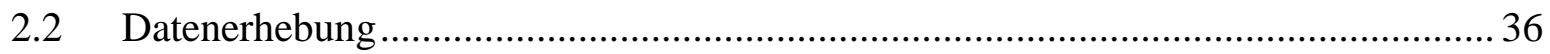

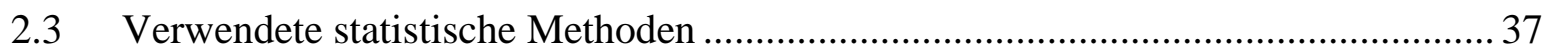

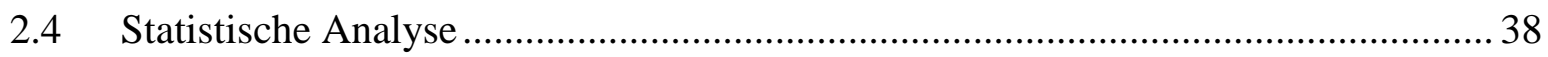

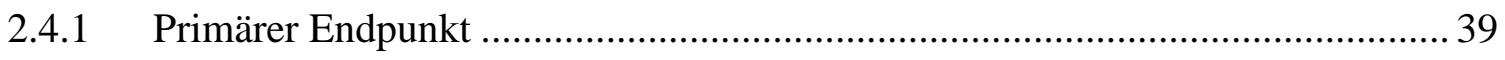

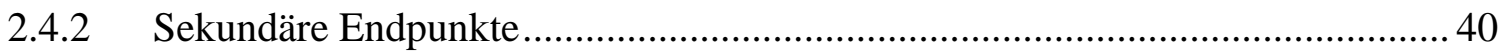

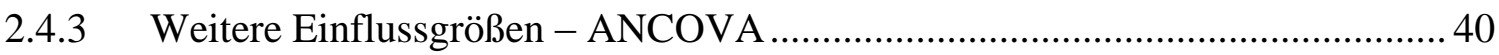

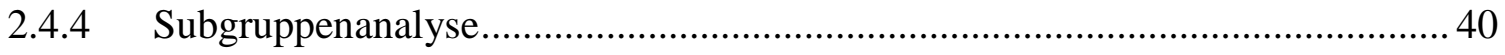

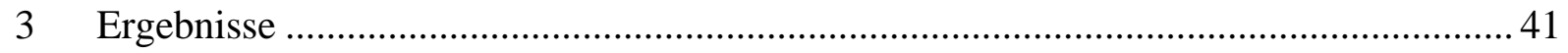

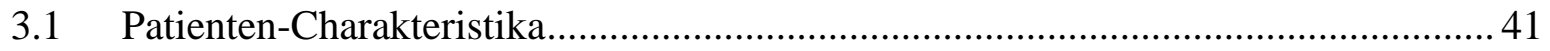

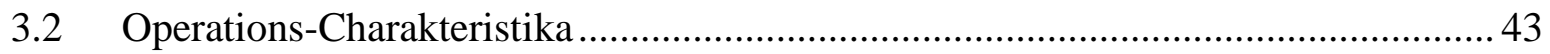

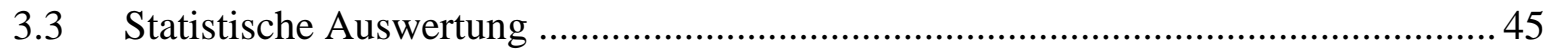

3.3.1 Augeninnnendruck: Übersicht der Daten ................................................... 45

3.3.2 Analyse des primären Endpunktes: IOD-Änderung nach 6-12 Monaten .......... 49

3.3.3 Analyse sekundärer Endpunkte; Teil 1: IOD-Änderung nach 3-6 Monaten ..... 53

3.3.4 Analyse sekundärer Endpunkte; Teil 2: IOD-Änderung nach 12-24 Monaten. 55

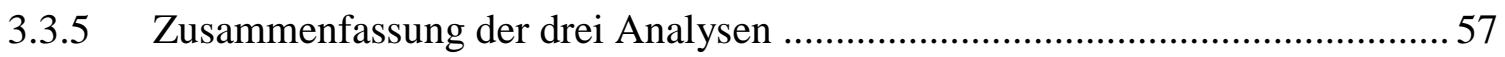

3.3.6 Einflussfaktoren auf den IOD über ANCOVA-Analyse ................................... 57

3.3.7 Einflussfaktoren auf den IOD über ANCOVA-Analyse mittels LOCF.............59

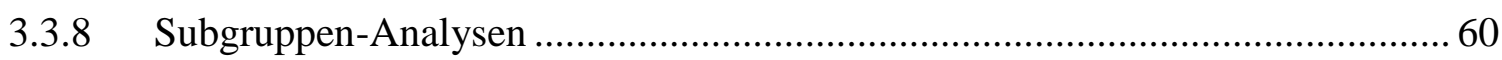


4 Diskussion

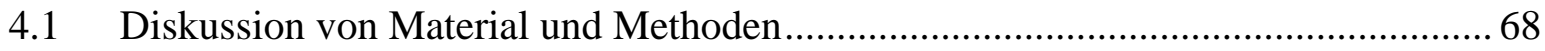

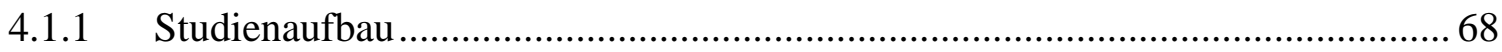

4.1.2 Limitierende Faktoren des Studiendesigns ................................................. 70

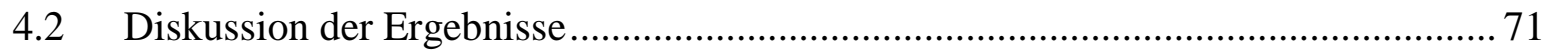

4.2.1 Ergebnisse im Vergleich zum aktuellen Stand der Forschung........................... 71

4.2.2 Diskussion der Einflussfaktoren der Subgruppenanalyse ................................ 74

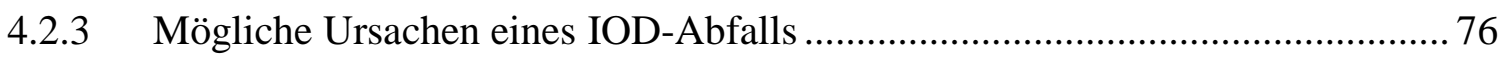

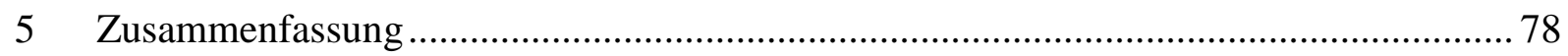

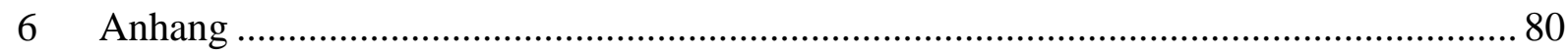

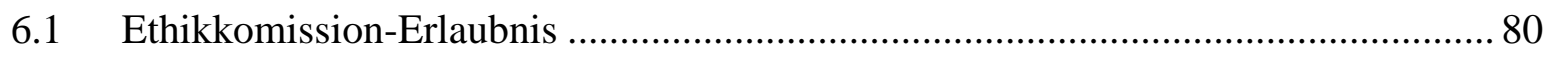

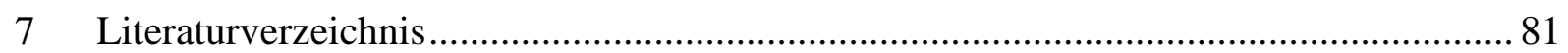




\section{Abkürzungsverzeichnis}

\begin{tabular}{|c|c|}
\hline AT & Augentropfen \\
\hline $\mathrm{BB}$ & Brilliant Blue \\
\hline $\mathrm{BP}$ & Brilliant Peel \\
\hline C2F6 & Hexaflourethan \\
\hline C3F8 & Perfluoropropan \\
\hline CMR & cellophane macular reflex (Zellophanmakulopathie) \\
\hline $\mathrm{D}$ & Dioptrien \\
\hline DB & Dual Blue \\
\hline EG & epiretinale Gliose \\
\hline FU & Follow-up \\
\hline $\mathrm{G}$ & Gauge \\
\hline ICG & Indocyaningrün \\
\hline ILM & Internal/Inner limiting membrane (innere Grenzmembran) \\
\hline ILMB & Internal limiting membrane-Blau \\
\hline ILM-Peeling & Internal limiting membrane-Peeling \\
\hline IOD & intraokularer Druck \\
\hline LCL & lower confidence limit (Untergrenze des Konfidenzintervalls) \\
\hline MAX & Maximum \\
\hline MB & Methylenblau \\
\hline Mean & Mittelwert \\
\hline MF & Makulaforamen \\
\hline MIN & Minimum \\
\hline $\mathrm{N}$ & Stichprobenumfang/Anzahl \\
\hline OCT & optische Kohärenztomographie \\
\hline
\end{tabular}


post-OP postoperativ

prä-OP präoperativ

ppV Pars-plana-Vitrektomie

RPE retinales Pigmentepithel

SÄ $\quad$ sphärisches Äquivalent

SEM Standard Error of the Mean (Standardfehler)

SF6 Sulfur-Hexafluoride

SD Standardabweichung

TB Trypanblau

TGF- 3 Transforming growth factor beta (Wachstumsfaktor)

TMW Trabekelmaschenwerk

UCL upper confidence limit (Obergrenze des Konfidenzintervalls)

VMT vitreomakuläres Traktionssyndrom 


\section{Abbildungsverzeichnis}

Abbildung 1: Übersicht Auge und seine Schichten (Grehn 2008) ....................................... 3

Abbildung 2: Schematischer Aufbau der Retina (Sachsenweger 2003) ................................. 5

Abbildung 3: Schematische Darstellung des trabekulären Abflusses (Goel et al. 2010)........... 9

Abbildung 4: Schematische Darstellung des uveoskleralen Abflusses (Goel et al. 2010) ...... 10

Abbildung 5: Pathomechanismus und Stadieneinteilung des Makulaforamens nach Gass

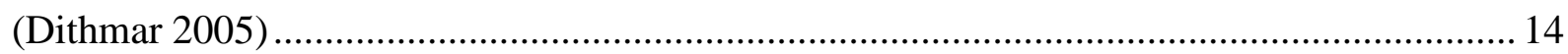

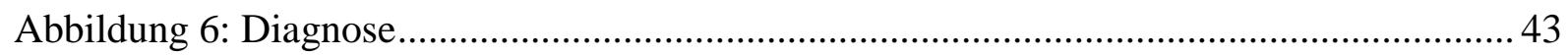

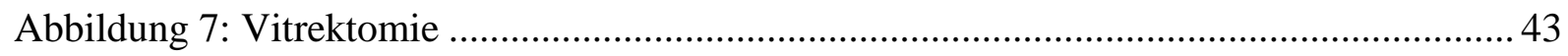

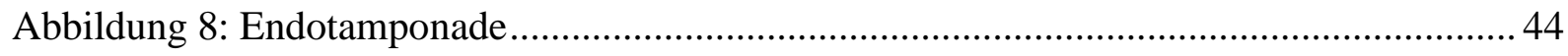

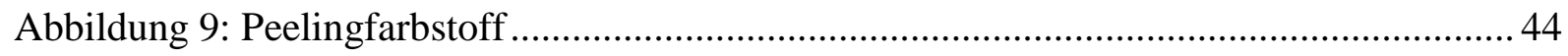

Abbildung 10: Punktwolkendiagramm aller IOD-Werte für das operierte Auge mit Mittelwert und SD.

Abbildung 11 : Verbundene t-Tests des IOD von prä-OP zu definierten Zeitintervallen operiertes Auge mit SD

Abbildung 12: Punktwolkendiagramm aller IOD-Werte für das nicht-operierte Auge mit Mittelwert und SD.

Abbildung 13: Verbundene t-Tests des IOD von prä-OP zu definierten Zeitintervallen nichtoperiertes Auge mit SD 48

Abbildung 14: Vergleich der IOD-Mittelwerte beider Augen zu unterschiedlichen Untersuchungszeitpunkten mit SD 49

Abbildung 15: Verteilungskurve der IOD-Änderung beider Augen für die primäre Analyse. 50 Abbildung 16: Differenz der IOD-Änderung der primären Analyse 51

Abbildung 17: Korrelation der IOD-Werte prä-OP mit IOD-Werten zwischen 6-12 Monate 52 Abbildung 18: Verteilungskurve der IOD-Änderung beider Augen für den sekundären Endpunkt 3-6 Monate .53 
Abbildung 19: Verteilungskurve der IOD-Änderungen beider Augen für den sekundären

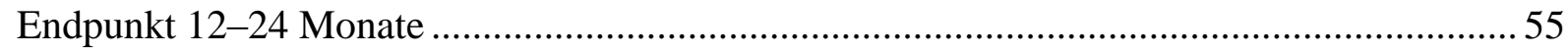

Abbildung 20: Mittelwerte der IOD-Änderungen für die drei Analysen beider Augen .......... 57

Abbildung 21: IOD-Änderung für jeweils $20 \mathrm{G}$ und $23 \mathrm{G}$ des operierten Auges.................... 60

Abbildung 22: Unverbundene t-Tests für die Vitrektomgröße des operierten Auges 61

Abbildung 23: Korrelation der IOD-Werte zwischen präoperativen und anderen definierten

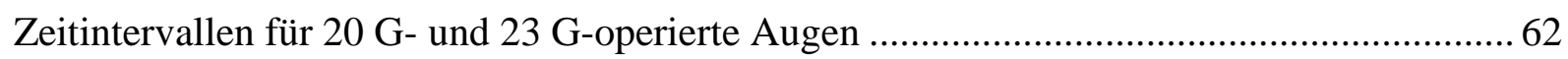

Abbildung 24: IOD-Änderung für jeweils $20 \mathrm{G}$ und $23 \mathrm{G}$ des nicht-operierten Auges .......... 63

Abbildung 25: Unverbundene t-Tests für die Vitrektomie des nicht-operierten Auges .......... 64

Abbildung 26: IOD-Änderung Endotamponaden: Zeitraum 3-6 Monate ............................. 65

Abbildung 27: IOD-Änderung Endotamponaden: Zeitraum 6-12 Monate ............................. 66

Abbildung 28: IOD-Änderung Endotamponaden: Zeitraum 12-24 Monate ............................ 66 


\section{Tabellenverzeichnis}

Tabelle 1: Gauge und korrespondierende Außendurchmesser der Kanüle ............................ 20

Tabelle 2: Verweildauer und Eigenschaften intraokularer Gase (Müller et al. 2010) ............. 23

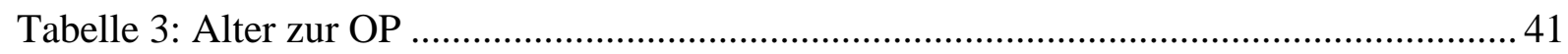

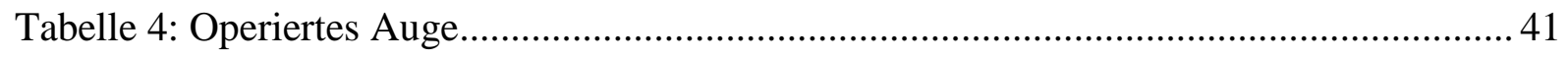

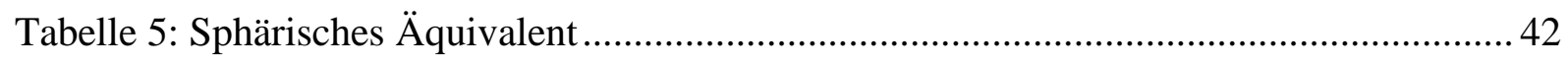

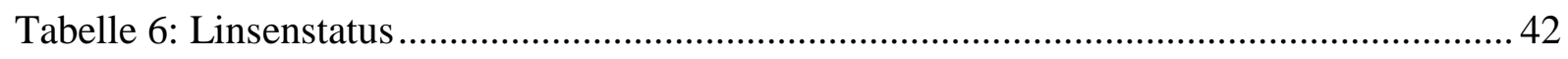

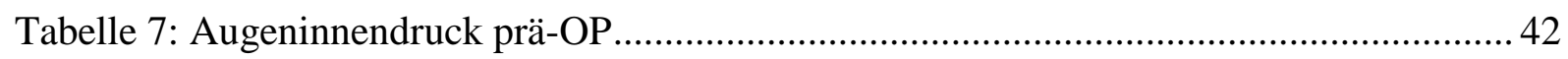

Tabelle 8: IOD des operierten Auges zu verschiedenen Zeitpunkten ................................. 46

Tabelle 9: IOD des nicht-operierten Auges zu verschiedenen Zeitpunkten........................... 48

Tabelle 10: Statistische Auswertung der IOD-Änderung der primären Analyse ..................... 50

Tabelle 11: Auswertung der Differenz der IOD-Änderung für die primäre Analyse .............. 51

Tabelle 12: Statistische Auswertungen des primären Endpunkts mittels LOCF .....................52

Tabelle 13: Statistische Auswertung der IOD-Änderung für den sekundären Endpunkt 3-6

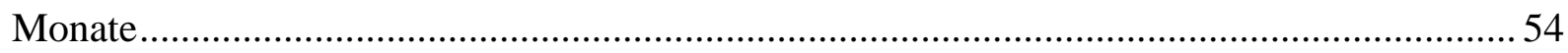

Tabelle 14: Differenz der IOD-Änderung für den sekundären Endpunkt 3-6 Monate............ 54

Tabelle 15: Statistische Auswertungen des sekundären Endpunkts mittels LOCF für den

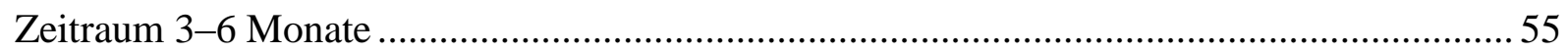

Tabelle 16: Statistische Auswertung der IOD-Differenz, sekundärer Endpunkt 12 bis 24

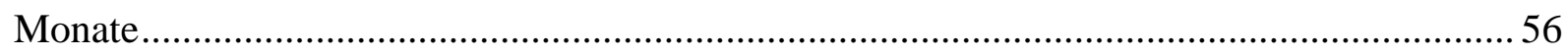

Tabelle 17: Differenz der IOD-Änderung, sekundärer Endpunkt 12-24 Monate.....................56

Tabelle 18: Statistische Auswertungen des sekundären Endpunkts mittels LOCF für den

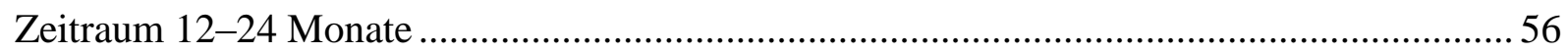

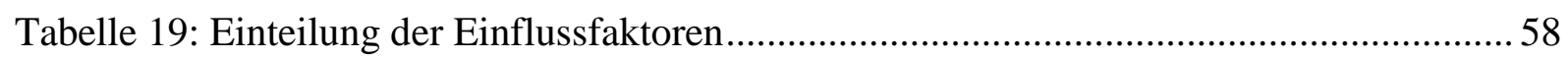

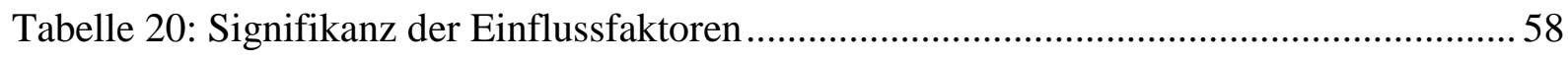

Tabelle 21: Statistische Auswertung der IOD-Änderung des operierten Auges .......................61 
Tabelle 22: Statistische Auswertung der IOD-Änderung des nicht-operierten Auges 63

Tabelle 23: Statistische Auswertung der Differenz der IOD-Änderung ................................ 65

Tabelle 24: Übersicht Vitrektomgröße und jeweilige Endotamponade ................................. 66 


\section{Definition der berechneten Druckänderungen}

IOD-Änderung $=\mathrm{IOD}_{\text {definiertes Zeitintervall }}-\mathrm{IOD}_{\text {prä-OP }}$

Die IOD-Änderung ist die Differenz zwischen den IOD-Werten der jeweilig definierten Zeitintervalle postoperativ (d. h. 3-6 Monate, 6-12 Monate, 12-24 Monate) und den präoperativen IOD-Werten. Wenn also die IOD-Änderung negativ ist, war der Druck bis zum definierten Zeitintervall gesunken $\left(\mathrm{IOD}_{\text {definiertes Zeitintervall }}<\mathrm{IOD}_{\text {prä-OP }}=\right.$ negative IODÄnderung).

Differenz der IOD-Änderung = IOD-Änderung nicht operiert - IOD-Änderung $_{\text {operiert }}$ Für die Differenz der IOD-Änderung wurde die IOD-Änderung des nicht operierten Auges von der IOD-Änderung des operierten Auges subtrahiert. Positive Werte der Subtraktion ergaben sich, wenn die IOD-Änderung (negativ) des operierten Auges größer waren als die des nicht operierten Auges. (IOD-Änderung nicht operiert $_{\text {L IOD-Änderung }}$ operiert $_{1}=$ positiv, wenn

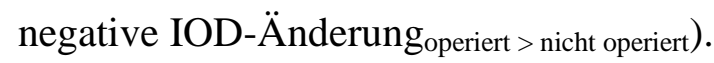




\section{Einleitung}

Makulaforamen und epiretinale Gliose gehören zur Gruppe der Makuladegenerationen und sind am Augenhintergrund in der Mitte der Retina lokalisiert. Da die Makula den Bereich des schärfsten Sehens darstellt, ist es besonders wichtig, Krankheiten, die sie betreffen, besser zu verstehen und zu erforschen. Der Verlust des zentralen (scharfen) Sehens stellt eine massive Einschränkung für betroffene Patienten dar. Aus diesem Grund gibt es zahlreiche Studien, die versuchen, die Entstehung solcher Makuladegenerationen zu verstehen und zu behandeln.

Seit der Erstbeschreibung der Vitrektomie durch Machemer 1971 können die Erkrankungen des Augenhintergrundes besser verstanden und therapiert werden. Dabei handelt es sich um ein Operationsverfahren, bei dem der Glaskörper entfernt und durch physiologische Salzlösung, Gas oder Öl ersetzt wird. Die Vitrektomie über Pars-plana-Zugang (ppV) mit kombinierter Entfernung der inneren Grenzmembran der Netzhaut, das sogenannte ILM-Peeling, etablierte sich als therapeutisches Verfahren für Operationen des Makulaforamens und epiretinaler Gliose.

Im Gegensatz zu den Makulaerkrankungen wird beim Glaukom, einer Degeneration des Sehnervs (Nervus opticus), das periphere Sehen irreversibel beeinträchtigt. Hauptrisikofaktor für die Entstehung eines Glaukoms ist ein erhöhter intraokularer Druck. Die Therapie besteht in einer Drucksenkung, entweder mittels Augentropfen oder durch drucksenkende Operationen.

Aufgrund klinischer Beobachtungen nach zahlreichen Vitrektomien wird als Annahme und gleichzeitig als Grundlage dieser Dissertation die Hypothese formuliert, dass der intraokulare Druck (IOD) nach Vitrektomie zwar unmittelbar postoperativ steigen kann, langfristig aber sinkt. Dies ist bisher nicht systematisch in Studien untersucht worden. Aus diesem Grund wird in einer retrospektiven Studie anhand von Krankenakten eines Patientenkollektivs mit Erkrankung der Makula untersucht, wie sich der IOD nach erfolgter Pars-plana-Vitrektomie in einem Zeitraum von 6-12 Monaten ändert und welche Einflussfaktoren hierbei eine Rolle spielen. 


\subsection{Anatomische Grundlagen}

Zum besseren Verständnis der Pathogenese von Makulaerkrankungen und der Regulation des intraokularen Drucks ist es wichtig, die Anatomie des Auges zu kennen.

Da als Hypothese der durchgeführten Studie eine Änderung des IOD postuliert wird, ist das Verständnis von Regulationsmechanismen des IOD und der Kammerwasserproduktion ebenfalls von Bedeutung. Im folgenden Abschnitt soll daher auf Anatomie und Physiologie des Auges mit besonderem Fokus auf die Makula und die Regulation des IOD eingegangen werden.

\subsubsection{Das Auge}

Das menschliche Auge ist das Sinnesorgan, welches die Wahrnehmung von visuellen Reizen wie Licht, Farbe und Bewegung ermöglicht. Es besteht aus dem Augapfel (Bulbus oculi) mit Sehnerv, dem Tränenapparat, den Augenlidern und den 6 äußeren Augenmuskeln. Topographisch liegt das Auge in der Orbita, einer knöchernen Höhle des Gesichtsschädels. Das Auge ist in der Lage, elektromagnetische Strahlung mit einer Wellenlänge im Bereich von 400-750 nm wahrzunehmen.

Der Augapfel gliedert sich anatomisch in einen vorderen und einen hinteren Bulbusabschnitt. Der vordere Teil wird von ventral in Konjunktiva (Bindehaut), Kornea (Hornhaut), Iris (Regenbogenhaut) mit darin befindlicher Pupille, Lens (Linse) mit daran befestigten Zonulafasern und dem Corpus ciliare (Strahlenkörper) mit vorderer Pars plicata und hinterer Pars plana eingeteilt. Dieser ist für die Änderung der Linsenkrümmung und damit für die Akkomodation (Naheinstellung des optischen Systems) verantwortlich.

Der hintere Teil des Bulbus stellt den sensorischen Anteil des Auges dar. Dieser lässt sich von dorsal in Sklera (Lederhaut), Uvea (Aderhaut) und Retina (Netzhaut) mit Pars caeca (aus lat. blind) und Pars optica (aus griech. Optikos, zum Sehen gehörend) gliedern. Das Corpus vitreum (Glaskörper) füllt den Raum zwischen Linse und Retina aus. 


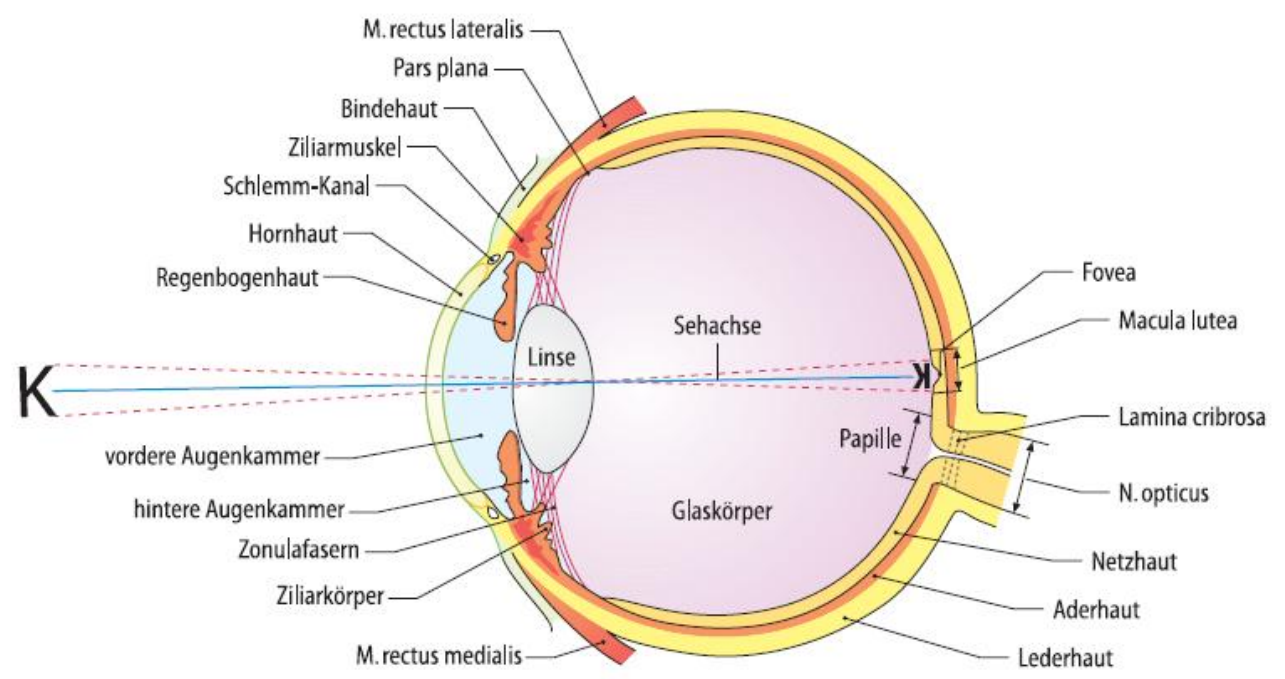

Abbildung 1: Übersicht Auge und seine Schichten (Grehn 2008) ${ }^{1}$

\subsubsection{Der Glaskörper}

Der Glaskörper (Corpus vitreum) ist ein durchsichtiges, nicht komplett homogenes Gel und füllt topographisch den Raum zwischen Linse und Netzhaut aus. Im antero-lateralen Bereich grenzt dieser an die Pars plana des Ziliarkörpers an, welcher als Zugangsweg für die Parsplana-Vitrektomie genutzt wird.

Die Zusammensetzung des Glaskörpers besteht zu $98 \%$ aus Wasser, $2 \%$ aus Hyaluronsäure, einem Mukopolysaccharid, das im nicht-pigmentierten Epithel der Pars plana des Ziliarkörpers produziert wird, und Kollagenfibrillen, vor allem vom Typ 2 (Foster 2008). Hyalozyten sind die im Glaskörper befindlichen Zellen, von denen vermutet wird, dass sie an der Bildung der Kollagenfibrillen des Typ 2 beteiligt sind (Kohno et al. 2009). Die Fibrillen lagern sich vor allem in der Peripherie an und sorgen für eine Verbindung zur Retina, die sogenannte Glaskörpergrenzmembran (Membrana hyaloidea). Zum Teil haftet diese auch an die innere Grenzmembran der Netzhaut (ILM) an, die von den Fußfortsätzen der Müller'schen Stützzellen der Netzhaut gebildet werden.

Als Überrest der Arteria hyaloidea aus der Embryonalzeit verläuft zwischen Retina und Linse mittig durch den Glaskörper der Canalis hyaloideus, der eine Verdichtungszone darstellt. Diese Arterie bildet sich 6 Wochen vor der Geburt zurück, woraufhin sich der Glaskörper anschließend mit Wasser füllt.

\footnotetext{
${ }^{1}$ Die Verwendung erfolgt mit freundlicher Genehmigung von Springer Nature (Abbildung 1.1, Seite 3)
} 
Der Glaskörper dient der Stabilisierung des Bulbus. Er nimmt circa zwei Drittel des Augengesamtvolumens ein. Das durchsichtige Gel des Glaskörpers wird von der Glaskörperrinde (Kortex) umgeben, die sich in einen vorderen und einen hinteren Teil gliedert. Diese Kortex besteht aus dicht gepackten Kollagenfibrillen und weist eine Dicke von etwa $100 \mu \mathrm{m}$ auf. Die vitreoretinale Verbindung wird durch den hinteren Teil der Kortex, den Ankerfibrillen des Glaskörpers, und der inneren Grenzmembran gebildet, die vor allem aus Kollagen 4 und Proteoglykanen besteht und 1-3 $\mu \mathrm{m}$ dick ist. Über den Bereich der Ora serata, einer gezackten Linie zwischen Pars caeca und optica der Retina, und um den Sehnervenkopf besteht eine feste Verbindung zwischen Glaskörper und Retina. Leichte physiologische Adhärenzen finden sich auch im Bereich der Makula. Die übrige Fläche der Netzhaut ist nicht mit der Glaskörpergrenzmembran verbunden. Pathologische Anhaftungen, vor allem in der Peripherie der Netzhaut, können im Rahmen von altersbedingten Umbauvorgängen des Glaskörpers mit Abhebung der Glaskörpergrenzmembran zu Rissen und Löchern in der Netzhaut und damit zur Netzhautablösung führen.

\subsubsection{Die Retina}

Die Retina ist die innerste 200-500 $\mu \mathrm{m}$ dicke durchsichtige Schicht des hinteren Abschnitts des Bulbus oculi. Sie wird in eine ventrale Pars caeca und eine dorsale Pars optica eingeteilt, die durch die Ora serata voneinander getrennt werden. Die Pars optica stellt den sensorischen Anteil des Auges dar und enthält die Sinneszellen, die für die Wahrnehmung von Licht verantwortlich sind. Embryologisch stammt das Gewebe der Retina vom Neuroektoderm ab. In der Pars optica sind sowohl der Sehnervenkopf als auch die Makula mit der Fovea centralis lokalisiert. Histologisch lässt sich die Retina in zehn verschiedene Schichten einteilen: 


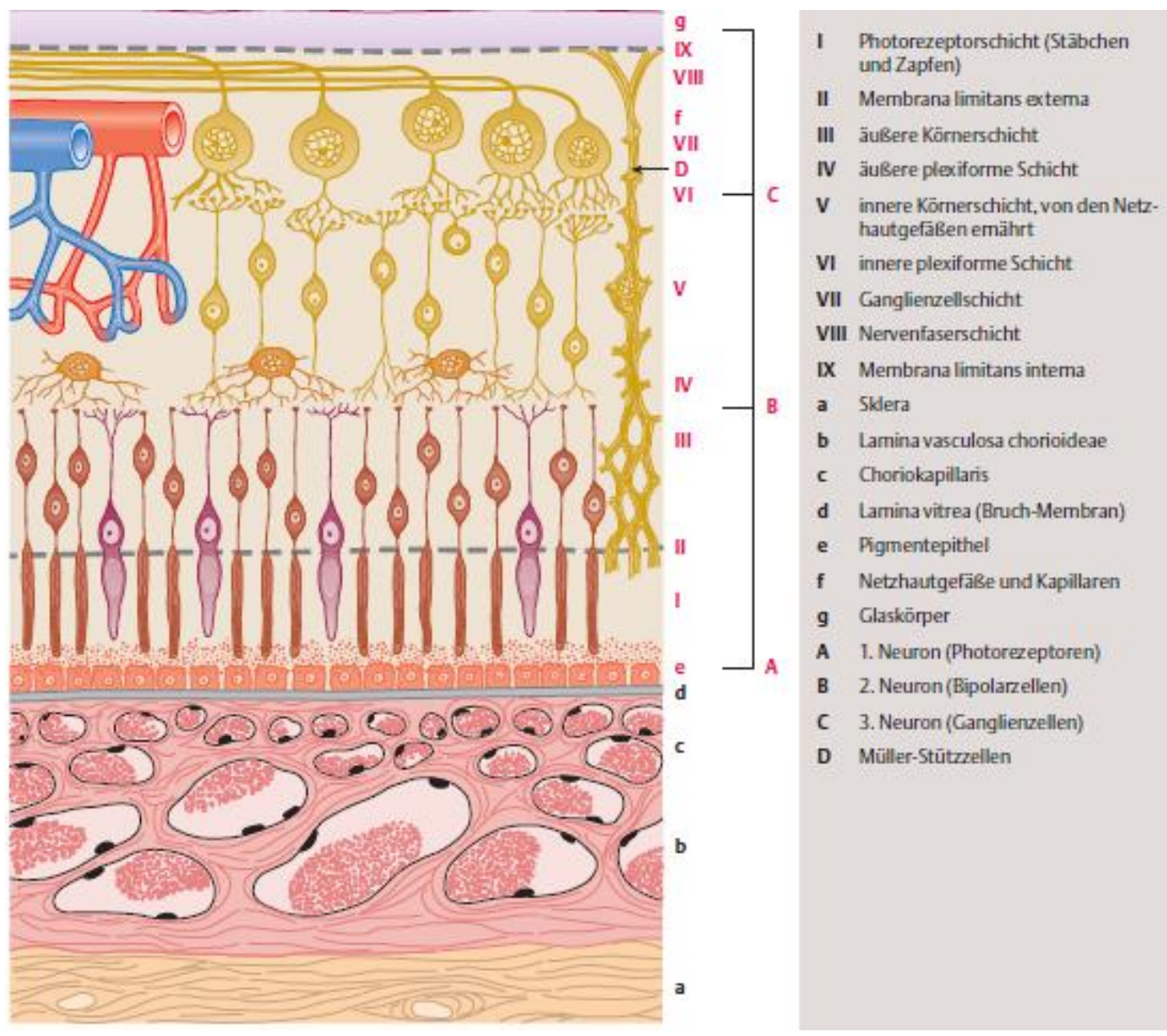

Abbildung 2: Schematischer Aufbau der Retina (Sachsenweger 2003) ${ }^{2}$

1) Stratum pigmentosum (Pigmentepithelschicht)

2) Stratum neuroepitheliale (Photorezeptorenschicht: Stäbchen und Zapfen)

3) Stratum limitans externum (Äußere Grenzschicht)

4) Stratum nucleare externum (Äußere Körnerschicht)

5) Stratum plexiforme externum (Äußere plexiforme Schicht)

6) Stratum nucleare internum (Innere Körnerschicht)

7) Stratum plexiforme internum (Innere plexiforme Schicht)

8) Stratum ganglionicum (Ganglienzellschicht)

9) Stratum neurofibrarum (Nervenfaserschicht)

10) Stratum limitans internum (Innere Grenzschicht, ILM)

Das äußerste, am weitesten distal gelegene Stratum pigmentosum, besteht aus retinalem Pigmentepithel. Diese Zellen sind fest auf der Bruch-Membran verankert und über tight

\footnotetext{
${ }^{2}$ Die Verwendung erfolgt mit freundlicher Genehmigung des Thieme-Verlags (Abbildung 14.1, Seite 249)
} 
junctions untereinander verbunden. Das Stratum pigmentosum bildet gemeinsam mit dem Endothel der versorgenden Gefäße die Blut-Retina-Schranke. Das Pigmentepithel dient dem Sauerstoffaustausch zwischen der Choriokapillaris und den Photorezeptorzellen. Zudem enthält es das Pigment Melanin, welches das Auge vor Streulicht schützt.

Die anderen neun Schichten der Retina gehören zur Pars nervosa, die die Photorezeptorzellen, die Stäbchen und Zapfen (1. Neuron), enthält. Die Stäbchen (etwa 120 Millionen) sind außerhalb der Makula angesiedelt und aufgrund ihrer starken Lichtempfindlichkeit für das Dämmerungssehen bzw. generell für die Wahrnehmung von Helligkeitsunterschieden zuständig. Die Zapfen (etwa 6 Millionen) liegen hauptsächlich in der Makula und sind für das Sehen bei Tageslicht und die Wahrnehmung von Farben verantwortlich. Sie haben eine eher geringe Lichtempfindlichkeit.

Das zweite Neuron bilden die Bipolarzellen in der inneren Körnerschicht. Sie nehmen meist elektrochemische Reize mehrerer Photorezeptorzellen auf. Nur in der Makula, am Ort des schärfsten Sehens, werden die Informationen zwischen erstem und zweitem Neuron eins-zueins verschaltet. Anschließend leiten sie das Signal z. T. über amakrine Zellen an die Ganglienzellen (3. Neuron) in der Ganglienzellschicht weiter.

Die Müller-Zellen, eine Sonderform der Astroglia, durchziehen die Retina von der äußeren zur inneren Grenzschicht und spielen eine wichtige Rolle in der Regulation des extrazellulären Milieus der Netzhaut, um eine optimale Funktion der Neurone zu gewährleisten. Alle Axone der Ganglienzellen vereinen sich anschließend in der Papille, dem Sehnervenkopf, und bilden dort den Nervus opticus, der die Lichtsignale in Richtung Zwischenhirn weiterleitet.

Die innerste Schicht der Netzhaut, die dem Glaskörper direkt anliegt, ist die innere Grenzmembran (ILM), die aus den Fortsätzen der Müller-Zellen gebildet wird und deren Basalmembran darstellt.

\subsubsection{Die Makula}

Die Makula ist ein ovaler, gefäßloser Fleck am hinteren Pol der Retina mit einem Durchmesser von etwa $3 \mathrm{~mm}$. Topographisch befindet sie sich in der Mitte der Netzhaut und enthält im Zentrum eine trichterförmige Vertiefung, die sogenannte Fovea centralis, die Stelle der höchsten Sehschärfe. Wiederum im Zentrum der Fovea centralis befindet sich die Foveola, in 
der die Zapfen nur von Ausläufern der Müllerzellen bedeckt sind, wodurch das Licht nicht durch darüberliegende Zellen gestreut werden kann.

In der Fovea centralis ist die Dichte an Zapfen, die eins-zu-eins mit den Bipolarzellen verschaltet werden, am höchsten. Ein Drittel aller Ganglienzellaxone kommen aus der Makula, was verdeutlicht, wie wichtig die Makula für die Sehschärfe ist. Sowohl das optische Auflösungsvermögen als auch die Rezeptorzelldichte nehmen in Richtung Retinaperipherie mit Abstand zur Makula exponentiell ab.

\subsection{Der intraokulare Druck}

\subsubsection{Definition}

Der intraokulare Druck (IOD) ist der Druck, der auf der Augeninnenwand lastet. Dieser wird in der Einheit Millimeter Quecksilbersäule (mmHg) angegeben. In gesunden Augen generiert der Kammerwasserfluss gegen Widerstand einen physiologischen IOD von etwa $15 \mathrm{mmHg}$ (Maximum 21, Minimum 10 mmHg) (True Gabelt und Kaufmann 2011). Der IOD dient nicht nur der Aufrechterhaltung der Form des Augapfels, sondern auch der Ernährung von avaskulären Geweben des Auges. Das Kammerwasser ist ein Blutanalogon dieser avaskulären Gewebe, stellt Nährstoffe zur Verfügung, entfernt Stoffwechselabbauprodukte, transportiert Neurotransmitter, stabilisiert die Struktur des Auges und hält die Homöostase der Gewebe des Auges aufrecht (Goel et al. 2010). Der IOD wird durch Verformung des Augapfels, meist transkorneal, gemessen. Goldstandard ist die Applanationstonometrie nach Goldmann (Goldmann und Schmidt 1957), bei der die aufgewendete Kraft zur Abplattung (Applanation) einer definierten Fläche der Kornea gemessen wird. Die Messung wird unter anderem von der Dicke der Kornea oder dem Oberflächenradius beeinflusst (Shah 2000).

\subsubsection{Regulation des intraokularen Drucks}

Der IOD wird zum einen über die Menge des produzierten Kammerwassers reguliert, zum anderen wird er vom Abflusswiderstand am Trabekelmaschenwerk (TMW) (Horio und Horiguchi 2004; Kelly und Wendel 1991) und am uveoskleralen Abflusssystem sowie vom Gegendruck in den episkleralen Venen beeinflusst. Bei Vorliegen eines IOD-Anstiegs oder Abfalls müssen also Veränderungen eines oder mehrerer der genannten physiologischen Regulationsmechanismen vorliegen. 


\subsubsection{Die Kammerwasserbildung}

Der Ziliarkörper ist für die Bildung des Kammerwassers zuständig. Er gliedert sich in eine vordere Pars plicata, in der die Ziliarzotten liegen, und eine hintere Pars plana. Das Kammerwasser wird kontinuierlich durch Ultrafiltration des zirkulierenden Blutflusses in der Pars plicata des Ziliarkörpers produziert, wobei Wasser und gelöste Substanzen bedingt durch einen hydrostatischen Druckgradienten durchs Kapillarendothel gepresst werden. Anschließend gelangen Anionen, Kationen und andere Substanzen durch das nichtpigmentierte Ziliarepithel, bilden dort einen Gradienten und ziehen somit Wasser in den interzellulären Zwischenraum. Dieser ist apikal über tight junctions verschlossen und basal geöffnet. Über die basale Öffnung des interzellulären Zwischenraums gelangt das Kammerwasser in die hintere Augenkammer. Von dort gelangt es durch die Pupille in die vordere Augenkammer.

\subsubsection{Trabekulärer Abfluss}

Das Kammerwasser fließt zum größten Teil über das Trabekelmaschenwerk (TMW), einer siebartigen Öffnung, die im Winkel zwischen Iris und Kornea (Angulus iridocornealis) gelegen ist, in den Schlemm-Kanal. Die innerste Schicht, das juxtacanaliculäre Endothel des Trabekelmaschenwerks, ist in der Regel die Struktur, die den größten Flusswiderstand erzeugt und daher hauptsächlich den Kammerwasserabfluss reguliert (Johnson 2006; Ethier 2002). Eine Minderung des Abflusswiderstandes kann durch Kontraktion des Ziliarmuskels erreicht werden, indem in das TMW inserierende Fasern Zug auf das TMW ausüben und seine Mikrostruktur verändern. Im Alter steigt der Abflusswiderstand, während die Kammerwasserproduktion eher sinkt. 


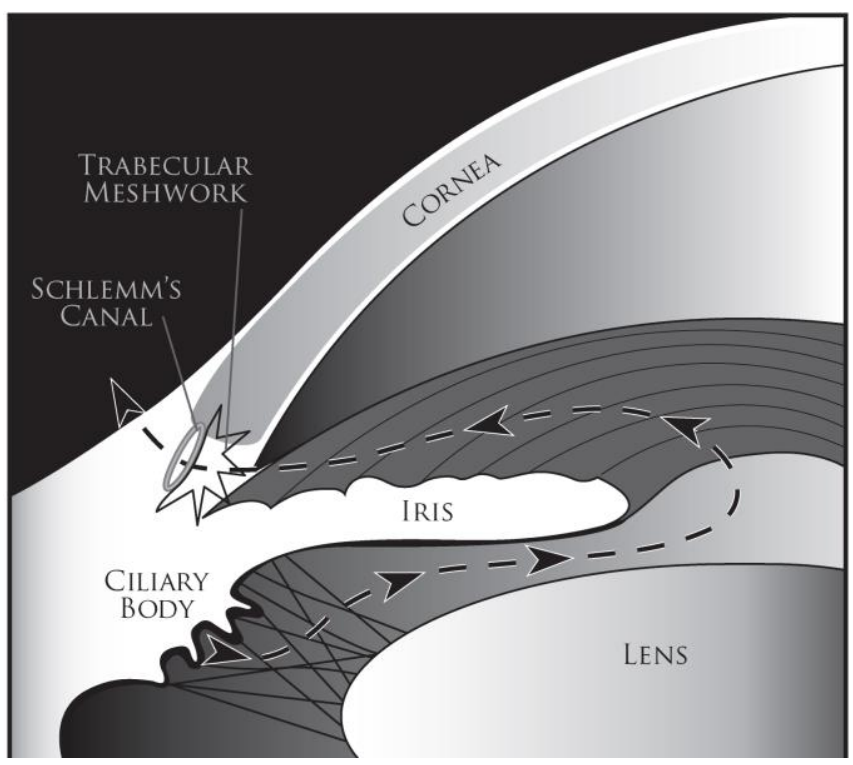

Abbildung 3: Schematische Darstellung des trabekulären Abflusses (Goel et al. 2010) ${ }^{3}$

Aus dem Schlemm-Kanal gelangt das Kammerwasser über Kollektorkanäle in Kammerwasservenen und schließlich in den episkleralen Venenplexus. Bei gesunden Menschen liegt der episklerale Venendruck bei Werten zwischen 7 und $14 \mathrm{mmHg}$ (meist bei 9-10 mmHg), korreliert mit dem IOD und bleibt über den Tag relativ konstant. Ein Anstieg des episkleralen Venendrucks um 0,8 mmHg führt zu einem IOD-Anstieg von $1 \mathrm{mmHg}$ (Toris 2015). Die zirkadianen Tagesschwankungen des IOD zeigen Parallelen zu den Schwankungen des episkleralen Venendrucks (Blondeau et al. 2001).

\subsubsection{Der uveosklerale Abfluss}

Der uveosklerale Abfluss stellt den eher unkonventionellen, da weniger vom IOD abhängigen Abfluss dar. Dieser verläuft von der Iris durch das uveale Maschenwerk durch die Vorderseite des Ziliarmuskels. Anschließend verläuft dieser durch das Verbindungsgewebe zwischen den Muskelfasern und dem Subarachnoidalraum durch die Sklera in Lymphgefäße, welche außerhalb des Auges (Orbita) liegen. Der uveosklerale Abflussbereich ist eine Erweiterung des Ziliarmuskels aus großen überlappenden Löchern $(25-75 \mu \mathrm{m})$ und abgeflachten Bögen, die in verschiedenen Ebenen miteinander verbunden sind. Man vermutet, dass der uveosklerale Ausfluss durch Druckgradienten über die Uvea, Bewegungen des Ziliarmuskels und zytoskelettale Veränderungen der Extrazellulärmatrix des filternden Gewebes beeinflusst wird.

\footnotetext{
${ }^{3}$ Creative Commons Attribution Non-Commercial 3.0 Lizenz
} 


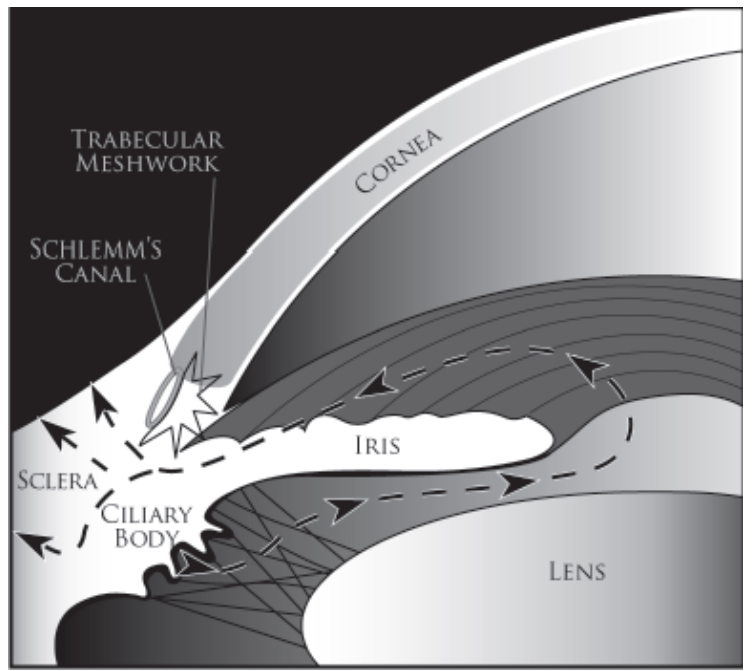

Abbildung 4: Schematische Darstellung des uveoskleralen Abflusses (Goel et al. 2010) ${ }^{4}$

Die maximale, vor allem nachts relevante Abflussrate über den uveoskleralen Abfluss beträgt bei jungen Leuten 35-55 \% des Kammerwasserflusses (True Gabelt und Kaufmann 2011) und nimmt mit dem Alter signifikant ab. Augen von Patienten im Alter von 20-30 Jahren hatten in einer Studie von Toris et al. im Durchschnitt einen uveosklerale Abflussrate von 54 \%, Patienten über 60 Jahren eine von $46 \%$ (Toris et al. 1999).

\subsubsection{Einflussfaktoren auf den IOD}

Die Produktionsrate des Kammerwassers ist nahezu konstant, nimmt jedoch mit steigendem Alter ab. Die Bildungsrate in Augen junger Patienten liegt bei 2,7 $\mu 1 / \mathrm{min}$, die bei Patienten über 80 Jahren bei $2,2 \mu \mathrm{l} / \mathrm{min}$ (Brubaker 1991). Der IOD unterliegt zirkadianen Schwankungen: In den frühen Morgenstunden ist er am höchsten, in der Nacht erreicht er seinen Tiefpunkt mit nur $43 \%$ des morgendlichen Drucks mit einer Flussrate von 3,1 $\mu 1 / \mathrm{min}$ morgens und 1,6 $\mu \mathrm{l} / \mathrm{min}$ nachts (Reiss et al. 1984). Bei vielen Glaukompatienten findet man eine vergrößerte Amplitude der tageszeitlichen Druckschwankungen und nächtliche Druckspitzen sind häufiger. Verantwortlich für die tageszeitlichen Schwankungen sind zum Beispiel hormonelle Regulationsvorgänge (Viggiano et al. 1994). Durch die Änderung der Körperposition (aufrecht vs. liegend), sowie tageszeitliche Schwankungen kann der IOD ebenfalls beeinflusst werden. Eine Studie an gesunden Patientenaugen konnte nachts eine Verminderung des Kammerwasserflusses und -abflusses sowie auch des uveoskleralen Abflusses feststellen. Der uveosklerale Abfluss hat nachts einen geringeren Anteil an der Drainage als tagsüber (Liu et al. 2011). Eine mögliche Erklärung hierfür ist eine verminderte Kammer-

\footnotetext{
${ }^{4}$ Creative Commons Attribution Non-Commercial 3.0 Lizenz
} 
wasserproduktion, welche dazu führt, dass weniger Flüssigkeit durch die uveoskleralen Gewebe abläuft. Nau und Kollegen (2013) konnten an gesunden Augen älterer Patienten eine ca. $50 \%$ reduzierte Kammerwasserflussrate nachts im Vergleich zu tags messen, jedoch keinen IOD-Unterschied. Dies erklärten sich die Autoren ebenfalls durch einen stark verminderten uveoskleralen Abfluss und verminderten Abflusswiderstand (Nau et al. 2013). Photo- oder Kryokoagulationsnarben von ophthalmochirurgischen Eingriffen im Bereich der Pars plana des Ziliarkörpers können, wahrscheinlich durch eine Verbesserung des uveoskleralen Abflusses, zu einer milden IOD-Senkung führen.

Der Pathomechanismus eines pathologisch erhöhten IOD wurde in zahlreichen Studien untersucht, dieser ist jedoch weiterhin unklar. Das TMW spielt wahrscheinlich die wichtigste Rolle bei der Entstehung von IOD-Anstiegen. Man fand heraus, dass das TMW ein den IOD und Kammerabfluss regulierendes Gewebe ist, welches eine essentielle Schlüsselrolle in der Pathogenese der Druckerhöhung spielen kann. Durch Änderungen in genanntem System entsteht ein erhöhter Widerstand im Ausflusssystem. Der Widerstand des Kammerwasserabflusses wird zu $75 \%$ im Gewebe zwischen vorderer Augenkammer und dem Lumen des Schlemm-Kanals geregelt. Das TMW stellt einen biologischen Filter im Auge dar, der vom Kammerwasser durchquert werden muss, um in den Schlemm-Kanal zu gelangen. LutjenDrecoll et al. fanden heraus, dass die Zellen des TMWs des Auges wesentlich für das Aufrechterhalten der Homöostase des Kammerwasserabflusses sind und somit auch bei Störung des Systems, wie vermehrte Ablagerungen extrazellulärer Matrixproteine, eine zentrale Rolle in der IOD-Erhöhung und der konsekutiven Glaukomentstehung spielen (Lütjen-Drecoll 1999).

In einer Studie mit Patienten mit Offenwinkelglaukom zeigte sich, dass es einen statistisch signifikanten Zusammenhang zwischen oxidativem Stress an Trabekelmaschenwerkszellen durch das Sauerstoffradikal 8-OH-dG und dem intraokularen Druck gibt. Hierbei standen die physiologischen Tagesfluktuationen des IOD in keinem Zusammenhang mit oxidativem Stress, wohingegen die Maximal- und Minimaldrücke jeweils mit dem oxidativen Stress im TMW korrelierten (Saccà et al. 2005).

Studien vermuten, dass auch Glukokortikoide eine wesentliche Rolle in der physiologischen Regulation des Kammerwasserabflusses und des IOD spielen. Man fand in den Zellen des Kammerwasserabflusses Glukokortikoidrezeptoren. Die Gabe von Steroiden, sowohl topisch als auch systemisch, vermindert die Clearance von extrazellulärer Matrix (komplexe Kohlenhydrate, Hyaluronsäure, Proteine und Kollagensynthese) aus dem TMW sowie deren Ver- 
teilung in den Zellen und im Gewebe des Kammerwasserabflusssystems. Hierüber kann es zu einer Erhöhung des intraokularen Drucks kommen. Dies wird als Steroidresponse bezeichnet. Glaukompatienten haben im Vergleich $\mathrm{zu}$ gesunden Individuen ein höheres Plasmakortisonlevel sowie ein gesteigertes Ansprechen auf Kortikoide (True Gabelt und Kaufmann 2011).

Auch Myocilin, ein Glykoprotein, welches in großen Mengen im TMW, der Sklera, dem Ziliarkörper und der Iris sezerniert wird, wird in der Pathogenese des IOD-Anstiegs diskutiert. Bei einigen Patienten mit primären Offenwinkelglaukomen fand man eine Mutation des Gens für Myocilin. Auch im Kammerwinkel ließ sich Myocilin im kribiformen Teil des Trabekelwerks mit spezifischer Bindung an die HEPII-Domäne von Fibronektin nachweisen; eine Myocilin-Mutation spielt wahrscheinlich eher bei den selteneren hereditären Glaukomformen eine Rolle (Ohlmann und Tamm 2002).

\subsection{Erkrankungen der Makula}

\subsubsection{Makulaforamen}

Das Makulaforamen (MF) stellt vor allem bei älteren Menschen eine häufige Ursache für einen Verlust des zentralen, scharfen Sehens dar. Dieses Foramen ist in seiner vollen Ausprägung ein Defekt der gesamten Netzhautdicke, der sich in der Fovea, dem Punkt des schärfsten Sehens, entwickelt.

Aber nicht nur das Alter, sondern auch das weibliche Geschlecht und eventuell erhöhte Fibrinogen-Konzentrationen sind Risikofaktoren ein MF zu entwickeln (The Eye Disease CaseControl Study Group 1994). Frauen sind zwei- bis dreimal häufiger betroffen als Männer (Gass 1988). Bei einem erkrankten Auge besteht die Gefahr, dass in 7-15\% der Fälle am anderen Auge ebenfalls ein Makulaforamen entsteht. In einer Studie wurde eine beidseitige Erkrankung bei einem Viertel der Patienten beschrieben (Kelly und Wendel 1991). Die Prävalenz des Makulaforamens wird in der Literatur mit 2-33/10.000 bei Patienten älter als 55 Jahren angegeben (Freeman 1993; Mitchell et al. 1997). Die Inzidenz liegt durchschnittlich bei 7,8/100.000/Jahr, wobei die der Frauen mit 11,6 höher als die der Männer mit 5,0 pro 100.000 liegt (McCannel et al. 2009).

1869 beschrieb Knapp erstmals das Krankheitsbild des Makulaloches als Folge eines intraokularen Traumas. Die Bezeichnung Makulaforamen bezieht sich meistens auf die häufigste 
Form, das idiopathische MF, dessen Ursache unbekannt ist, und das vor allem mit steigendem Alter auftritt (Dithmar 2005). Das Makulaforamen kann aber auch nicht-idiopathischer Genese sein. Dies kann z. B. durch starke Myopie oder ein okulares Trauma (Kontusionstrauma) verursacht werden (Ho et al. 1998; Parravano et al. 2015). Weitere Risikofaktoren für das Auftreten eines Makulaforamens können Solarretinopathien und mit Laserkoagulation behandelte Augen sein. Auch bei bestehender epiretinaler Gliose ist das Risiko für das Auftreten eines MF erhöht.

Zur Pathogenese gibt es diverse Theorien: Von posttraumatischen Ursachen (Noyes 1871) über zystische Degeneration bis hin zu vaskulären Theorien mit Angiospasmen (Schweinitz 1904). Die genaue Pathogenese ist bis heute nicht vollständig verstanden, wobei Gass 1988 mit seiner detaillierten Forschungsarbeit über die Pathogenese des Makulaforamens die Wissenschaft einen großen Schritt voranbrachte (Gass 1988; Smiddy und Flynn 2004). Nach Überarbeitung seiner Arbeit publizierte Gass diese 1995 erneut, sie wurde dann allgemein anerkannt. Er beschrieb hierin auf der Basis seiner biomikroskopischen Beobachtungen vier verschiedene Stadien des Makulaforamens (Gass 1995):

Stadium IA ist ein funduskopisch sichtbarer zentraler, etwa 50-100 $\mu \mathrm{m}$ umschriebener gelber Fleck. In diesem noch sehr frühen Stadium werden die äußersten Foveaschichten durch vitreofoveale tangentiale Traktion angehoben. Dieser Zug kommt durch Schrumpfung der vor der Makula gelegenen Glaskörperrinde zustande. Die Patienten können außer leichter Metamorphopsie (Verzerrtsehen) symptomlos sein, der Visus bleibt meistens unbeeinträchtigt.

Stadium IB ist ein funduskopisch sichtbares Fortschreiten des „gelben Flecks“ aus Stadium IA zu einem ,gelben Ring“, der einen Durchmesser von etwa 200-300 $\mu$ m zeigt. Dies kommt durch die vermehrten vitreofovealen Kräfte und dadurch bedingte fortschreitende Abhebung der Fovea zustande und führt zum Einriss der Photorezeptorenschicht. Die innersten Retinasegmente bleiben hierbei intakt. Auch in diesem Stadium klagen die Patienten über geringgradige Metamorphopsie sowie z. T. leichte Einschränkung (Verschwommen sehen) beim Lesen.

Stadium II zeigt funduskopisch den Übergang in ein durchgreifendes Makulaforamen. Dies kann einen Durchmesser bis $<400 \mu \mathrm{m}$ aufweisen. Es sind jetzt aufgrund der tangentialen 
Traktion auch die inneren Retinaschichten eingerissen. Die Patienten klagen über vermehrte Metamorphopsie und fortschreitenden Visusverlust. Die Lesefähigkeit bleibt noch erhalten.

Stadium III unterscheidet sich von Stadium 2 in seinem Durchmesser von $>400 \mu \mathrm{m}$. Zudem lässt sich bei $75 \%$ der Patienten ein verdichtetes Gewebe finden, was stark reflektierend ist. Diese Verdichtung entspricht der abgehobenen Glaskörpergrenzmembran über dem Makulaloch. Die Glaskörperrinde über der Fovea löst sich ab, wohingegen der Glaskörper kreisrund um das Foramen noch anliegt. Gass bezeichnete dies als Pseudo-Operkulum. Der Visus kann in diesem Stadium deutlich eingeschränkt sein.

Stadium IV ist eine vollständige hintere Glaskörperabhebung. Hierbei ist die Größe des Makulaloches nicht von Bedeutung. Durch einen subretinal vorhandenen Flüssigkeitssaum kann es zur Atrophie des retinalen Pigmentepithels (RPE) (Casuso et al. 2001) und der Photorezeptoren kommen. Konsekutiv klagen die Patienten über starken Visusverlust und zum Teil das Auftauchen von Gesichtsfeldausfällen (Skotomen).

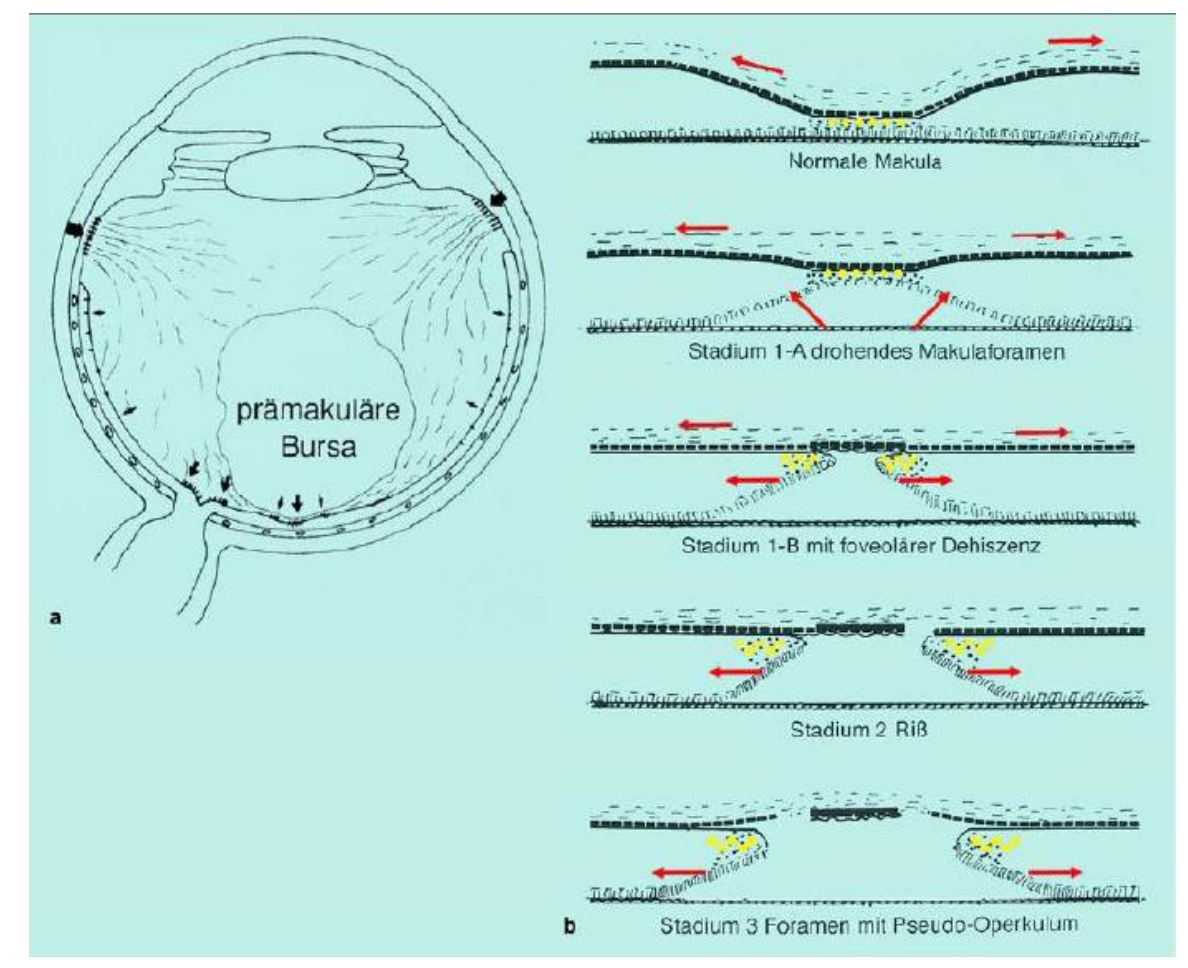

Abbildung 5: Pathomechanismus und Stadieneinteilung des Makulaforamens nach Gass (Dithmar 2005) $)^{5}$

\footnotetext{
${ }^{5}$ Die Verwendung erfolgt mit freundlicher Genehmigung des Springer-Verlags
} 
Mit der Einführung der OCT können die genauen Wechselwirkungen zwischen Glaskörper und Retina besser erforscht und verstanden werden. Die OCT stellt ein nicht-invasives bildgebendes Verfahren dar, das die anatomischen Begebenheiten in deutlich höherer Auflösung und als optisches Schnittbild darstellt, während biomikroskopische Bilder nur die Aufsicht auf das Gewebe ermöglicht (Altaweel und Ip 2003). Im Gegensatz zu Gass' Erkenntnissen zeigt sich in der OCT eine perifoveoläre hintere Glaskörperabhebung bereits im Stadium 1 und 2. Bis Stadium 2 liegt eine anterior-posteriore Traktion und nicht wie bei Gass beschrieben ein tangentialer Zug vor. Erst ab Stadium 3 entstehen nach Retinaeinriss im vorherigen Stadium tangentiale Kräfte, die durch Einwanderung von RPE-Zellen mit konsekutiver epiretinaler Zellproliferation verursacht werden. In einer Studie wurde beobachtet, dass eine Degeneration der obersten Retinaschicht eine Prädisposition für die Bildung eines MF darstellt, da so möglicherweise zufällige Zugkräfte, die senkrecht zur Retinaoberfläche ausgerichtet sind, durch fokale Proliferation von Müller-Zellen die Bildung des Loches begünstigen (Smiddy und Flynn 2004). Für die Entstehung eines Makulaforamens spielen 3 Elemente eine wesentliche Rolle: fokale vitreofoveale Adhäsion, sowie tangentiale und anterior-posteriore Traktion (Altaweel und Ip 2003). Gass' Einteilung der Pathomechanismen hat seit Einführung des OCT nur noch eingeschränkte Gültigkeit, seine Stadieneinteilung wird jedoch weiterhin international angewandt.

Makulaforamina heilen nur sehr selten spontan und bedürfen daher in der Regel einer Therapie. Den betroffenen Patienten fällt auf, dass sie oft kleine Gegenstände und Geschriebenes nicht mehr erkennen können, vor allem beim Verschließen des gesunden Auges. Typisch ist eine langsam progrediente Verschlechterung über einen Zeitraum von mehreren Monaten, bis sich sowohl die Lochgröße, als auch der Visus in Stadium 3 und 4 stabilisieren (Casuso et al. 2001; Hikichi et al. 1995). In Stadium 1 und z. T. auch in Stadium 2 gibt es Angaben in der Literatur, dass sich die Foramina spontan zurückbilden können. Im Stadium 2 muss in $84 \%$ der Fälle mit einer Progression in ein durchgreifendes Makulaforamen gerechnet werden, in Stadium 1 nur in $33 \%$ der Fälle (Hikichi et al. 1995). Diagnostiziert wird das Makulaforamen über optische Kohärenztomographie (OCT) oder Augenhintergrundspiegelung, die sogenannte Funduskopie.

Die Therapie muss von Stadium zu Stadium unterschiedlich angepasst werden. Therapie der Wahl ist aber die Vitrektomie mit kombiniertem ILM-Peeling, das heißt die Entfernung der inneren Grenzmembran mit anschließender Luft- oder Gasfüllung. Auf dieses Operationsverfahren soll im Kapitel 1.4 genauer eingegangen werden. 


\subsubsection{Epiretinale Gliose}

Die epiretinale Gliose (EG) oder auch Makulapucker ist eine fibrozelluläre Membran, die sich auf der Innenoberfläche der Netzhaut, meist im Bereich der Makula bildet. Ursächlich hierfür sind die Proliferation von Gliazellen (Müller-Zellen und Astrogliazellen) und die Produktion eines fibrösen Gewebes durch myofibroblastenartige Zellen auf der retinalen Oberfläche.

Die EG wurde 1865 erstmals in „Studien über die normale und die pathologische Anatomie des Auges“"von Iwanoff beschrieben (Iwanoff 1865) und betrifft in der Regel Patienten ab 50 Jahren und älter.

In verschiedenen Studien wurden zwei Haupttypen der epiretinalen Gliose definiert: Eine weniger gravierende Form, genannt Zellophanmakulopathie (cellophane macular reflex, $\mathrm{CMR}$ ) und eine gravierendere Form, genannt präretinale Makulafibrose (preretinal macular fibrosis, PMF) (Fraser-Bell et al. 2003; Mitchell et al. 1997).

Die CMR ist eine dünne, transparente, im Funduskop glänzende Membran, die die frühe Form der EG darstellt. Die Patienten sind meist asymptomatisch. Die fortgeschrittene Form PMF kann hingegen zu Metamorphopsie und Visusverlust durch tangentialen Zug an der Retina im Bereich der Makula führen (Bu et al. 2014; Foos 1977). Diese Metamorphopsie entsteht durch Kontraktion der Myofibroblasten, wodurch sich eine Falte (englisch: pucker) bildet. In einer Studie zeigte sich, dass die CMR in die PMF übergehen kann (Fraser-Bell et al. 2003).

Die Ursache der EG ist meist idiopathisch, kann aber auch durch retinale Gefäßverschlüsse, okulares Trauma, intraokulare Chirurgie, Uveitis oder hintere Glaskörperabhebung bedingt sein. Die Häufigkeit der EG korreliert mit steigendem Lebensalter, wie die „Blue-MountainsEye-Studie“ zeigt. Patienten dieser Studie wiesen unter 60 Jahren eine Prävalenz von 1,9\% auf. Zwischen 60 und 69 Jahren lag diese bei 7,2 \%, zwischen 70 und 79 Jahren bei 11,6\% und bei über 80-Jährigen bei 9,3\% (Mitchell et al. 1997). Die durchschnittliche Prävalenz lag bei 7,7\% für Frauen und $6 \%$ für Männer (Mitchell et al. 1997). Klein et al. wiesen alle Altersstufen eingeschlossen, eine Prävalenz von 11,8\% nach (Klein et al. 1994). In der „Melbourne Collaborative Cohort Study“ zeigt sich eine Prävalenz von 8,9\%. Hierbei ergab sich ein doppelt so hohes Auftreten bei Südeuropäern wie bei Nordeuropäern (Aung et al. 2013). Das Risiko, beidseits eine idiopathische EG zu entwickeln, beschrieben Hikichi und Trempe mit $21 \%$ (Hikichi und Trempe 1995).

Aber nicht nur das Alter, sondern auch metabolische Erkrankungen wie Diabetes, Hypercholesterinämie und Gefäßverschlüsse sowie Lebensstil, Bildungsstand und Ametropie 
beeinflussen die Häufigkeit der idiopathischen EG. Patienten in höherem Alter mit Retinaerkrankungen (z. B. Retina-Ablösung oder retinale Gefäßerkrankungen), diabetischer Retinopathie oder nach Kataraktextraktion zeigen eine höhere Prävalenz für EG als Patienten ohne diese Einflussfaktoren. Patienten mit diabetischer Retinopathie haben ein höheres Risiko, im Alter eine EG zu entwickeln, als Patienten nach Kataraktchirurgie (Klein et al. 1994). Eine weitere Studie der „Blue-Mountains-Eye“-Studiengruppe zeigt eine 5-Jahres-Inzidenz von 5,3\% der EG bei älteren Menschen (Fraser-Bell et al. 2003).

Der fibrotische Umbauprozess auf der Netzhautoberfläche wurde in vielen Studien erforscht. Eine genaue Ursache und Möglichkeiten einer frühen medikamentösen Behandlung der Fibrose sind bisher nicht gefunden. Genetische Aspekte und Lebensstil werden als Einflussfaktoren vermutet. Eine Schlüsselrolle in der Entwicklung der idiopathischen epiretinalen Gliose spielt die Transdifferenzierung vermutlich von Hyalozyten zu kontraktilen Myofibroblasten. Hierbei sind auch das Pigmentepithel und retinale Gliazellen, sowie Transforming growth factor beta (TGF-ß) als Signalprotein bedeutsam (Parapuram et al. 2009). Hyalozyten sind im Glaskörper befindliche Zellen myeloiden Ursprungs und gehören zum Monozyten-/Makrophagensystem. Studien zeigten, dass Hyalozyten die Fähigkeit haben zu proliferieren und sich zu myofibroblastenähnlichen Zellen zu differenzieren, die wesentlich zur Kollagen- und Aktinproduktion beitragen (Vagaja et al. 2012). Unter dem Einfluss von TGF- $\beta$ bilden Hyalozyten vermehrt hyperkontratiles Kollagen und alpha-SMA, ein glattmuskuläres Aktinfilament (Kohno et al. 2009). Dieses Aktin ist ein wichtiger Bestandteil, der zusammen mit dem gebildeten Kollagen für die Kontraktilität der EG sorgt, die dann zum Makulapucker führen kann. Auch Gliazellen (Astrozyten und Müller-Zellen) tragen zur Kollagenproduktion, v. a. von Kollagen 6 bei (Kritzenberger et al. 2011). Dass Gliazellen wesentlich an der Bildung der EG beteiligt sind, wurde auch in einer Studie bestätigt (Snead et al. 2004).

Foos beschrieb das vermehrte Auftreten epiretinaler Gliosen nach hinterer Glaskörperabhebung. Seine Theorie war, dass durch die hintere Glaskörperabhebung Mikroschäden an der inneren Grenzmembran entstehen, wodurch die Einwanderung retinaler Gliazellen und deren Proliferation und Entdifferenzierung zu Myofibroblasten ermöglicht wird (Foos 1977). Kataraktchirurgie fördert zudem die Entstehung einer hinteren Glaskörperabhebung, wodurch das Risiko, nach diesem Eingriff eine EG zu entwickeln, nochmals erhöht wird (Klein et al. 1994; Mitchell et al. 1997), was auch in einer Studie in Lateinamerika bestätigt wurde (Fraser-Bell et al. 2004). 
Bei Patienten mit Kern- oder Rindenkatarakt zeigte sich eine geringere Häufigkeit epiretinaler Gliosen. Ein weiterer protektiver Faktor bestand darin, schon früh an einer altersbedingten Makulopathie gelitten zu haben. Die Gründe hierfür sind aber bisher nicht bekannt (Klein et al. 1994).

\subsubsection{Vitreomakuläres Traktionssyndrom}

Das vitreomakuläre Traktionssyndrom (VMT) ist eine relativ seltene Sonderform der epiretinalen Gliose. Durch inkomplette hintere Glaskörperabhebung kommt es zu einer persistierenden Verbindung zwischen Makula und Glaskörper. Diese anormale Adhäsion verursacht ein Makulaödem und dadurch bedingtes Verzerrtsehen und Visusminderung. Bei längerem Bestehen können strukturelle Veränderungen der äußeren Netzhaut und des Pigmentepithels entstehen, was zu einer bleibenden Visusminderung führt.

\subsection{Pars-plana-Vitrektomie}

\subsubsection{Definition}

Die Vitrektomie ist ein mikrochirurgisches Operationsverfahren zur Entfernung des Glaskörpers. Sie ist eine Voraussetzung für chirurgische Manipulationen an der Netzhaut bei verschiedenen Netzhauterkrankungen.

Die Vitrektomie kann vollständig oder teilweise (core vitrectomy) erfolgen. Als Ersatz für den Glaskörper kann eine Tamponade mit inerten Gasen, Luft, Silikonöl oder Ringerlösung eingefüllt werden. Sofern keine bleibende Tamponade mit Öl besteht, wird die eingefüllte Ersatzsubstanz im Glaskörperraum nach und nach durch Kammerwasser ersetzt. Da der Brechungsindex des Glaskörpers mit dem von Wasser nahezu identisch ist, hat eine Vitrektomie keine Auswirkungen auf die Lichtbrechung im Auge.

\subsubsection{Geschichte der Vitrektomie}

Zur Behandlung von Makuladegenerationen, wie Makulaforamen, epiretinaler Gliose und vitreomakulärem Traktionssyndrom, steht heutzutage die Vitrektomie als Therapie der Wahl zur Verfügung. Die Vitrektomie ist nach Katarakt-Operation und Excimerlaser die dritthäufigste ophthalmologische Operationstechnik (Lalezary et al. 2011). 
Robert Machemer gilt als Begründer der Vitrektomie. Seine Assistenzarztzeit absolvierte er von 1962 bis 1966 in der Klinik für Augenheilkunde der Universitätsmedizin Göttingen. Überliefert ist, dass er mit seiner Idee der Vitrektomie keine Unterstützung bei der damaligen Klinikleitung fand. Im Jahr 1966 trat er daher eine Stelle am Bascom Palmer Eye Center (Miami, USA) an. 1968 wurde in Miami erstmals ein prolabierter Glaskörper durch Dr. David Kasner mittels Open-sky-Technik operiert. Bei der Open-sky-Technik wurde unter Verlust der Linse über eine groß angelegte limbale Inzision der Glaskörper entfernt (Augustin und Offermann 2007).

Machemer sah die Bedeutung der Glaskörperentfernung für die Augenheilkunde und begann, an besseren Möglichkeiten der Vitrektomie mit Linsenerhalt zu arbeiten. Durch die Einlagerung von Kollagen kann der Glaskörper nicht einfach abgesaugt werden. Zusammen mit seinem Kollegen Dr. Helmut Büttner entwickelte Machemer ein Verfahren, bei dem der Glaskörper durch gleichzeitiges Aspirieren und Schneiden entfernt werden kann. Mit Hilfe einer Bohrerspitze und einer zylindrischen Röhre konnte er so zunächst an einem Ei kontrolliert das ähnlich dem Glaskörper strukturierte Eiweiß aspirieren (Meyer und Lang 2010). 1971 wurde die von Machemer entwickelte Pars-plana-Vitrekomie (ppV) mit chirurgischem Zugang über die gefäßlose Pars plana eingeführt, bei welcher die Linse erhalten bleibt. Später wurde diese Methode von Klöti weiterentwickelt und präzisiert.

1978 konnte die Vitrektomie als neu etabliertes Verfahren zur Entfernung epiretinaler Gliosen eingesetzt werden. 1991 wurde die ppV auch erstmals als Operationsverfahren bei Makulaforamen eingesetzt (Kelly und Wendel 1991).

\subsubsection{Operationstechniken}

$\mathrm{Zu}$ Beginn der „klassischen“ 20-Gauge-Vitrektomie wird die Bindehaut (Konjunktiva) eröffnet und dann über drei Sklerotomien der Zugang zum Glaskörper geschaffen. Dies erfolgt über die Pars plana des Ziliarkörpers, welche ein eher unfunktionelles und avaskuläres Gewebe am Rand der äußersten Peripherie der Netzhaut ist. Sie liegt ca. 3,5-4 mm hinter dem Limbus. Da beim Zugang über die Pars plana nicht durch die Netzhaut gestochen wird, kommt es nur selten zu einer iatrogenen Netzhautablösung. Über die Zugänge werden drei Instrumente eingeführt:

1) Kanüle für eine Infusion, um den Augapfel tonisiert zu halten

2) Eine Lichtquelle 
3) Kanüle für chirurgisches Werkzeug (Vitrektom, Saugvorrichtungen, Scheren, Greifer, Lasersonden) zum Entfernen des Glaskörpers bzw. zur Manipulation an der Netzhaut.

Der Operateur hält in der einen Hand das Vitrektom, in der anderen Hand die Lichtquelle. Über spezielle Linsen, die zwischen Hornhaut und Operationsmikroskop gebracht werden, wird dem Operateur die Einsicht in den Glaskörper ermöglicht.

Nachdem über viele Jahre die Vitrektomkanüle einen Durchmesser von $20 \mathrm{G}$ hatte, wurden in den letzten Jahren zunehmend dünnere Instrumente mit bis zu $27 \mathrm{G}$ entwickelt. Hierdurch entfällt die Notwendigkeit der Bindehauteröffnung und der Naht der Sklerazugänge. Die Einheit Gauge, welche den Außendurchmesser des Vitrektoms angibt, ist eine amerikanische Einheit für Drähte und gibt die Anzahl der Arbeitsgänge an, bis der Draht seinen Außendurchmesser erreicht. Je häufiger der Draht durch die Drahtziehmaschine gezogen wird, desto dünner wird er, d. h. je größer der Gauge-Wert, desto kleiner der Durchmesser des Vitrektoms.

Tabelle 1: Gauge und korrespondierende Außendurchmesser der Kanüle

\begin{tabular}{|c|c|}
\hline Gauge & Außendurchmesser \\
\hline $19 \mathrm{G}$ & $1,1 \mathrm{~mm}$ \\
\hline $20 \mathrm{G}$ & $0,9 \mathrm{~mm}$ \\
\hline $23 \mathrm{G}$ & $0,6 \mathrm{~mm}$ \\
\hline $25 \mathrm{G}$ & $0,5 \mathrm{~mm}$ \\
\hline $27 \mathrm{G}$ & $0,4 \mathrm{~mm}$ \\
\hline
\end{tabular}

Für die intraokulare Beleuchtung sorgt eine fiberoptische Lichtquelle.

Nach Entfernen des Glaskörpers kann, je nach Krankheitsbild, weiter therapeutisch vorgegangen werden. Makuladegenerationen wie MF, EG, und VMT sind häufige Indikationen zur Vitrektomie. Hierbei kann im Anschluss an die Vitrektomie beispielsweise Gliagewebe von der Netzhaut abgezogen oder die Retina durch Laserkoagulation fixiert werden.

In Studien zeigte sich, dass die Vitrektomie bei Makulaforamen in $58 \%$ zu einem anatomischen Verschluss führt, wobei sich bei $73 \%$ der Patienten eine Visusverbesserung nachweisen ließ (Kelly und Wendel 1991). Aktuellere Studien zeigten Verschlussraten von 84 \% 
(Wakely et al. 2012), nach Phakovitrektomie sogar von $97 \%$ bei 20-G- und $100 \%$ bei 23-GVitrektomie (Krishnan et al. 2013) und eine Visusverbesserung in 94,8 \% (Passemard et al. 2010). Chen konnte eine Verschlussrate von $90 \%$ und eine Visusverbesserung in $73 \%$ nachweisen (Chen 1998).

Eine bekannte Komplikation nach elektiver Vitrektomie ist die Netzhautablösung (Rizzo et al. 2010). Zudem bildet sich oft innerhalb weniger Monate eine Linsentrübung (Katarakt), die eine weitere Operation nötig macht.

\subsubsection{Operationsindikationen}

Bei folgenden Erkrankungen hat sich die Vitrektomie als Therapieverfahren der Wahl etabliert:

- Epiretiale Gliose

- Makulaforamen

- Proliferative Vitreoretinopathie und vitreomakuläres Traktionssyndrom

- Persistierende Glaskörperblutung

- Diagnostische Vitrektomie bei unklarer Entzündung

- Netzhautriss oder Amotio retinae bei Vorhandensein einer Kunstlinse

- Diabetische Makulopathie

- Entfernung von im Glaskörper befindlichen Fremdkörpern oder dislozierter Linse

- Nach penetrierenden Augenverletzungen

- Postoperative oder posttraumatische Veränderungen des Glaskörpers.

\subsubsection{ILM-Peeling}

Das ILM-Peeling beinhaltet die Entfernung der inneren Grenzmembran (internal limiting membrane, ILM) zwischen Glaskörper und Netzhaut. Dies hat sich als Therapiemethode bei Erkrankungen der Netzhautoberfläche wie MF oder EG etabliert. Mit Farbstoffen wie Indocyaningrün (ICG) oder Trypanblau (TB) kann die ILM angefärbt und so sichtbar gemacht.

Eine Studie zeigte, dass bei Makulaforamen-induziertem Zug an der Retina, durch Vitrektomie in Kombination mit ILM-Peeling im Gegensatz zu alleiniger Vitrektomie eine geringere Wiederablösungsrate auftrat (Su et al. 2015). Auch bei MF ohne retinalen Zug zeigte sich das 
ILM-Peeling als eine gute Therapieergänzung (Mester und Kuhn 2000), v. a. bei MF einer Größe von mehr als $300 \mu \mathrm{m}$ (Brooks 2000). Seit 2003 wird bei Operation der EG eine zusätzliche Entfernung der ILM empfohlen, um die Reproliferation der einzelnen Fragmente der EG zu verhindern, da die ILM der EG als Gerüst dient (Park et al. 2003). In einer Studie von Schechet et al. wurden die Patienten unter dem Aspekt des Einflusses der Entfernung der ILM bei EG untersucht. Die Rezidivrate war bei Patienten nach ILM-Peeling niedriger als bei solchen, die sich einer alleinigen Vitrektomie unterzogen (Schechet et al. 2016).

\subsubsection{Peelingfarbstoffe}

Ein Peelingfarbstoff dient der Anfärbung der ILM und der Abgrenzung zur EG, die nicht von den Farbstoffen angefärbt wird. Seit Einführung der Peelingfarbstoffe ist es leichter die ILM atraumatisch abzutragen.

Die in der Studie eingesetzten Peelingfarbstoffe waren:

- Brillant Blue G (Dual Blue; DB)

- Cyanin (Indocyaningrün; ICG)

- Methylthioniniumchlorid (Methylenblau; MB)

- Trypanblau (TB)

- Triphenylmethan (Brilliant Peel; BP)

- und deren Mischungen (ILM-Blau; ILMB, Brilliant Blau; BB)

Wegen möglicher toxischer Schädigungen der Netzhaut wird der Einsatz von Peelingfarbstoffen allerdings auch kritisch gesehen. Als ein Beispiel soll die Wirkung von ICG genauer erklärt werden: ICG ist einer der Farbstoffe, der gezielt nur die ILM und nicht die EG anfärbt. Aber leider hat ICG auch einen Nachteil: In einer Studie von Horio und Horiguchi wurde gezeigt, dass Patienten, die intraoperativ ICG erhalten hatten, ein schlechteres visuelles Outcome hatten als solche ohne ICG. Dies wurde nicht nur durch RPE-Zell-Schaden oder den direkt toxischen Effekt auf die Retina verursacht, sondern auch durch das Anfärben der Ganglienzellen und deren Axone mit ICG (Horio und Horiguchi 2004). Eine Nekrose der Fortsätze der Müller-Zellen auf der retinalen ILM-Seite wurde in einer Studie durch Eckardt et al. jedoch auch ohne Einsatz von ICG beschrieben (Eckardt et al. 1997). In einer weiteren Studie von Sippy et al. zeigte sich bei genauer Untersuchung kultivierter RPE-Zellen, dass ICG die mitochondriale Aktivität der Zellen einschränkte (Sippy et al. 2001). 


\subsubsection{Endotamponade}

Nach der Vitrektomie wird zur Füllung des leeren Glaskörperraums oft eine Endotamponade, bei Makulaforamen meist ein Luft-Gas-Gemisch eingesetzt. Es können hierfür diverse Gase mit unterschiedlicher Verweildauer (C3F8 > C2F6 > SF6 > Luft) gewählt werden. Die genaue Gasfüllung und -konzentration ist wichtig, da so ein postoperativer IOD-Anstieg vermieden werden kann.

Tabelle 2: Verweildauer und Eigenschaften intraokularer Gase (Müller et al. 2010)

\begin{tabular}{lllll}
$\begin{array}{l}\text { Intraokulares } \\
\text { Gas }\end{array}$ & $\begin{array}{l}\text { Maximale Vo- } \\
\text { lumenausdeh- } \\
\text { nung (Faktor) }\end{array}$ & $\begin{array}{l}\text { Obliche verwendete } \\
\text { Konzentration (\%) }\end{array}$ & $\begin{array}{l}\text { Maximaler Ausdeh- } \\
\text { nungszeitpunkt (h) }\end{array}$ & $\begin{array}{l}\text { Verweildau- } \\
\text { er (Tage) }\end{array}$ \\
\hline Luft & 0 & 100 & sofort & $5-7$ \\
\hline SF6 & 2 & 20 & $24-48$ & $10-14$ \\
\hline C2F6 & 3,5 & 16 & $36-60$ & $30-35$ \\
\hline C3F8 & 4 & 12 & $48-72$ & $55-65$ \\
\hline
\end{tabular}

Basierend auf 1,0 ml purem Gas SF6 Schwefelhexafluorid; C2F6 Hexafluorethan; C3F8 Octafluorpropan

Der Sinn der Gasblase ist, den Kontakt zwischen MF und Flüssigkeit möglichst lange zu vermeiden. Das Gas resorbiert sich nach einiger Zeit selbst, anschließend füllt sich der Raum mit Kammerwasser. In Studien erwies es sich als prognoseverbessernd, wenn die Patienten postoperativ eine Kopf-nach-unten-Position einnehmen, vor allem bei MF einer Größe > $400 \mu \mathrm{m}$. Es zeigte sich dadurch eine verbesserte Verschlussrate der Foramina (Lange et al. 2012). Die Angaben zur Dauer variieren von 24 Stunden bis zu wenigen Tagen (Isomae et al. 2002).

Bei Patienten, die kurze Zeit nach Vitrektomie die Kopf-tief-Position nicht einnehmen können, da sie z. B. eine Flugreise antreten müssen, oder bei Patienten mit persistierendem Foramen nach Erstoperation mit Gastamponade, wird statt Gas häufig Silikonöl verwendet. Dies hat den Nachteil, dass es in einer zweiten Operation nach meist 3-6 Monaten wieder entfernt werden muss.

\subsection{Stand der Forschung}

\subsubsection{IOD-Entwicklung unmittelbar postoperativ}

In verschiedenen Studien wurde bereits die IOD-Änderung nach Durchführung einer Vitrektomie untersucht - allerdings ging es hierbei immer um die Möglichkeit einer IOD-Erhöhung. 
Kurzfristig nach durchgeführter ppV sind IOD-Spitzen (englisch: peak) möglich und können durch Verschluss der retinalen Blutversorgung und Ischämie des Sehnervs sowie der Makula $\mathrm{zu}$ einer schwerwiegenden Visusminderung führen. Diese IOD-Spitzen wurden in verschiedenen Studien untersucht, welche im Folgenden dargestellt werden sollen.

Bereits 1976 untersuchten Weinberg et al. den IOD-Anstieg nach erfolgter ppV an 118 Augen in einer retrospektiven Studie mit einer Nachbeobachtungszeit von 12 Monaten. Unter den Pat. waren $43 \%(\mathrm{~N}=51)$ Diabetiker und $57 \%(\mathrm{~N}=67)$ ohne Diabetes in der Anamnese. Von allen Patienten entwickelten 33 (28\%) postoperativ erhöhte IOD-Werte. Unter den 33 Pat. waren 25 diabetische Augen (49\% aller Diabetiker) und 8 nicht-diabetische Augen (12\% aller Patienten ohne Diabetes). Unter den Patienten mit erhöhten IOD-Werten war der IODPeak bei $67 \%(\mathrm{~N}=22)$ in der dritten Woche nach ppV, insgesamt waren IOD-Spitzen von Tag 1 bis Woche 45 festzustellen. In diabetischen Augen konnte ein höheres Risiko einer IOD-Erhöhung gesehen werden als in solchen ohne Diabetes. Die Autoren erklärten sich den IOD-Anstieg postoperativ durch verschiedene Faktoren: u.a. durch eine Neovaskularisation der Iris mit konsekutiver Zunahme von fibrovaskulärem Gewebe im Kammerwinkel; eine Verlegung des Kammerwasserabflusses durch fragmentierte, alte Erythrozyten einer vorangegangenen Blutung; eine mechanische Verlegung des Kammerwasserabflusses durch Blutungen oder Hämolyse (Hämoglobin-beladene Makrophagen) oder durch nach Linsenentfernung zurückgebliebene Linsenfragmente (Weinberg et al. 1976). Auch eine entzündliche Komponente nach Vitrektomie kann über verminderten Kammerwasserabfluss zu einer IOD-Erhöhung führen. Nach Silikonöltamponade ist ebenfalls das Risiko eines IODAnstiegs erhöht. Thompson et al. (1996) untersuchten in einer prospektiven Kohortenstudie an 150 Augen die Inzidenz des IOD-Anstiegs unter TGF- $\beta$-Gabe (rekombinant und bovin) und Placebo bis 3 Monate postoperativ bei MF. Sie zeigten, dass manche Augen nach 2-14 Tagen einen erhöhten IOD aufwiesen. Eine TGF-ß-Applikation intraoperativ, insbesondere das rekombinante, erhöhte das Risiko eines IOD-Anstiegs. Aber nicht nur TGF- $\beta$, sondern auch die Vitrektomie selbst und die Expansion der Gasendotamponade wurden ursächlich für den IOD-Anstieg gesehen. Über TGF- $ß$ wird vermutet, dass es die Kammerwasserproduktion stimuliert, über vermehrte Entzündung den Kammerwasserabfluss senkt und einen direkten Effekt auf das TMW hat (Thompson et al. 1996). Han et al. (1989) konnten in einer prospektiven Studie mit 222 Patientenaugen eine Inzidenz eines relevanten IOD-Anstiegs (auf > $30 \mathrm{mmHg}$ ) von 35,6\% der vitrektomierten Augen ermitteln. Ursächlich wurden die Operationsmechanismen wie operative Skleraeindellung (scleral buckling) und FundusPhotokoagulation sowie Entfernung der Linse (Verstopfung des TMW durch Linsenreste und 
Entzündung postoperativ) und eine postoperativ vermehrte Fibrinbildung nachgewiesen (Han et al. 1989). Chen und Thompson (1997) untersuchten in einer retrospektiven Studie mit 171 Patientenaugen den Einfluss intraokularer Gastamponade nach ppV auf den IOD, von denen $43 \%(\mathrm{~N}=74)$ einen erhöhten IOD aufwiesen, welcher bei $88 \%(\mathrm{~N}=65)$ in den ersten 24 Stunden auftrat und unter medikamentöser Therapie (Kammerwassersuppression) innerhalb 24-72 Stunden zu beherrschen war. Steigendes Patientenalter, dehnbare Gaskonzentrationen, Einsatz von Perfluoropropan (C3F8) und operative Skleraeindellung waren mit einem erhöhten IOD assoziiert (Chen und Thompson 1997). Desai et al. (1997) untersuchten in einer prospektiven Studie mit 25 Patientenaugen mit unterschiedlichen Grunderkrankungen (Glaskörperblutungen, entzündliche Glaskörpertrübungen, EG, Irvin-Gass-Syndrom) den Kurzzeiteffekt der ppV auf den IOD bis maximal 24 Stunden postoperativ. Nach 2 Stunden konnte bei $92 \%(\mathrm{~N}=23)$ ein erhöhter IOD festgestellt werden, bei $80 \%(\mathrm{~N}=20)$ mit einem IODAnstieg um mindestens $5 \mathrm{mmHg}$, bei $39 \%$ lag der IOD bei $30 \mathrm{mmHg}$ und mehr, was $36 \%$ aller untersuchten Augen ausmachte. $40 \%(\mathrm{~N}=10)$ der Studienteilnehmer benötigten eine medikamentöse Therapie zur Drucksenkung. Auch nach 6 Stunden war der IOD signifikant erhöht, jedoch bereits rückläufig, weshalb die Autoren schlussfolgerten, dass der beste Zeitpunkt zur IOD-Messung 2 Stunden nach ppV (in den ersten 24 Stunden postoperativ) ist. Panretinale Photokoagulation und Blutungen intraoperativ führten, anders als zuvor beschrieben, zu keinem signifikanten IOD-Anstieg. Ursächlich vermuteten die Autoren eine mechanische Verlegung des TMW durch fragmentierte Erythrozyten oder Entzündungszellen, jedoch sahen sie auch die ppV selbst als Ursache für eine kurzfristige IOD-Erhöhung, da es z. B. auch bei EG zu einem IOD-Anstieg kam (Desai et al. 1997). Chen (1998) untersuchte an 40 Patientenaugen das Risiko einer Glaukomentwicklung nach alleiniger ppV bei MF bis 3 Monate postoperativ. Eine passagere IOD-Erhöhung konnte bei $52 \%(\mathrm{~N}=21)$ der Teilnehmer festgestellt werden, $22 \%$ der Patienten $(\mathrm{N}=9)$ entwickelten einen IOD-Anstieg auf über $30 \mathrm{mmHg}$ nach 2-4 Stunden und erhielten eine medikamentöse Behandlung, worunter sich der IOD normalisierte. 7,5\% $(\mathrm{N}=3)$ der Patienten brauchten jedoch auf längere Zeit eine drucksenkende Therapie. Mögliche Erklärungen für den IOD-Anstieg waren: fortgeschrittenes Alter der Studienteilnehmer (87\% über 61 Jahre), bei denen die bereits geminderte Filtrationsfunktion im TMW durch operatives Trauma sowie postoperative Entzündung den IOD-Anstieg verursachen; strikte Einhaltung der Kopf-tief-Position nach ppV bei MF mit Einschränkung der Filtrationsfunktion durch Aderhautabhebung und konsekutive Pupillendilatation in dieser Position mit Verschluss des Kammerwinkels. Dies wurde vermutet, da 7 von 12 Patienten, die postoperativ gonioskopisch untersucht wurden, 
einen engen Kammerwinkel aufwiesen. Des Weiteren erklärte sich Chen den IOD-Anstieg durch die Gasausbreitung der Endotamponade in den ersten 24 Stunden. Ein signifikanter Einfluss des Alters, des Geschlechts, des Linsenstatus, des MF-Verschlusses und der Rasse konnte jedoch bei vorliegend kleiner Patientenklientel nicht auf den IOD-Anstieg nachgewiesen werden (Chen 1998). Anderson et al. (2006) untersuchten die Inzidenz von IODSpitzen nach ppV und/oder Skleraeindellung an 202 Augen (rhegmatogene Netzhautablösung, diabetische Retinopathie, MF, altersbedingte Makuladegeneration) bis 1 Tag postoperativ. 8,4\% der Patienten $(\mathrm{N}=17)$ wiesen nach 5-12 Stunden, 14,8 \% $(\mathrm{N}=30)$ nach einem Tag postoperativ einen erhöhten IOD von über $29 \mathrm{mmHg}$ auf. Es konnte kein erhöhtes Risiko eines IOD-Anstiegs in Bezug auf Diagnose, Endotamponade, Silikonöl, Skleraeindellung und Endolaser eruiert werden. Schlussfolgerung der Autoren war, dass die Inzindenz einer IODSpitze nach $\mathrm{ppV}$ niedrig ist und mit Medikamenten, die eine Kammerwassersuppression induzieren, beherrscht werden kann (Anderson et al. 2006). Benz et al. (2004) konnten in einer prospektiven Studie an 50 Patientenaugen die Wirkung von kammerwassersupprimierenden Medikamenten postoperativ im Vergleich zu Placebo untersuchen. Ein IOD $>25 \mathrm{mmHg}$ konnte bei $75 \%$ der Patienten ohne Therapie im Vergleich zu $9 \%$ mit Therapie nach 5 Stunden festgestellt werden, weshalb gerade bei Risikopatienten (Glaukom, intraokuläre Hypertension) der IOD auch 5 Stunden nach ppV gemessen werden sollte (Benz et al. 2004). Hasegawa et al. (2012) untersuchten in einer prospektiven Studie die IOD-Erhöhung nach 5 und 24 Stunden post-ppV mit kombinierter Kataraktextraktion bei rhegmatogener Netzhautablösung $(\mathrm{N}=34)$ im Vergleich zu MF ( $=12)$. Die Inzidenz des IOD-Anstiegs lag nach 5 Stunden bei Netzhautablösungen bei 14,7 \% und $0 \%$ bei MF, nach 24 Stunden 38,2 \% bei Netzhautablösungen und 8,3\% bei MF. Dass die Inzidenz der IOD-Erhöhung nach 24 Stunden höher war als nach 5 Stunden, erklärten sich die Autoren durch die eingesetzte Gasendotamponade SF6, die nach 24-48 Stunden postoperativ ihre maximale Ausdehnung erreicht. Alter der Patienten, intraoperative Photolaserkoagulation und kombinierte Kataraktchirurgie standen in keiner signifikanten Verbindung zu postoperativen IOD-Spitzen. Ursächlich für den erhöhten IOD wurde die Theorie der mechanischen Verlegung des TMW durch retinales Pigmentepithel und/oder Entzündungszellen gesehen. Auch eine exzessive Kammerwasserbildung wurde in Erwägung gezogen (Hasegawa et al. 2012). Framme et al. (2012) untersuchten in einer prospektiven Studie mit 851 Patienten mit breitem Krankheitsspektrum und unterschiedlichen Endotamponaden den IOD bis 48 Stunden postoperativ. Bei Silikonölendotamponade zeigten sich bereits nach 3 Stunden IOD-Spitzen (21,8 mmHg), die Gasendotamponaden wiesen hingegen erst ab 24 Stunden und danach einen IOD-Anstieg auf. 
Eingesetzte Laser- und Kryotherapie können ebenfalls einen Einfluss auf die IOD-Erhöhung genommen haben. Die Autoren empfahlen daher eine engmaschige Kontrolle nach 4-6 Stunden, v. a. nach Silikonöltamponade und innerhalb der ersten 24 Stunden nach Gasinfiltration (Framme et al. 2012).

\subsubsection{IOD-Entwicklung im Langzeitverlauf}

Da die Indikationsstellung zur ppV sehr vielseitig ist und sie mittlerweile die dritthäufigste ophthalmologische Operationstechnik darstellt, ist es wichtig, die Langzeitkomplikationen dieses Eingriffs genauer zu kennen. Einige Studien vermuten eine Langzeiterhöhung des IODs nach Vitrektomie, was im Verlauf das Risiko für die Entwicklung eines sekundären Glaukoms steigern würde. Andere Studien konnten diesen Zusammenhang jedoch nicht nachweisen. Die Ergebnisse der Forschungsarbeiten sind kontrovers und sollen im Folgenden dargestellt werden.

Chang (2006) hatte als Erster den Verdacht, dass sich sekundäre Offenwinkelglaukome als Langzeitfolge der Vitrektomie entwickeln können. Seine Studie schloss 453 Patienten mit breitem Krankheitsspektrum ein, welche durchschnittlich über einen Zeitraum von 56,9 Monaten untersucht wurden. Ursächlich vermutete er oxidativen Stress auf das TMW, wodurch konsekutiv der IOD und das Risiko für Offenwinkelglaukome steigen sollten (Chang 2006). Im Auge besteht physiologisch ein Sauerstoffgradient, welcher an der Netzhautoberfläche am höchsten ist und bis zur Rückseite der Linse abnimmt. Der physiologische Sauerstoffgradient wird normalerweise durch die gelartige Masse des Glaskörpers und die Linse über antioxidative Mechanismen (Glutathion, Katalase, oxidative Phosphorylierung) abgebaut. Nach Vitrektomie steigt der Sauerstoffgradient an der Linse auf sein Dreifaches, bleibt bis 10 Monate nach ppV persistierend, wirkt bei phaken Patienten vermehrt auf die Linse und kann zu Kernkatarakt führen (Holekamp et al. 2005), da die Proteinoxidation im Kern der Linse beginnt und die eigentlich relativ hypoxische Linse nicht ausreichend antioxidative Abwehrmechanismen hat um die erhöhten Sauerstoffkonzentrationen zu kompensieren. Bei vitrektomierten Pseudophaken kann auch die Linse den Sauerstoff nicht mehr metabolisieren. Dieser gelangt daher durch die vordere Augenkammer zum Kammerwinkel und könnte dort zu einer IOD-Erhöhung führen. Deshalb wird vermutet, dass das Risiko für Offenwinkelglaukome erhöht ist (Siegfried et al. 2010). Chang schätzte die Inzidenz des Offenwinkelglaukoms nach Vitrektomie auf 15-20\% bei pseudophaken Patienten (Chang 2006). Koreen et al. (2012) untersuchten in einer retrospektiven Kohortenstudie 285 
vitrektomierte Augen und beschrieben eine Inzidenz des Offenwinkelglaukoms von 11,6\% $(\mathrm{N}=33$ ) nach mindestens 6 Monaten Follow-up (FU). Der einzige Einflussfaktor, der einen signifikanten Einfluss auf den IOD zeigte, war auch hier die Kataraktextraktion und die dadurch bedingte Pseudophakie. Changs Hypothese des oxidativen Stresses am TMW und an den Kammerwasserausflusskanälen wurde hier ebenfalls angenommen (Koreen et al. 2012). Luk et al. (2009) untersuchten in einer retrospektiven Fallserie 101 Patientenaugen, die aufgrund von EG und MF vitrektomiert wurden, mit einem FU von mindestens 6 Monaten. Hierunter entwickelten $7,9 \%(\mathrm{~N}=8)$ der Patienten ein Offenwinkelglaukom $(13 \%$ in der Gruppe der pseudophaken, $2 \%$ in der Gruppe der phaken vitrektomierten Augen). Auch hier wurde die Hypothese des oxidativen Stresses auf das TMW mit protektivem Effekt der Linse angenommen (Luk et al. 2009). Eine weitere retrospektive Muticenterstudie (2014) untersuchte 198 Augen, die aufgrund einer EG vitrektomiert wurden mit einem FU von mindestens 12 Monaten $(47,3 \pm 24$ Monate). Hier wurden nach unkomplizierter ppV bei Patienten mit Pseudophakie und positiver Familienanamnese für Offenwinkelglaukome ebenfalls erhöhte IOD-Werte gemessen. In der Gruppe der vitrektomierten Augen wiesen 19,2\% $(\mathrm{N}=38)$ einen erhöhten IOD nach ppV auf, in der Kontrollgruppe 4,5\% (N=9). Bei 18 Patienten konnte eine positive Familienanamnese für Glaukome nachgewiesen werden, von denen $55,6 \%(\mathrm{~N}=10)$ im Verlauf einen deutlich erhöhten IOD entwickelten. Auch hier formulierten die Autoren die Hypothese des gesteigerten oxidativen Stresses nach Vitrektomie. Bezüglich des erhöhten Risikos bei positiver Familienanamnese wurde eine genetische Prädisposition und dadurch gesteigerte Empfindlichkeit für oxidativen Stress auf das TMW vermutet (Wu et al. 2014). Auch Toyokawa et al. (2015) konnten bei 767 Patientenaugen in einer retrospektiven Fallstudie nach Vitrektomie mit kombinierter Kataraktextraktion und breitem Krankheitsspektrum (rhegmatogene Netzhautablösung, EG, MF, Glaskörperblutung, subretinale Blutung, vitreomakuläres Traktionssyndrom, Glaskörpertrübung und Retinoschisis) auf lange Zeit (47,8 \pm 25,3 Monate) die Entwicklung eines späten IOD-Peaks und okulärer Hypertension bei 4,2\% der Pat. ( $\mathrm{N}=32$; 31 von 32 unilaterale IOD-Spitze) feststellen. Ein signifikanter Zusammenhang zwischen IOD-Anstieg und Krankheitsbild, Geschlecht und Vitrektomgröße konnte nicht nachgewiesen werden. Auch diese Autoren nutzten die Hypothese des gesteigerten oxidativen Stresses auf das TMW. Des Weiteren vermuteten sie einen Einfluss der Gastamponade auf den IOD. Augen, die eine Gastamponade erhielten, zeigten in 5,2\% $(\mathrm{N}=25 / 478)$, die ohne Gastamponade in 2,4\% $(\mathrm{N}=7 / 289)$ einen erhöhten IOD, jedoch konnte mathematisch kein signifikanter Unterschied zwischen Augen mit und ohne Gastamponade in Bezug auf die Entwicklung einer okulären Hyertension 
festgestellt werden (Toyokawa et al. 2015). Fujikawa et al. (2014) untersuchten in einer retrospektiven Studie die Langzeit-IOD-Unterschiede (29,3 Monate) nach Vitrektomie für MF $(\mathrm{N}=61)$ und EG $(\mathrm{N}=57)$ im Vergleich zu den nicht-vitrektomierten Kontrollaugen. Sie fanden einen signifikanten IOD-Unterschied zwischen operiertem und Kontrollauge bei der Abschlussuntersuchung in der Gruppe der MF (0,7 mmHg), jedoch nicht bei EG $(0,3 \mathrm{mmHg})$. MF hatten ein signifikantes Risiko eines IOD-Anstiegs nach ppV, die meisten vitrektomierten MF-Augen unterzogen sich jedoch auch einer kombinierten Kataraktextraktion, was die Autoren wieder durch oxidativen Stress am TMW erklärten. Auch die intraoperative Gastamponade wurde als Risikofaktor für den IOD-Anstieg diskutiert, da Gastamponaden erhöhte Konzentrationen inflammatorischer Zytokine oder das Verstopfen des TMW mit Entzündungszellen verursachen können, was durch die Kopf-tief-Position verschlimmert werden kann (Fujikawa et al. 2014). In einer weiteren retrospektiven Fallserie (2007) wurden 25 Pat. mit Makulaödem, bedingt durch diabetische Retinopathie oder Verschluss der Retinavene, mit Triamcoloninjektion vitrektomiert. Die Autoren wiesen nach, dass vitrektomierte Augen Triamcolon-dosisabhängige IOD-Anstiege aufwiesen. Diese IOD-Spitzen waren in den ersten postoperativen Tagen am höchsten, normale Werte konnten erst nach einigen Monaten (3-6 Monate) nachgewiesen werden (Yamashita et al. 2007). Lalezary et al. (2014) konnten in einer prospektiven Studie mit 40 Patienten den Einfluss der ppV bei EG, MF und Glaskörpertrübung unter anderem auf den IOD innerhalb eines Jahres untersuchen. Diese konnten einen signifikanten Anstieg des IODs von pseudophaken Augen nach Vitrektomie nachweisen (Lalezary et al. 2014). Mi und Thompson (2015) untersuchten ebenfalls in einer allerdings retrospektiven Fallserie mit 234 Patienten (ohne Anamnese eines Glaukoms oder Diabetes) den Langzeit-IOD (52,2 $\pm 36,7$ Monate) in Bezug auf die Entwicklung eines Offenwinkelglaukoms nach Vitrektomie bei EG und MF. Bei 2,6\% der Patienten $(\mathrm{N}=6)$ konnte die Erstdiagnose eines Offenwinkelglaukoms gestellt werden, unter denen 1,7\% $(\mathrm{N}=4)$ auch am Partnerauge ein neues Offenwinkelglaukom entwickelten. Es konnte jedoch kein signifikanter IOD-Unterschied zwischen phaken und nicht-phaken vitrektomierten wie auch den Kontroll-Augen festgestellt werden. Diese Inzidenzangaben sind vergleichbar mit denen in der sonstigen Literatur. Interessanterweise war der gemessene IOD der vitrektomierten Augen in der Anfangsmessung, nach 3 und 6 Monaten signifikant niedriger als der des Partnerauges. Ein und zwei Jahre nach erfolgter Vitrektomie war der gemittelte IOD weiter niedriger, jedoch nicht mehr statistisch signifikant. In der Endmessung konnten nach Einsatz von ICG als Peelingfarbstoff signifikant ( $<$ 0,05) höhere IOD-Werte als in Augen ohne ICG-Einsatz gemessen werden (Mi und Thompson 2015). Auch Yu et al. (2010) 
konnten in einer 5-Jahres-Nachbeobachtung einer retrospektiven Fallserie mit 441 Patienten keine erhöhte Inzidenz für die Entstehung eines Offenwinkelglaukoms nachweisen. Jeweils $4,31 \%$ der vitrektomierten Augen (jeweils $\mathrm{N}=19$ ) entwickelten auf lange Zeit ein Offenwinkelglaukom oder eine okuläre Hypertension, unter den Kontrollaugen wiesen 2,49 \% $(\mathrm{N}=11)$ ein Offenwinkelglaukom und 2,95\% ( $\mathrm{N}=13)$ eine okuläre Hypertension auf. Dies erbrachte keinen statistisch signifikanten Unterschied. Auch der Linsenstatus stellte keinen Einflussfaktor für die Entstehung eines Offenwinkelglaukoms dar. Die Autoren schlussfolgerten daraus, dass die Vitrektomie ein sicheres Operationsverfahren ohne gesteigertes Risiko für die Entwicklung eines Offenwinkelglaukoms darstellt (Yu et al. 2010). Yamamoto et al. (2016) untersuchten in einer retrospektiven Studie mit stringenten Ein-und Ausschlusskriterien die IOD-Veränderung bis ca. 2 Jahre post-ppV für Retinaablösung $(\mathrm{N}=54), \mathrm{MF}$ $(\mathrm{N}=75)$ und EG $(\mathrm{N}=117)$. Die Studie ergab auf lange Zeit einen deutlichen Druckanstieg für Retinaablösungen, für MF und EG blieb der IOD jedoch stabil. Bei den Retinaablösungen entfernten die Operateure den gesamten Glaskörper, bei EG und MF wurde nur eine Kernvitrektomie (core vitrectomy) durchgeführt, weshalb die Autoren die Hypothese des oxidativen Stresses auf das TMW wieder unterstützen konnten, da bei einer Kernvitrektomie der Glaskörper nur teilweise entfernt wird. Ein Einfluss der Gastamponade auf den IOD konnte nicht festgestellt werden (Yamamoto et al. 2016). Ki et al. (2013) untersuchten in einer retrospektiven Fallstudie 85 Patientenaugen in einem Nachbeobachtungszeitraum von 21,9 \pm 11,1 Monaten, die sich bei MF und EG einer ppV mit kombinierter Phakoemulsifikation und Linsenimplantation unterzogen. Hier konnte nach 3 Monaten ein signifikant niedrigerer IOD in den operierten Augen im Vergleich zu den Kontrollaugen gemessen werden (Differenz $1,17 \mathrm{mmHg}$ ), welcher jedoch im Verlauf wieder langsam auf das präoperative Level anstieg. Nur 2 operierte Augen wiesen einen deutlichen IOD-Anstieg 6 Monate postoperativ auf, welcher sich jedoch im Verlauf ohne Therapie normalisierte. Ein signifikanter IOD-Unterschied zwischen operiertem und Kontrollauge sowie Augen mit und ohne Endotamponade konnte nicht entdeckt werden (Ki-I et al. 2013). Lalezary et al. (2011) untersuchten in einer retrospektiven Kohortenstudie mit 101 Patientenaugen über durchschnittlich 49 Monate (12105 Monate) den IOD nach Vitrektomie bei Glaskörperblutung (37\%), EG (36\%) und MF (14\%), sowie chronischem Makulaödem und Traktionsnetzhautablösung. $62 \%$ der Patienten $(\mathrm{N}=63)$ hatten Diabetes als Grunderkrankung. Die Autoren konnten keinen signifikanten IOD-Anstieg protokollieren. Auch Pseudophakie und Diabetes als Grunderkrankung hatten keinen Einfluss auf den IOD. 35 Augen zeigten im Langzeitverlauf erniedrigte IOD-Werte, 52 einen IOD-Anstieg und 14 Augen blieben auf gleichem Niveau stabil. Die Inzidenz eines 
erhöhten IOD lag nach 4 Jahren bei $7 \%$, nach 8 Jahren bei $34 \%$, jedoch konnte kein signifikanter Unterschied zum Kontrollauge festgestellt werden. Über die Augen mit erniedrigten IOD-Werten waren die Autoren selbst erstaunt, da sie, entsprechend der bekannten Tendenz eines mit dem Alter leicht steigenden Drucks, eher mit einem kleinen Anstieg des IOD gerechnet hatten. Dies erklärten sich die Autoren durch eine verminderte Kammerwasserproduktion und vermehrtem Kammerwasserabfluss (Lalezary et al. 2011). Erhöhter oxidativer Stress kann zu arterieller Konstriktion führen, was wiederum den Blutfluss zum Ziliarkörper und damit die Kammerwasserproduktion vermindert. Des Weiteren könnte bedingt durch die Vitrektomie die sklerale Festigkeit verändert und damit auch der episklerale Venendruck und konsekutiv der IOD beeinflusst werden. Jedoch wurde noch in keiner Studie untersucht, ob der Druck auf lange Zeit tatsächlich sinkt.

Zusammenfassend gibt es kontroverse Ergebnisse, wie sich der IOD mittel- bis längerfristig nach Vitrektomie verhält und welche Einflussfaktoren hierbei eine Rolle spielen. Auch über den mittelfristigen Einfluss der Vitrektomgröße auf den IOD gibt es unterschiedliche Studienergebnisse. Neben den zahlreichen Studien, die sich mit einer möglichen Druckerhöhung bzw. Entstehung eines Glaukoms beschäftigen, fehlen Studien, die gezielt einen längerfristigen drucksenkenden Effekt untersuchen. Dies ist Anlass für unsere retrospektive Untersuchung.

\subsubsection{Einfluss der Vitrektomgröße (20 G, 23 G) auf den IOD}

Nach Einführung der ppV durch Machemer 1971 und nachfolgende Etablierung der 20-GVitrektomie mit Bindehauteröffnung, konnten Fujii et al. 2002 die nahtlose 25-G-Operation einführen (Fujii et al. 2002b; Fujii et al. 2002a). Eckardt führte 2005 die 23-G-Technik ebenfalls ohne Wundnaht ein. Vorteil des etwas dickeren Vitrektoms ist die größere Steifigkeit der Instrumente (Eckardt 2005). Da die Sklerazugänge jetzt nicht mehr genäht werden, kann es durch postoperative Leckage zu einer Hypotonie des Auges nach $23 \mathrm{G}$ und $25 \mathrm{G}$ kommen. Sowohl Eckardt (2005) als auch Singh et al. (2010) konnten einen Tag postoperativ nach 23 G keine Anzeichen einer Hypotonie erkennen (Eckardt 2005; Singh et al. 2010). Fine et al. (2007) untersuchten an 77 Augen bis 3 Monate nach ppV unter anderem den IOD. Hier zeigten sich außer einer postoperativen Hypotonie bei 2,8 \% ( $\mathrm{N}=2)$ der Pat. an Tag eins auch nach drei Monaten bei den meisten Patienten stabile IOD-Werte (Fine et al. 2007). Lott et al. (2008) untersuchten die Kurzzeitkomplikationen bis 4 Wochen nach $23 \mathrm{G}$ ppV an 100 Augen mit breitem Krankheitsspektrum. Unter anderem konnte bei $10 \%$ der Patienten $(\mathrm{N}=10)$ am ersten Tag postoperativ eine intraokuläre Hypotonie festgestellt werden, nach einer Woche 
konnte diese nur noch bei $3 \%(\mathrm{~N}=3)$, nach einem Monat bei $1 \%(\mathrm{~N}=1)$ gemessen werden (Lott et al. 2008). Gupta et al. (2008) untersuchten ebenfalls in einer retrospektiven Fallserie die Kurzzeitkomplikationen nach ppV mit 23-G-Vitrektom an 92 Patientenaugen und konnten eine Hypotonie-Inzidenz von 6,5\% $(\mathrm{N}=6)$ kurzzeitig postoperativ feststellen (Gupta et al. 2008). In einer Studie mit 20-G-ppV operierten Augen konnten Framme et al. (2012 (siehe Teil 1.5.1)) keine lang anhaltende Hypotonie (> 6 Stunden) feststellen (Framme et al. 2012).

Ahn et al. (2012) verglichen in einer retrospektiven Fallserie an 338 Augen die postoperativen IOD-Unterschiede zwischen 20-G- und 23-G-ppV bis einen Monat nach erfolgter Operation. An Tag eins zeigte sich ein erhöhtes Hypertonierisiko in der 20-G- $(21,6 \% 20$ G; 1,8 \% 23 G), ein erhöhtes Hypotonierisiko in der 23-G-Gruppe (23 G $3 \%, 20$ G $0 \%$ ). Nach einer Woche und einem Monat konnten keine signifikanten Unterschiede mehr festgestellt werden. Die IOD-Werte zeigten in der Gruppe der 23-G-operierten Augen einen stabileren Verlauf. Die Autoren konnten daher schlussfolgern, dass die 23-G-ppV stabilere und niedrigere IODWerte in der frühen postoperativen Periode mit sich bringt als die 20-G-Operation. Eine mögliche Erklärung der Hypotonie und fehlenden Hypertonie nach 23-G-ppV ist die nahtlose ppV-Wunde. Die offene Wundfläche dient als Ventil des Druckgradienten und beugt so über kleinste Leckagen einer Hypertonie vor. Als weitere Ursache sahen die Autoren eine ausgeprägtere Entzündungsreaktion nach 20-G-ppV welche, wie bereits in Teil 1.5.1 dargestellt (Chang 2006), über vermehrte Entzündungszellen das TMW und den Ziliarkörper in seiner Funktion beeinflussen. Hierdurch kann es über Behinderung des Kammerwasserabflusses zu einer IOD-Spitze kommen (Ahn et al. 2012). Eine Fallserie von Misra et al. (2009) untersuchte an 100 Augen unter anderem die Auswirkung auf den IOD einen Tag postoperativ im Vergleich $20 \mathrm{G}(\mathrm{N}=50)$ und $23 \mathrm{G}(\mathrm{N}=50)$ an Patienten mit breitem Krankheitsspektrum. Auch hier zeigte sich eine Hypotonie-Inzidenz nach 23-G-ppV bei 4 Patienten, nach 20-GVitrektomie bei keinem. Eine entscheidende Beobachtung war, dass keiner in der 23-G- und 4 in der 20-G-Gruppe eine Hypertonie aufwiesen, was die Autoren schlussfolgern ließ, dass die 23-G-Vitrektomie das Risiko von IOD-Spitzen am ersten Tag postoperativ über Gasundichtigkeiten und verminderte Entzündungsreaktion reduziert (Misra et al. 2009). Krishnan et al. (2013) konnten in einer Fallserie mit 72 phakovitrektomierten Patientenaugen im Vergleich $20 \mathrm{G}$ und $23 \mathrm{G}$ einen Tag postoperativ signifikant höhere IOD-Werte in der Gruppe der $20 \mathrm{G}$ messen. 7 Tage postoperativ waren die IOD-Werte weiter erhöht, jedoch nicht mehr signifikant im Vergleich zur 23-G-Gruppe. Hypotonien konnten in beiden Gruppen nicht nachgewiesen werden. Zwei bis drei Monate postoperativ konnte kein Unterschied zwischen $20 \mathrm{G}$ und $23 \mathrm{G}$ festgestellt werden (Krishnan et al. 2013). In einer prospektiven Studie mit 60 
Patientenaugen von Wimpissinger et al. (2008) wurde als sekundärer Endpunkt auch der IOD zwischen $20 \mathrm{G}$ und $23 \mathrm{G}$ verglichen. Hier konnte in den ersten 2 Tagen postoperativ kein signifikanter Unterschied zwischen den Behandlungsgruppen festgestellt werden. In der 20G-Gruppe wiesen 4 Patienten eine Hypotonie und 8 Patienten eine Hypertonie auf, in der der 23-G-vitrektomierten waren 4 Patienten mit Hypertonie und 8 Patienten mit Hypotonie, womit sich statistisch kein signifikanter Unterschied errechnen ließ (Wimpissinger et al. 2008).

In einer prospektiven Studie von Zhang et al. (2015) untersuchten die Autoren an 149 Augen (20 G: $\mathrm{N}=49 ; 23 \mathrm{G}: \mathrm{N}=97$ ) mit breitem Krankheitsspektrum unter anderem den IOD im Vergleich $20 \mathrm{G}$ und $23 \mathrm{G}$ mit unterschiedlichen Endotamponaden. Der gemittelte IOD präund postoperativ war mit Ausnahme des ersten Tages bis 6 Monate FU zwischen den beiden Gruppen gleich. Es konnte kein statistisch signifikanter Unterschied festgestellt werden. An Tag eins zeigten sich jedoch die IOD-Werte der 23-G-Gruppe statistisch signifikant erniedrigt. Hypotonien konnten $\mathrm{zu} 9 \%$ in der 23-G- und $\mathrm{zu} 2 \%$ in der 20-G-Gruppe festgestellt werden. Ursächlich hierfür vermuteten die Autoren ebenfalls die fehlende Wundnaht und den dadurch bestehenden Druckausgleich über die Wunde. Des Weiteren wurde ein Zusammenhang mit der verwendeten Endotamponade gesehen: Flüssigkeitsgefüllte Augen hatten eine deutlich erhöhte Inzidenz einer postoperativen Hypotonie als solche mit Silikonöl oder Gas (Zhang et al. 2015). 


\subsection{Fragestellung}

Die vorliegende Studienlage über mittel- bis langfristige IOD-Änderungen nach ppV bei Makulaerkrankungen ist sehr kontrovers (siehe Kap. 1.5). Praktisch alle bisher veröffentlichten Arbeiten nehmen als Ausgangshypothese eine Steigerung des Augeninnendrucks an. Jedoch gibt es bisher keine Untersuchungen, ob ein längerfristiger drucksenkender Effekt eintritt. Beschrieben wurde dieses Phänomen bisher in zwei Studien (Lalezary et al. 2011; Mi und Thompson 2015), welche eigentlich eine potentielle Drucksteigerung untersuchten.

Ziel der vorliegenden retrospektiven Studie ist es, die mittel- bis längerfristige IOD-Änderung nach erfolgter ppV unter der Hypothese einer ppV-induzierten Drucksenkung zu untersuchen.

$\mathrm{Zu}$ beantwortende Fragen sind:

1) Sinkt der IOD mittel- bis längerfristig im operierten Auge im Vergleich zum nichtoperierten Kontrollauge zu verschiedenen Zeitpunkten postoperativ? Hierfür wird als primärer Endpunkt die IOD-Änderung nach 6-12 Monaten festgelegt. Für die sekundären Endpunkte wird ein Zeitraum von 3-6 und 12-24 Monaten festgelegt.

2) Welche Einflussfaktoren (Linsenstatus, Vitrektomgröße, Endotamponade, Geschlecht, Alter, sphärisches Äquivalent, Anzahl der drucksenkenden Augentropfen, Operation) können eine Rolle bei der vermuteten IOD-Senkung spielen? 


\section{Material und Methoden}

Die Daten für diese retrospektive Patientenstudie (Fallserie) wurden durch Auswertung der Krankenakten im Archiv der Universitätsmedizin Göttingen erhoben. Vor Studienbeginn wurde der Studienplan der Ethikkommission zur Begutachtung vorgelegt. Gegen die Doktorarbeit bestanden keine Bedenken (Anhang § 6.1).

\subsection{Patientenkollektiv}

In diese Studie eingeschlossen wurden Patienten, die sich in der Klinik für Augenheilkunde der Universitätsmedizin Göttingen einer Pars-plana-Vitrektomie unterzogen haben. Als Operationsindikationen waren die Makulaerkrankungen epiretinale Gliose, vitreomakuläres Traktionssyndrom und Makulaforamen zugelassen. Patienten mit einer kombinierten Operation (Vitrektomie und Katarakt-Operation) wurden ausgeschlossen.

Folgende Studienparameter wurden untersucht:

- Linsenstatus präoperativ (d. h. phak oder pseudophak)

- Intraoperative Endotamponade (mit Gas, Luft oder Ringer-Lösung)

- Verwendete Peelingfarbstoffe

- Applikation von steroidhaltigen oder IOD-senkenden Augentropfen

- Größe des Vitrektoms (20 G, 23 G)

- Sphärisches Äquivalent

Der IOD des nicht-operierten Auges wurde als Kontrollparameter ebenfalls erfasst. Präoperative IOD-Werte wurden zwischen den Werten der Erstvorstellung (meist etwa einen Monat vor Operation) und den Werten am Tag vor der Operation gemittelt. Als Linsenstatus wurde der präoperative Linsenstatus, d. h. phak oder pseudophak, welcher in der Akte notiert war, benutzt. Verwendete Endotamponaden nach ppV waren inerte Gase wie C2F6 und SF6 und Luft. Peelingfarbstoffe zur Anfärbung der ILM und besseren Abgrenzung der epiretinalen Gliosen waren Dual Blue, Methylenblau, Trypanblau, ILM-Blau, Brilliant Blau, Brilliant Peel und Indocyaningrün. Das sphärische Äquivalent wurde jeweils aus gemessener Sphäre und Zylinder der Brille vor geplanter Operation berechnet:

$$
\text { Sphärisches Äquivalent }=\text { Sphäre }+1 / 2 \text { Zylinder }
$$

Änderungen des sphärischen Äquivalents nach Operation wurden nicht in der Studie berücksichtigt. 
Von der Studie ausgeschlossen wurden Patienten mit:

- Zu kurzer Nachbeobachtungszeit (<2 Monate)

- Einem Alter unter 18 Jahren

- Bekannter Steroide-Response (IOD-Anstieg nach Steroidgabe)

- Z.n. Uveitis

- Z.n. schwerem Trauma

- Bekanntem Glaukom

- Intra- oder postoperativer Katarakt-Operation

- Vorangegangener Vitrektomie

- Ausgiebiger Netzhaut-Kryokoagulation (> 2 Uhrzeiten)

- Kombinierter Cerclage oder Plombe

- Intraoperativer panretinaler Laserkoagulation

- Öltamponade (Silikonöl), Steroidinjektion (auch Triamcolon) oder Thrombozytenkonzentrat intraoperativ

- Ablatio Retinae

- Glaskörperblutung/Makulablutung

- Diabetischer Retinopathie und Makulaödem

\subsection{Datenerhebung}

Die Datenerhebung fand ausschließlich im Archiv der Universitätsklinik Göttingen statt. Hierfür wurden 596 Akten von Patienten der UMG gesichtet, die aufgrund einer epiretinalen Gliose, eines vitreomakulären Traktionssyndroms oder eines Makulaforamens operiert wurden. In die Studie eingeschlossen wurden 249 Patienten.

Neben den o. g. Studienparametern wurden folgende Patientendaten erhoben:

- Patientennummer, Alter, Geschlecht

- Diagnose (Makulaforamen, Epiretinale Gliose, Vitreomakuläres Traktionssyndrom)

- Operationsdatum

- Operationsseite (rechts/links)

- Untersuchungsdatum und -zeitpunkt

Um eine statistische Auswertung des zeitlichen Verlaufs des IOD zu ermöglichen, wurden die Untersuchungszeitpunkte in Gruppen zusammengefasst. Wurde mehr als eine Visite in 
einem der unten genannten Zeitintervalle dokumentiert, wurde der Mittelwert aller vorliegenden dokumentierten IOD verwendet.

Die Zeitintervalle waren folgende:

- $\quad$ bis zu 6 Tage vor erfolgter OP (prä-OP)

- $\quad$ bis zu 6 Tage nach erfolgter OP (post-OP)

- $\quad$ nach Entlassung bis 3 Monate nach OP $($ Entl $<$ Monat $\leq \mathbf{3})$

- $\quad$ nach 3 Monaten post-OP bis $\leq 6$ Monate nach OP $(3<$ Monat $\leq 6)$

- $\quad$ nach 6 Monaten post-OP bis $\leq 12$ Monate nach OP $(\mathbf{6}<$ Monat $\leq \mathbf{1 2})$

- $\quad$ nach 12 Monaten post-OP bis $\leq 24$ Monate nach OP $(\mathbf{1 2}<$ Monat $\leq \mathbf{2 4})$

- $\quad$ nach 24 Monaten post-OP $(24<$ Monat $)$

In den Akten waren die prä-OP Werte bis zu 6 Tage vor der Operation angegeben. Die PostOP-Werte wurden bis zu 6 Tage nach Operation gemessen. Die am Entlassungstag angegebenen Messwerte waren bis 7 Tage nach Operation erhoben worden. Anschließend wurden IOD-Werte zu diversen Zeitpunkten nach der Operation ohne festes Schema bis zu 2 Jahren nach erfolgter Operation dokumentiert.

\subsection{Verwendete statistische Methoden}

Der erhobene Datensatz enthält den dokumentierten Augeninnendruck beider Augen von 249 Patienten vor und nach einer Augenoperation an genau einem Auge. Dies bedeutet, dass bei jedem Patienten ein Datensatz für jeweils das operierte und nicht-operierte Auge erhoben wurde.

Für die statistische Auswertung wurden der Stichprobenumfang (N), der Mittelwert (Mean), der Standardfehler (SEM - standard error of the mean), die Standardabweichung (SD), Maximal - und Minimalwert (MIN, MAX), sowie das obere und untere Konfidenzintervall (UCL - upper confidence limit, LCL - lower confidence limit) berechnet.

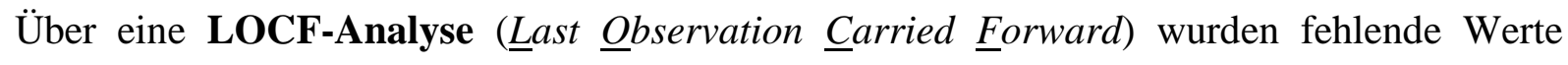
anhand von Berechnung linear interpoliert, wenn Werte vorher und nachher vorhanden waren oder fortgeschrieben, wenn nachfolgend keine weiteren Werte mehr dokumentiert waren. Verwendet wurden hierfür neben der prä-OP-Visite nur die vorher definierten Zeitintervalle. 
Durch die Interpolation wurden fehlende Messwerte für jeden Zeitpunkt aufgefüllt, so dass für die statistische Auswertung immer die vollständige Anzahl an untersuchten Patientenaugen $(\mathrm{N}=249)$ zur Verfügung standen. Die statistische Auswertung der so gewonnenen Daten wurde mit der Auswertung der primär zur Verfügung stehenden Daten verglichen, um zu überprüfen, ob sich durch die LOCF-Analyse eine genauere (sensitivere) Aussage zur untersuchten Fragestellung ableiten lässt.

Der t-Test wurde angewandt, um signifikante Unterschiede in Mittelwerten zweier Gruppen festzustellen. Für diese statistische Analyse verwendeten wir als Referenz den p-Wert. Dieser wurde mit einem Wert von $\mathrm{p}<0,05$ als Indikator für einen signifikanten Unterschied bezüglich der IOD-Mittelwert-Differenzen angenommen.

Die ANCOVA-Analyse (nalysis of covariance) oder sogenannte Kovarianzanalyse diente dazu, den Einfluss einzelner Parameter auf das Ergebnis statistisch zu bewerten. Zum Beispiel wurden einzelne Einflussfaktoren wie Endotamponade und Größe des Vitrektoms in ihrem Einfluss auf den IOD untersucht.

Die statistische Berechnung der oben genannten Analysen wurde von Herrn Sebastian Pfeiffer aus dem Institut für anwendungsorientierte Forschung und klinische Studien (IFS GmbH) in Göttingen durchgeführt. Die graphische Darstellung der Daten und zusätzliche Subgruppenanalyse erfolgte mit Prism 6 (Version 6.07, Graph Pad, La Jolla, USA).

\subsection{Statistische Analyse}

Im erhobenen Datensatz wurden verschiedene Charakteristika festgelegt. Charakteristika, die nur eine Ausprägung pro Patient betrafen, wurden als Patientencharakteristika definiert:

- Alter bei OP (Berechnung: Differenz von OP-Datum und Geburtstag)

- Geschlecht

- Systemische Steroidtherapie (gleiche Ausprägung über alle Visiten)

Visitenunabhängige Charakteristika, die genau eine Ausprägung pro Auge betrafen, wurden als augenspezifische Daten erfasst:

- Operiert? (ja/nein) $(\mathrm{ja}=$ operiertes Auge; nein = kontralaterales Auge, welches als Kontrollauge verwendet wurde)

- Sphärisches Äquivalent

- Linsenstatus (phak/pseudophak) 
- Diagnose (EG/MF/VMT)

- Vitrektomgröße $(20 \mathrm{G} / 23 \mathrm{G})$

- Endotamponade (C2F6/Luft/SF6/keine)

- Peelingfarbstoff (BB/BP/DB/MB/ICG/TB/ILMB)

- $\quad$ IOD-Baseline = IOD prä-OP

Für das nicht operierte Auge waren die Variablen Diagnose, Vitrektomie, Endotamponade und Peelingfarbstoff jeweils ohne Ausprägung und wurden mit „keine“ (k) gekennzeichnet.

Des Weiteren gab es die Kategorie der visitenabhängigen Daten:

- IOD

- Steroidtropfen (ja/nein)

- Anzahl drucksenkender Augentropfen (AT)

Als Zielgröße des primären und sekundären Endpunktes wurde der Augeninnendruck festgelegt. Nach Power-Kalkulation durch die Abteilung für Medizinische Statistik wurde eine Mindestzahl von $\mathrm{N}=200$ Patienten (jeweils ein Auge pro Patient) angestrebt. Waren pro Patient beide Augen operiert, wurde das Auge mit der längeren Nachbeobachtungszeit gewählt.

\subsubsection{Primärer Endpunkt}

Als primärer Endpunkt wurde die Differenz zwischen dem IOD zum Kontrollzeitpunkt 612 Monate und dem IOD prä-OP festgelegt. Diese wurden für beide Augen miteinander verglichen. Zunächst erfolgte die Auswertung mit den Original-Daten, das heißt ohne Interpolierung fehlender Werte.

Seien $d_{o p}$ für das operierte bzw. $d_{\text {nop }}$ für das nicht-operierte Auge die Differenzen zwischen IOD (<6-12 Monate) und IOD (prä-OP), so ist die Null- bzw. Gegenhypothese:

$$
H_{0}: d_{\text {nop }}-d_{o p}=0<=>H_{0}: d_{\text {nop }}=d_{o p} \text { vs. } H_{1}: d_{\text {nop }}-d_{o p} \neq 0
$$

Aufgrund der hohen Anzahl fehlender Zeitintervalle wurde eine Sensitivitätsanalyse unter Verwendung der LOCF-Methode durchgeführt. 


\subsubsection{Sekundäre Endpunkte}

Wie auch bei der primären Analyse erfolgte eine Auswertung der Original-Daten. Es wurden zwei sekundäre Endpunkte definiert:

\section{Differenz zwischen dem IOD zum Kontrollzeitpunkt 3-6 Monate und IOD prä-OP}

\section{Differenz zwischen dem IOD zum Kontrollzeitpunkt 12-24 Monate und IOD prä-OP}

Aufgrund der hohen Anzahl fehlender Zeitintervalle wurde auch hier eine Sensitivitätsanalyse unter Verwendung der LOCF-Methode durchgeführt.

\subsubsection{Weitere Einflussgrößen - ANCOVA}

Für das operierte Auge wurde für die Differenz zwischen dem IOD von 6-12 Monaten und dem präoperativen IOD der Einfluss der Faktoren Linsenstatus, Vitrektomie, Endotamponade und Geschlecht, sowie den Kovariaten IOD prä-OP, Alter, Sphärisches Äquivalent und Anzahl drucksenkender Augentropfen mit der ANCOVA-Methode bewertet.

In einem weiteren Modell wurden die Daten beider Augen mit den Faktoren operiert (ja/nein), Linsenstatus, Geschlecht und den Kovariaten IOD prä-OP, Alter, Sphärisches Äquivalent und Anzahl drucksenkender Augentropfen untersucht. Auch für die Einflussfaktoren wurde die Auswertung mittels LOCF-Methode wiederholt.

\subsubsection{Subgruppenanalyse}

Die ANCOVA-Analyse der Kovariaten ergab, dass der Einflussfaktor (operiert/nicht operiert) hinsichtlich der Größe des Vitrektoms einen signifikanten Einfluss auf den IOD gehabt haben könnte. Daher wurde dieser in einer weiteren Auswertung untersucht. Hierfür wurden die Operationsverfahren nach $23 \mathrm{G}$ und $20 \mathrm{G}$ aufgeschlüsselt und die Differenzen der Druckunterschiede zwischen prä-OP und post-OP zu den verschiedenen definierten Zeitintervallen verglichen. 


\section{Ergebnisse}

\subsection{Patienten-Charakteristika}

In die Studie wurden 249 Patienten eingeschlossen. Hiervon waren 137 (55\%) weiblich und 112 (45 \%) männlich. Der älteste Patient war zum Zeitpunkt der Vitrektomie 100 Jahre, der jüngste 44 Jahre alt. Das mediane Alter sowohl der Männer als auch der Frauen lag bei 70 Jahren. Der größte Anteil des Patientenkollektivs lag zwischen 65 und 75 Jahren (Mittelwert \pm Standardabweichung).

Tabelle 3: Alter zur OP

\begin{tabular}{|c|c|c|c|c|c|}
\hline Geschlecht & $\mathrm{N}$ & Durchschnitt & Min & Max & SD \\
\hline weiblich & 137 & 70,07 & 54,05 & 100,7 & 6,6 \\
\hline männlich & 112 & 70,01 & 44,59 & 83,97 & 5,9 \\
\hline gesamt & 249 & 70,04 & 44,59 & 100,7 & 6,3 \\
\hline
\end{tabular}

Es zeigte sich eine nahezu ausgewogene Verteilung von linkem und rechtem operiertem Auge. Insgesamt wurden 124 linke Augen (49,8\%) und 125 rechte Augen (50,2 \%) in die Studie aufgenommen.

Tabelle 4: Operiertes Auge

\begin{tabular}{|c|c|c|c|}
\hline Geschlecht & Links & Rechts & Gesamt \\
\hline weiblich & $73(53,3 \%)$ & $64(46,7 \%)$ & $137(55 \%)$ \\
\hline männlich & $51(45,5 \%)$ & $61(54,5 \%)$ & $112(45 \%)$ \\
\hline gesamt & $124(49,8 \%)$ & $125(50,2 \%)$ & $249(100 \%)$ \\
\hline
\end{tabular}

Auch das sphärische Äquivalent (SÄ) des Brechungsfehlers wurde als möglicher Einflussfaktor auf den IOD mit aufgenommen. 
Im Durchschnitt zeigte sich ein SÄ von +0,35 Dioptrien (D) in operierten Augen. Der Minimalwert betrug minus 15 D (starke Myopie), der Maximalwert plus 7 D (starke Hypermetropie).

Tabelle 5: Sphärisches Äquivalent

\begin{tabular}{|c|c|c|c|c|}
\hline Operiertes Auge & $\mathrm{N}$ & Durchschnitt & Min & Max \\
\hline Ja & 249 & 0,35 & -15 & 7 \\
\hline Nein & 249 & 0,45 & -12 & 7,5 \\
\hline
\end{tabular}

Des Weiteren wurde der Linsenstatus präoperativ als möglicher Einflussfaktor auf den IOD erfasst. 231 (92,8\%) operierte Augen waren zum Zeitpunkt der Operation phak (enthielten ihre natürliche Linse) und nur 18 (7,2\%) der eingeschlossenen Augen waren pseudophak (enthielten eine Kunstlinse). Der Linsenstatus änderte sich postoperativ nicht, da dies ein Ausschlusskriterium der Studie war.

Tabelle 6: Linsenstatus

\begin{tabular}{|c|c|}
\hline phak & pseudophak \\
\hline $231(92,8 \%)$ & $18(7,2 \%)$ \\
\hline
\end{tabular}

Der Zielparameter IOD betrug im Durchschnitt 15,67 mmHg in operierten Augen und 15,49 mmHg in nicht-operierten Augen.

Tabelle 7: Augeninnendruck prä-OP

\begin{tabular}{|c|c|c|c|c|}
\hline Operiertes Auge & N & Durchschnitt & Min & Max \\
\hline Ja & 249 & 15,67 & 9 & 24 \\
\hline Nein & 249 & 15,49 & 6 & 23 \\
\hline
\end{tabular}

Unter den Patienten, die in die Studie aufgenommen wurden, hatten 140 (56,2\%) eine epiretinale Gliose, 100 (40,2\%) ein Makulaforamen, 5 (2\%) ein vitreoretinales Traktions- 
syndrom. Vier Patienten $(1,6 \%)$ hatten ein Makulaforamen mit kombinierter epiretinaler Gliose.

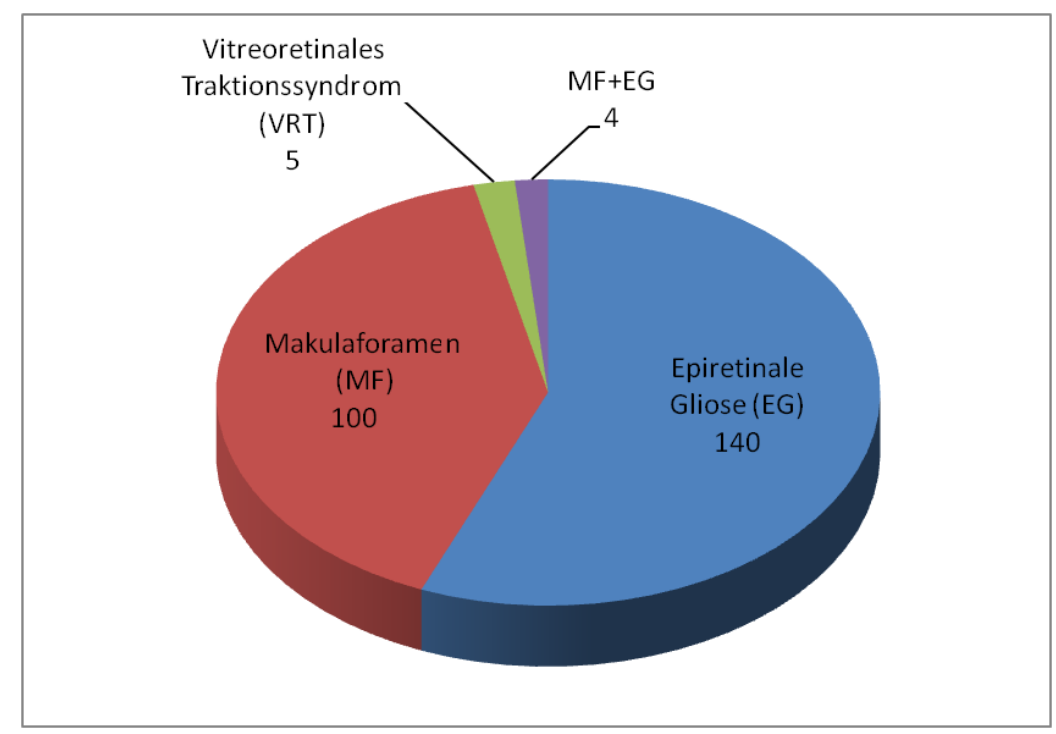

Abbildung 6: Diagnose

\subsection{Operations-Charakteristika}

Für die Vitrektomien wurden Vitrektome der Größe 20 G oder 23 G verwendet. 202 (81,1 \%) der operierten Augen unterzogen sich einer Vitrektomie mit 20 G, $47(18,9 \%)$ mit 23 G.

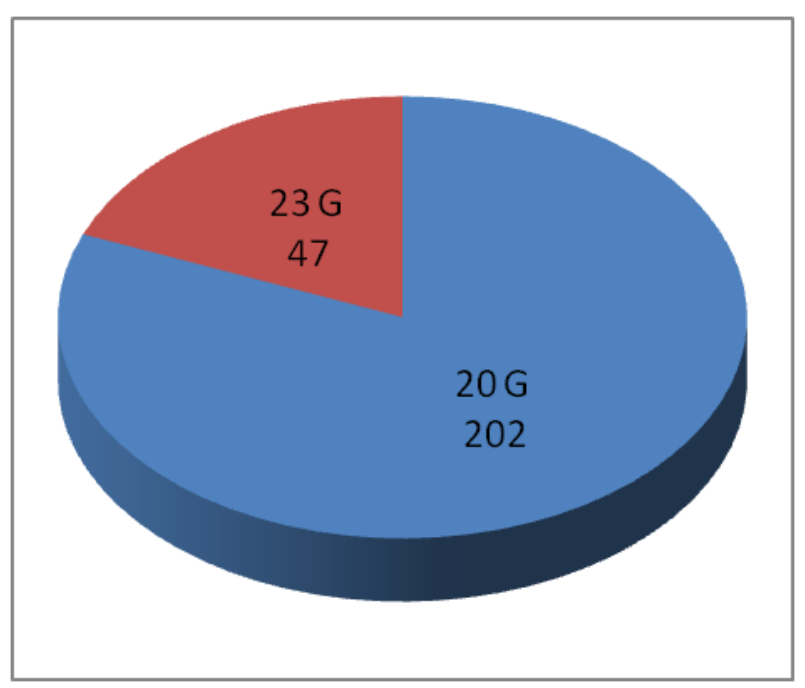

Abbildung 7: Vitrektomie

Nach Vitrektomie wurde der Glaskörper bei 39 (15,7 \%) Patienten mit C2F6, bei 19 (7,6 \%) mit Luft und $73(29,3 \%)$ mit SF6 als Endotamponade gefüllt. 118 der operierten Augen $(47,4 \%)$ erhielten keine Endotamponade. 


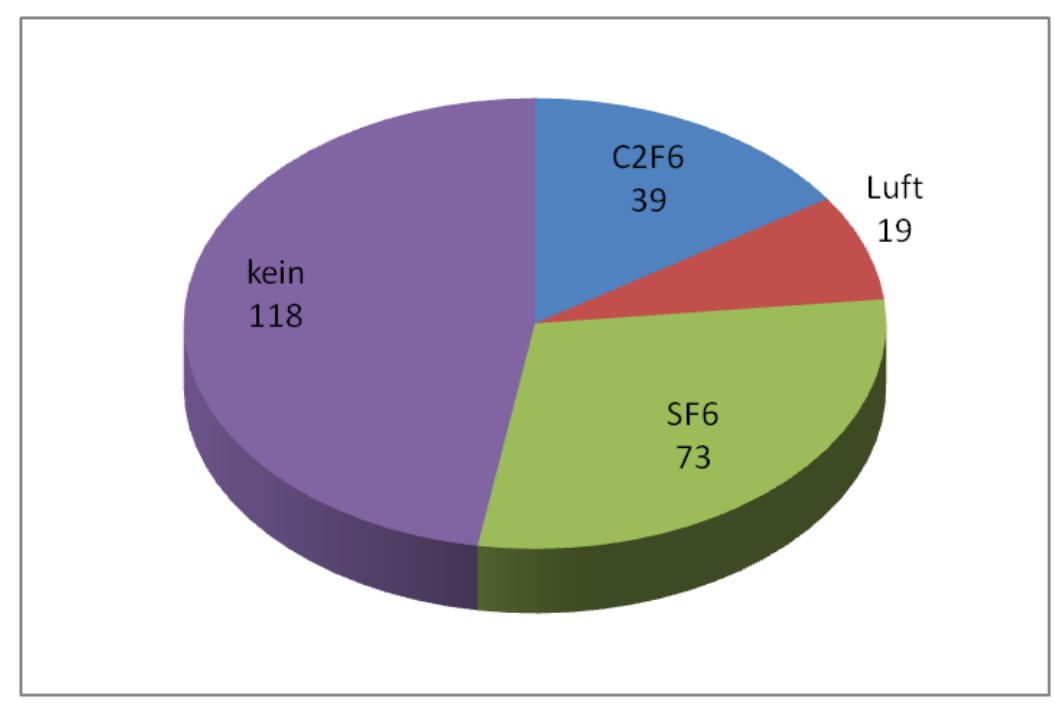

Abbildung 8: Endotamponade

Der intraoperativ eingesetzte Peelingfarbstoff wurde ebenfalls als potentieller Einflussfaktor auf den IOD berücksichtigt. Bei der Mehrzahl der Patienten wurde entweder Brilliant Peel $(103 ; 41,4 \%)$ oder kein Farbstoff $(56 ; 22,5 \%)$ verwendet. Die anderen Farbstoffe mit Brilliant Blau (27; 10,8 \%), Indocyaningrün (23; 9,2 \%), ILM-Blue (22; 8,8 \%), Dual Blue (16; 6,4 \%), Methylenblau (1;0,4 \%) und Trypanblau (1;0,4\%) kamen seltener zur Anwendung.

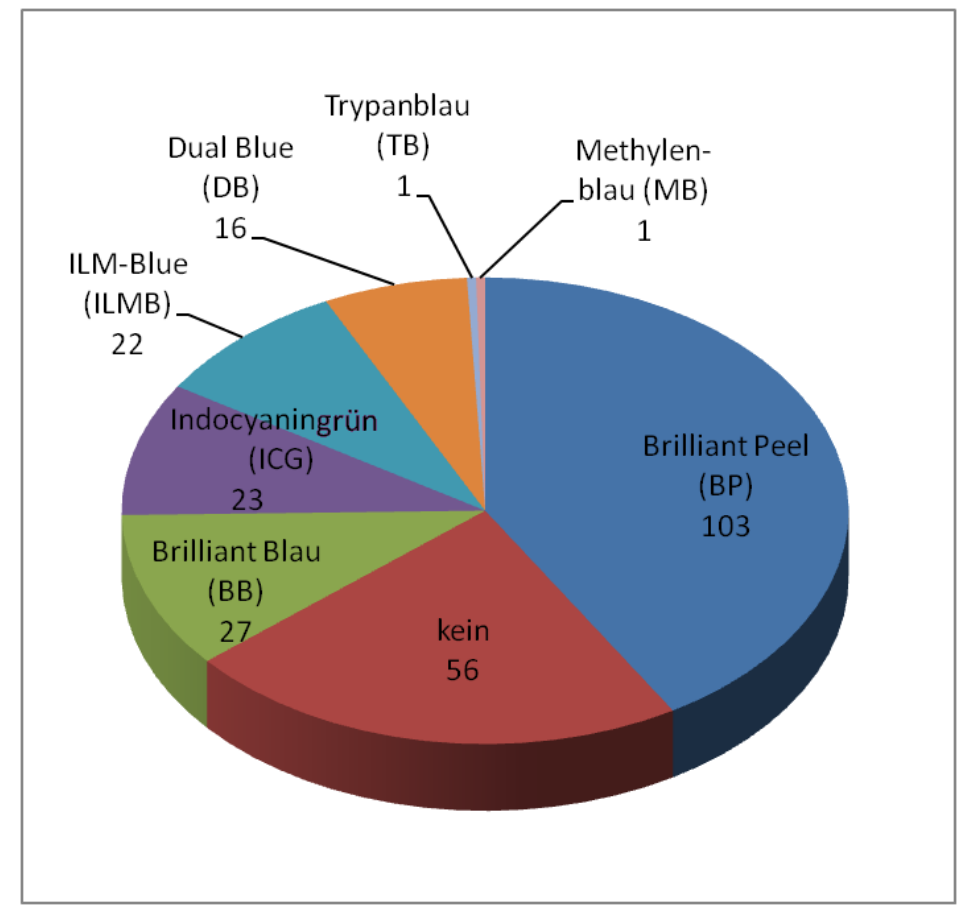

Abbildung 9: Peelingfarbstoff 


\subsection{Statistische Auswertung}

\subsubsection{Augeninnnendruck: Übersicht der Daten}

In Abbildung 10 sind die IOD-Werte aller operierten Augen zu allen erfassten Zeitpunkten in einem Punktwolkendiagramm dargestellt. Der mittlere Balken gibt den Mittelwert an, die Fehlerbalken zeigen die Standardabweichung an

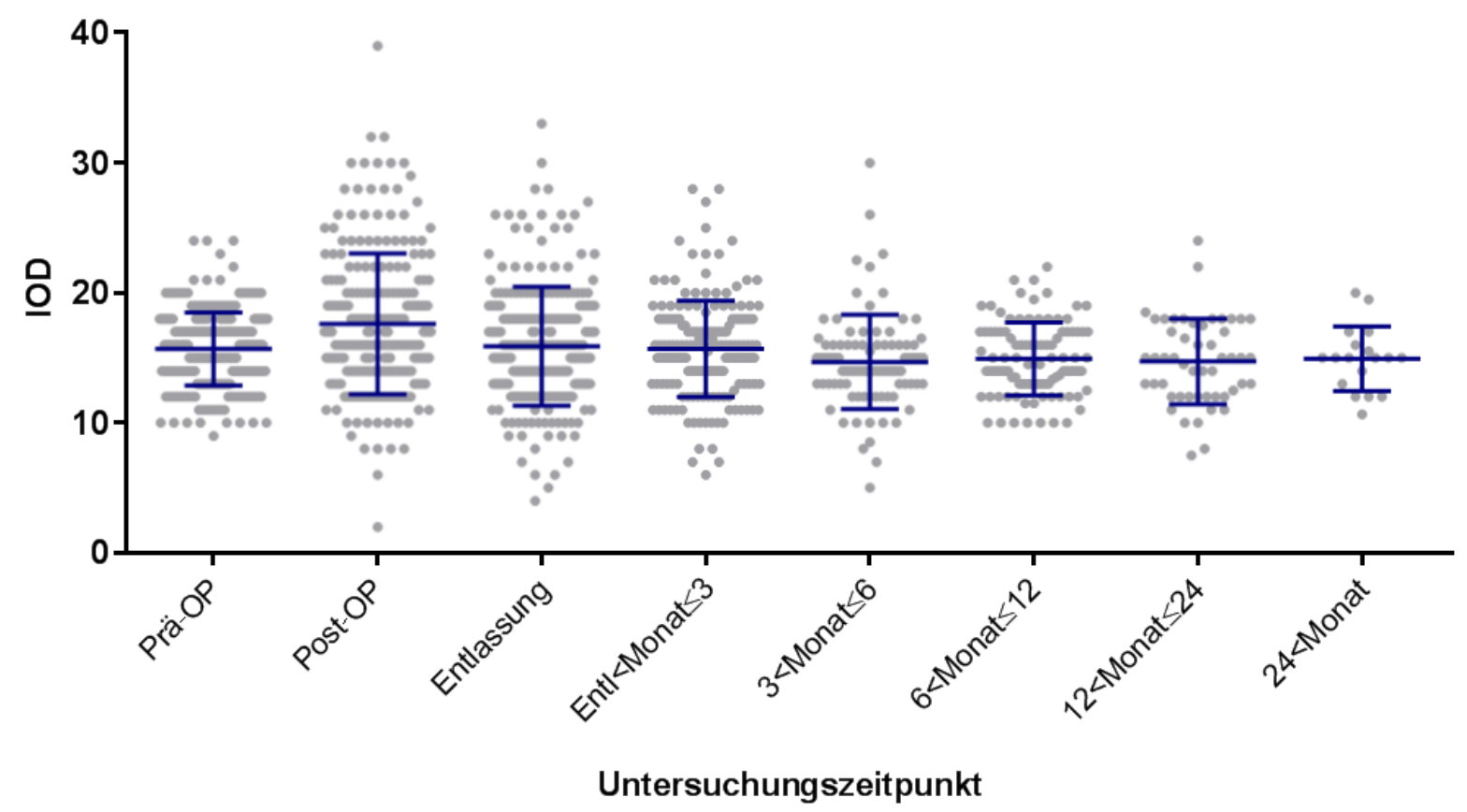

Abbildung 10: Punktwolkendiagramm aller IOD-Werte für das operierte Auge mit Mittelwert und SD

Tabelle 8 zeigt die deskriptive Statistik des IOD zu den verschiedenen Untersuchungszeitpunkten für das operierte Auge. 
Tabelle 8: IOD des operierten Auges zu verschiedenen Zeitpunkten

\begin{tabular}{|c|c|c|c|c|c|c|c|c|c|}
\hline Visite & $\mathrm{N}$ & Mean & $\mathrm{SD}$ & SEM & Min & Median & Max & $\begin{array}{c}\text { Mean } \\
\text { (95\%LCL) }\end{array}$ & $\begin{array}{c}\text { Mean } \\
\text { (95\%UCL) }\end{array}$ \\
\hline prä-OP & 249 & 15,67 & 2,81 & 0,18 & 9 & 16 & 24 & 15,32 & 16,02 \\
\hline post-OP & 225 & 17,6 & 5,42 & 0,36 & 2 & 17 & 39 & 16,89 & 18,32 \\
\hline Entlassung & 244 & 15,88 & 4,56 & 0,29 & 4 & 16 & 33 & 15,3 & 16,45 \\
\hline Ent $\mathrm{l}<$ Monat $\leq \mathbf{3}$ & 184 & 15,68 & 3,70 & 0,27 & 6 & 16 & 28 & 15,14 & 16,22 \\
\hline $3<$ Monat $\leq 6$ & 91 & 14,68 & 3,63 & 0,38 & 5 & 14 & 30 & 13,92 & 15,43 \\
\hline $6<$ Monat $\leq 12$ & 98 & 14,91 & 2,80 & 0,28 & 10 & 14,5 & 22 & 14,35 & 15,48 \\
\hline $12<$ Monat $\leq 24$ & 51 & 14,72 & 3,29 & 0,46 & 7,5 & 15 & 24 & 13,79 & 15,64 \\
\hline $24<$ Monat & 18 & 14,93 & 2,49 & 0,59 & 10,67 & 15 & 20 & 13,69 & 16,16 \\
\hline
\end{tabular}

Um eine erste Tendenz des IOD-Mittelwerts festzustellen, wurden für jedes der definierten Zeitintervalle verbundene t-Tests für das operierte Auge durchgeführt. Für jedes Intervall wurde der präoperative IOD eines Patienten mit dem IOD-Wert des jeweiligen Zeitpunktes verglichen. Da nicht jeder Patient in jedem Zeitintervall ein Messergebnis nachweisen konnte, unterschied sich die Anzahl der Messwerte. In Abbildung 11 wurden die IOD-Mittelwerte prä-OP im jeweiligen Vergleich zum definierten Zeitintervall, deren $\mathrm{N}$ und p-Wert dargestellt. Postoperativ zeigte sich ein signifikanter Druckunterschied zum präoperativen IOD mit einem Anstieg von 1,91 mmHg (p-Wert < 0,001). Nach 6-12 Monaten FU ließ sich bis einschließlich 12-24 Monaten ein signifikanter IOD-Abfall nachvollziehen (6-12 Monate: -0,75 mmHg, p-Wert: 0,008; 12-24 Monate: -1,22 mmHg, p-Wert: 0,007). 


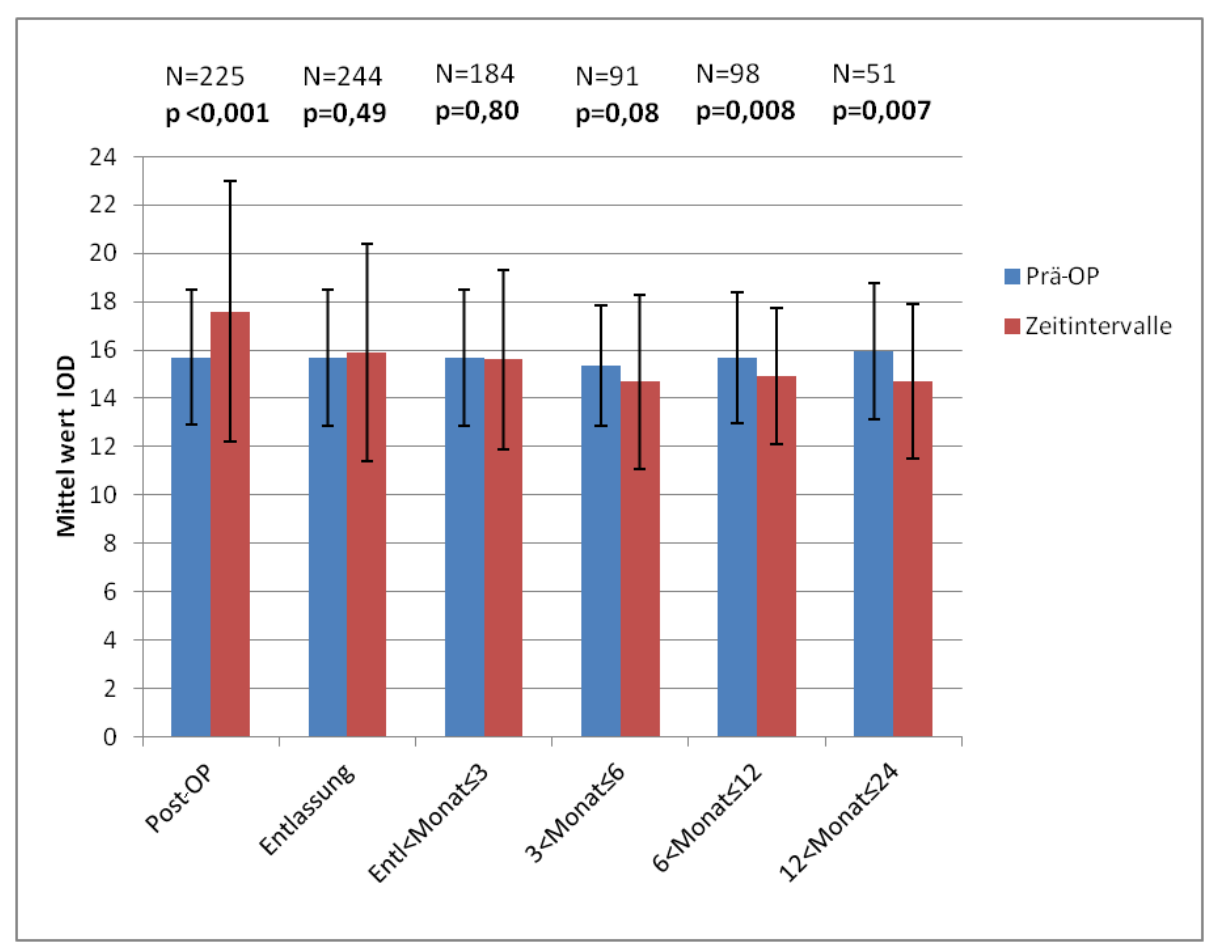

Abbildung 11 : Verbundene t-Tests des IOD von prä-OP zu definierten Zeitintervallen operiertes Auge mit SD

Auch für das nicht operierte Auge wurden alle Werte zu den verschiedenen Zeitpunkten sowohl in einem Punktewolkendiagramm in Abbildung 12 als auch tabellarisch (Tabelle 9) dargestellt.

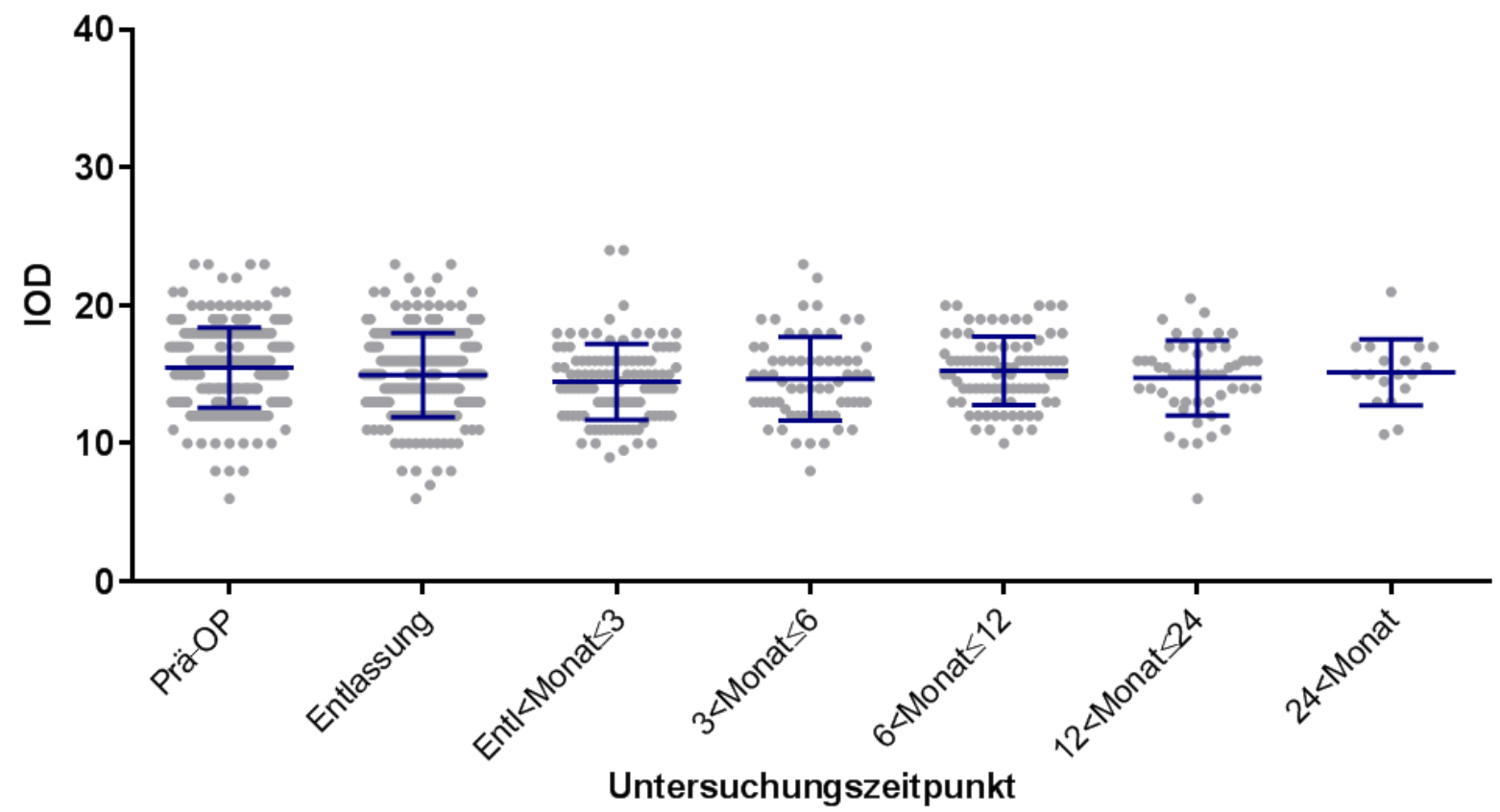

Abbildung 12: Punktwolkendiagramm aller IOD-Werte für das nicht-operierte Auge mit Mittelwert und SD 
Tabelle 9: IOD des nicht-operierten Auges zu verschiedenen Zeitpunkten

\begin{tabular}{|c|c|c|c|c|c|c|c|c|c|}
\hline Visite & $\mathrm{N}$ & Mean & $\mathrm{SD}$ & SEM & Min & Median & Max & $\begin{array}{c}\text { Mean } \\
\text { (95\%LCL) }\end{array}$ & $\begin{array}{c}\text { Mean } \\
\text { (95\%UCL) }\end{array}$ \\
\hline prä-OP & 249 & 15,49 & 2,9 & 0,18 & 6 & 15 & 23 & 15,13 & 15,86 \\
\hline Entlassung & 232 & 14,94 & 3,05 & 0,2 & 6 & 15 & 23 & 14,55 & 15,34 \\
\hline Entl $<$ Monat $\leq 3$ & 104 & 14,46 & 2,76 & 0,27 & 9 & 14 & 24 & 13,9 & 14,99 \\
\hline $3<$ Monat $\leq 6$ & 65 & 14,67 & 3,04 & 0,37 & 8 & 14,5 & 23 & 13,92 & 15,42 \\
\hline $6<$ Monat $\leq 12$ & 84 & 15,25 & 2,49 & 0,27 & 10 & 15,25 & 20 & 14,71 & 15,79 \\
\hline $12<$ Monat $\leq 24$ & 50 & 14,74 & 2,73 & 0,39 & 6 & 15 & 20,5 & 13,96 & 15,51 \\
\hline $24<$ Monat & 18 & 15,15 & 2,4 & 0,57 & 10,67 & 15 & 21 & 13,96 & 16,34 \\
\hline
\end{tabular}

In Abbildung 13 wurden die IOD-Mittelwerte prä-OP im jeweiligen Vergleich zum definierten Zeitintervall, deren $\mathrm{N}$ und p-Wert, diesmal aber für das nicht-operierte Auge, dargestellt. Bei Entlassung sowie nach 12-24 Monaten ließ sich auch hier ein signifikanter IODAbfall nachvollziehen (Entlassung: p-Wert: < 0,001; 12-24 Monate: p-Wert: 0,008).

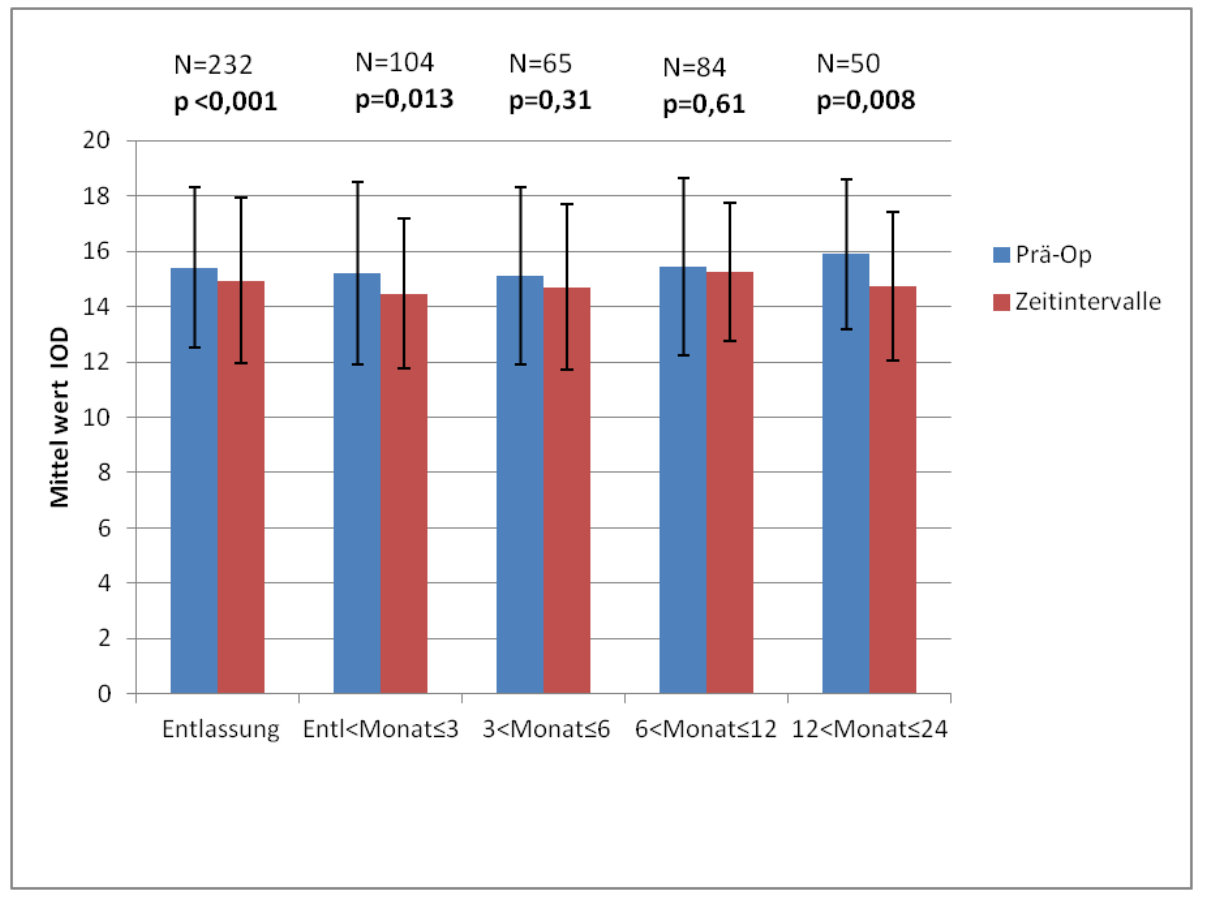

Abbildung 13: Verbundene t-Tests des IOD von prä-OP zu definierten Zeitintervallen nichtoperiertes Auge mit SD 
In Abbildung 14 wurden die Mittelwerte beider Augen zu den unterschiedlichen, vorher definierten Untersuchungszeitpunkten, abgebildet. Zusätzlich wurden die SD sowie die Baseline des IODs mit in die Grafik aufgenommen.

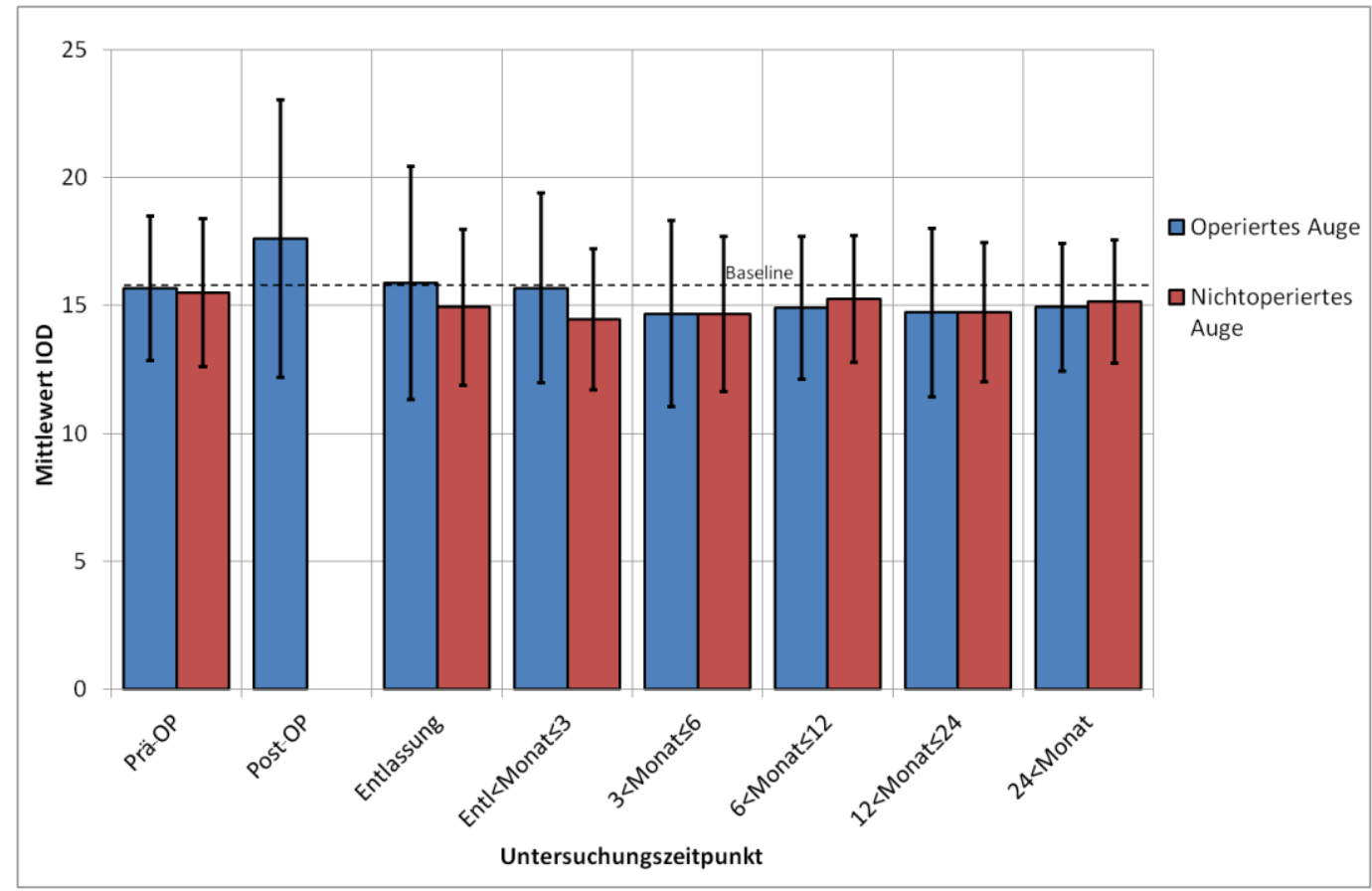

Abbildung 14: Vergleich der IOD-Mittelwerte beider Augen zu unterschiedlichen Untersuchungszeitpunkten mit SD

Beide Ausgangswerte, sowohl der des operierten als auch der des nicht-operierten Auges, lagen auf sehr ähnlichem Niveau. Kurz nach der Operation stieg der Druck des operierten Auges deutlich an, im Durchschnitt um 2 mmHg. (Die Druckwerte des nicht-operierten Auges wurden unmittelbar postoperativ nicht erfasst.) Bei Entlassung lag der IOD aber wieder nahezu auf dem präoperativen Niveau. Im weiteren Verlauf sanken operiertes und nichtoperiertes Auge im Mittel leicht ab.

\subsubsection{Analyse des primären Endpunktes: IOD-Änderung nach 6-12 Monaten}

\subsubsection{Gemessene Daten}

Für alle verfügbaren und gültigen Daten wurde die Differenz zwischen den IOD-Werten vom Zeitintervall 6 bis inklusive 12 Monaten postoperativ und den präoperativen IOD-Werten gebildet:

$$
\text { IOD- } \ddot{\text { Anderung }}=\mathrm{IOD}_{6}<\text { Monat } \leq 12-\mathrm{IOD}_{\text {prä-OP }}
$$


In Abbildung 15 wurde die Verteilung der IOD-Änderung beider Augen dargestellt. Man sieht, dass die Verteilung der Änderungen annähernd einer Gauss'schen Normalverteilung entspricht. Im D'Agostino-Pearson-Test auf Normalverteilung konnte eine statistische Normverteilung nachgewiesen werden.

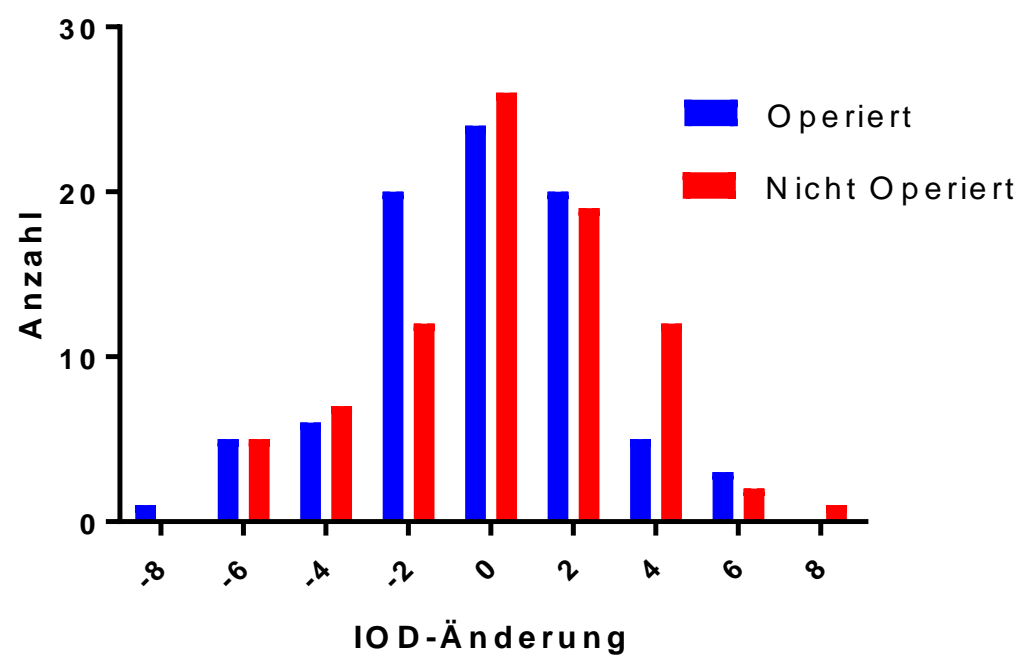

Abbildung 15: Verteilungskurve der IOD-Änderung beider Augen für die primäre Analyse

In Tabelle 10 wurden die Mittelwerte, die Standardabweichung sowie das Konfidenzintervall der IOD-Änderung für beide Augen ermittelt.

Tabelle 10: Statistische Auswertung der IOD-Änderung der primären Analyse

\begin{tabular}{|c|c|c|c|c|c|c|c|}
\hline $\begin{array}{c}\text { Operiertes } \\
\text { Auge }\end{array}$ & $\mathrm{N}$ & Mean & $\mathrm{SD}$ & Min & Max & $\begin{array}{c}\text { Mean } \\
(95 \% \text { LCL })\end{array}$ & $\begin{array}{c}\text { Mean } \\
(95 \% \text { UCL) }\end{array}$ \\
\hline ja & 84 & $-0,66$ & 2,78 & $-8,00$ & 6,00 & $-1,26$ & $-0,06$ \\
\hline nein & 84 & $-0,17$ & 2,98 & $-7,00$ & 7,00 & $-0,81$ & 0,48 \\
\hline
\end{tabular}

In Abbildung 16 wurden alle Differenzen der IOD-Änderung zwischen operiertem und nichtoperiertem Auge in einem Punktdiagramm mit Mittelwert (Mittellinie) und Standardabweichung in Form der Fehlerbalken dargestellt.

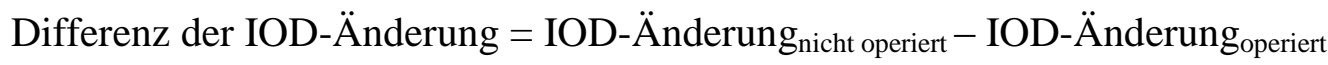




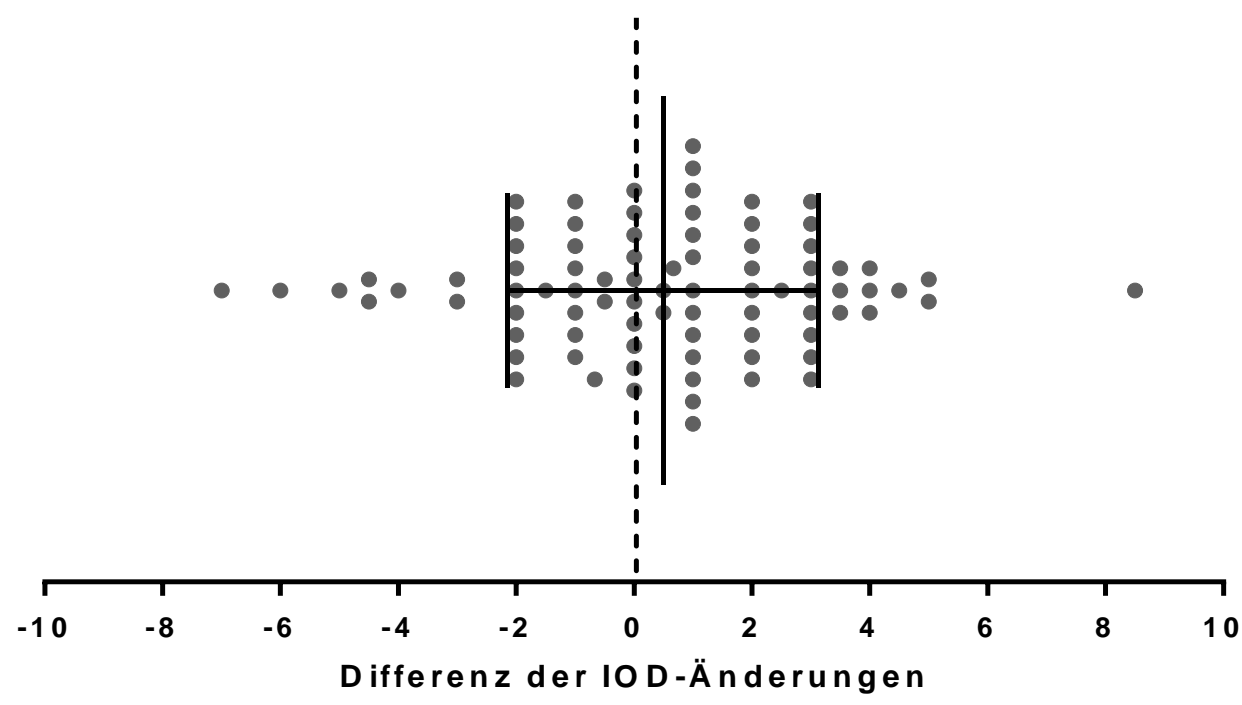

Abbildung 16: Differenz der IOD-Änderung der primären Analyse

Tabelle 11 beschreibt die statistische Auswertung der Differenz der IOD-Änderung zwischen nicht-operiertem und operiertem Auge. Der p-Wert wurde mit Hilfe eines verbundenen tTests aus dem Vergleich der IOD-Änderungen des operierten und nicht-operierten Auges berechnet.

Tabelle 11: Auswertung der Differenz der IOD-Änderung für die primäre Analyse

\begin{tabular}{|c|c|c|c|c|c|}
\hline $\mathrm{N}$ & Mean & SEM & Min & Max & p-Wert \\
\hline $\mathbf{8 4}$ & 0,49 & 0,29 & $-7,00$ & 8,50 & 0,0893 \\
\hline
\end{tabular}

Zusammenfassend zeigte die primäre Analyse, dass die IOD-Differenzen der Werte des Zeitraums 6-12 Monate und den präoperativen Werten einen leichten Abfall des Druckes aufwiesen (-0,66 mmHg für das operierte Auge, $-0,17 \mathrm{mmHg}$ für das nicht-operierte Auge). Im Vergleich der beiden Augen zwischen operiert und nicht-operiert war ein Druckunterschied von ca. 0,5 mmHg zu verzeichnen. Das heißt, die Drucksenkung am operierten Auge war im Mittel um 0,5 mmHg stärker als am nicht-operierten Auge. Der p-Wert war mit 0,0893 größer als 0,05. Das bedeutet, dass diese Differenz nicht ausreichend signifikant war, um die Schlussfolgerung zu ziehen, dass der IOD des operierten Auges tatsächlich stärker abfiel als der des nicht-operierten Auges. Das Ergebnis der Analyse des primären Endpunktes zeigte damit keinen signifikanten Unterschied zwischen den beiden Augen. 


\subsubsection{Sensitivitätsanalyse - LOCF}

Die durchgeführte Sensitivitätsanalyse LOCF (Tabelle 12), welche im Anhang dargestellt wurde, erbrachte keine zusätzlichen Informationen. Hier wurden fehlende Werte zwischen Untersuchungen interpoliert bzw. bei zu kurzer Nachuntersuchung die fehlenden Werte fortgeführt. Der hier ermittelte p-Wert war mit 0,36 größer als 0,05. Das bedeutete, dass auch mit der LOCF-Analyse keine signifikante Änderung der IOD-Werte festgestellt wurde.

Tabelle 12: Statistische Auswertungen des primären Endpunkts mittels LOCF

\begin{tabular}{|c|c|c|c|c|c|c|c|}
\hline $\begin{array}{c}\text { Operiertes } \\
\text { Auge }\end{array}$ & $\mathbf{N}$ & Mean & SD & Min & Max & $\begin{array}{c}\text { Mean } \\
(\mathbf{9 5 \%} \text { LCL) }\end{array}$ & $\begin{array}{c}\text { Mean } \\
(\mathbf{9 5 \%} \text { UCL) }\end{array}$ \\
\hline ja & 249 & $-0,53$ & 3,23 & $-9,00$ & 15,00 & $-0,94$ & $-0,13$ \\
\hline nein & 249 & $-0,35$ & 2,67 & $-9,75$ & 8,00 & $-0,68$ & 0,02 \\
\hline & $\mathbf{N}$ & Mean & SD & Min & Max & t-Wert & p-Wert \\
\hline Differenz & 249 & 0,18 & 3,15 & $-16,00$ & 10,00 & 0,92 & 0,3559 \\
\hline
\end{tabular}

\subsubsection{Korrelation der IOD-Werte prä-OP mit IOD-Werten zwischen 6-12 Monaten}

In Abbildung 17 wurden in einem Streudiagramm die IOD-Werte präoperativ gegen die IODWerte zwischen 6-12 Monate aufgetragen. Zusätzlich wurde eine lineare Regressionsanalyse durchgeführt und die Regressionsgerade in das Diagramm eingefügt.
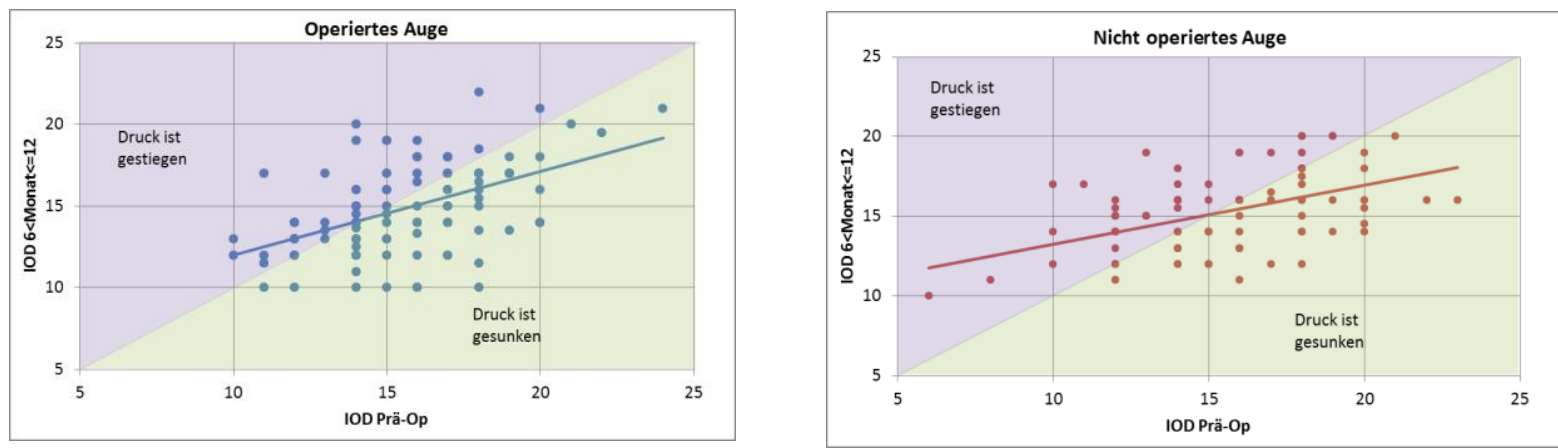

Abbildung 17: Korrelation der IOD-Werte prä-OP mit IOD-Werten zwischen 6-12 Monate 
Je mehr Datenpunkte in der grünen Hälfte lagen, desto mehr Augen zeigten einen IOD-Abfall nach der Operation. Mit Hilfe der Lage und der Steigung der Regressionsgeraden ließen sich diese Veränderungen quantifizieren. Eine Steigung < 1 (flachere Gerade) zeigte eine Drucksenkung bei hohem Ausgangsdruck und eine Drucksteigerung bei niedrigem Ausgangsdruck an. Eine Verschiebung der Geraden nach unten bedeutete eine IOD-Reduktion aller Augen. In der Gruppe der nicht-operierten Augen zeigte sich hauptsächlich eine Abflachung der Geraden. In der Gruppe der operierten Augen fand sich dagegen zusätzlich eine Verschiebung der Geraden nach unten.

\subsubsection{Analyse sekundärer Endpunkte; Teil 1: IOD-Änderung nach 3-6 Monaten}

\subsubsection{Gemessene Daten}

Analog zur Analyse des primären Endpunktes wurde zur Bestimmung der IOD-Änderung die Differenz zwischen den IOD-Werten vom Zeitintervall 3-6 Monate postoperativ und den präoperativen IOD-Werten gebildet:

$$
\mathrm{IOD} \text {-Änderung }=\mathrm{IOD}_{3}<\text { Monat } \leq 6-\mathrm{IOD}_{\text {prä-OP }}
$$

In Abbildung 18 wurde die Verteilung dieser IOD-Änderung beider Augen dargestellt.

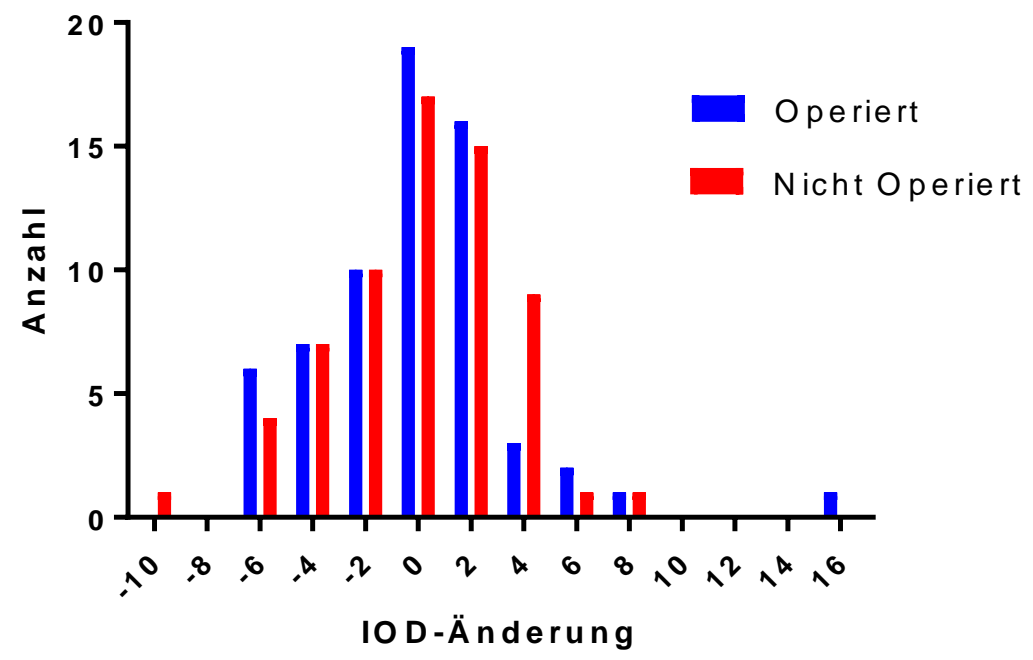

Abbildung 18: Verteilungskurve der IOD-Änderung beider Augen für den sekundären Endpunkt 3-6 Monate 
Tabelle 13: Statistische Auswertung der IOD-Änderung für den sekundären Endpunkt 3-6 Monate

\begin{tabular}{|c|c|c|c|c|c|c|c|}
\hline $\begin{array}{c}\text { Operiertes } \\
\text { Auge }\end{array}$ & $\mathrm{N}$ & Mean & $\mathrm{SD}$ & $\mathrm{Min}$ & $\mathrm{Max}$ & $\begin{array}{c}\text { Mean } \\
(95 \% \mathrm{LCL})\end{array}$ & $\begin{array}{c}\text { Mean } \\
(95 \% \text { UCL })\end{array}$ \\
\hline ja & 65 & $-0,53$ & 3,68 & $-7,00$ & 15,00 & $-1,44$ & 0,38 \\
\hline nein & 65 & $-0,42$ & 3,34 & $-10,00$ & 8,00 & $-1,25$ & 0,41 \\
\hline
\end{tabular}

Tabelle 14: Differenz der IOD-Änderung für den sekundären Endpunkt 3-6 Monate

\begin{tabular}{|c|c|c|c|c|c|}
\hline $\mathrm{N}$ & Mean & SEM & Min & Max & p-Wert \\
\hline $\mathbf{6 5}$ & 0,11 & 0,45 & $-16,00$ & 9,00 & 0,81 \\
\hline
\end{tabular}

Der erste Teil der sekundären Endpunktanalyse zeigte, dass die IOD-Änderung am operierten Auge nach 3-6 Monaten einen leichten Druckabfall ergibt $(-0,53 \pm 3,68 \mathrm{mmHg}$; Tabelle 13). Jedoch trat auch bei den nicht-operierten Augen eine leichte Drucksenkung auf (-0,42 \pm 3,34 mmHg; Tabelle 13). Nach Berechnung der Differenz zwischen operiertem und nichtoperiertem Auge zeigte sich kein signifikanter Unterschied zwischen den IOD-Änderungen beider Augen ( $\mathrm{p}=0,81$, Tabelle 14).

\subsubsection{Sensitivitätsanalyse - LOCF}

Die durchgeführte Sensitivitätsanalyse LOCF (Tabelle 15) erbrachte auch für diese Analyse keine zusätzlichen Informationen. Der Unterschied zwischen operiertem und nicht-operiertem Auge blieb statistisch nicht signifikant $(\mathrm{p}=0,72)$. 
Tabelle 15: Statistische Auswertungen des sekundären Endpunkts mittels LOCF für den Zeitraum 3-6 Monate

\begin{tabular}{|c|c|c|c|c|c|c|c|}
\hline $\begin{array}{c}\text { Operiertes } \\
\text { Auge }\end{array}$ & $\mathbf{N}$ & Mean & SD & Min & Max & $\begin{array}{c}\text { Mean } \\
(\mathbf{9 5 \%} \text { LCL) }\end{array}$ & $\begin{array}{c}\text { Mean } \\
(\mathbf{9 5 \%} \text { UCL) }\end{array}$ \\
\hline ja & 249 & $-0,28$ & 3,23 & $-9,00$ & 15,00 & $-0,68$ & $-0,12$ \\
\hline nein & 249 & $-0,35$ & 2,49 & $-10,00$ & 8,00 & $-0,66$ & 0,04 \\
\hline & $\mathbf{N}$ & Mean & SD & Min & Max & t-Wert & p-Wert \\
\hline Differenz & 249 & 0,07 & 3,10 & $-16,00$ & 9,00 & $-0,36$ & 0,72 \\
\hline
\end{tabular}

\subsubsection{Analyse sekundärer Endpunkte; Teil 2: IOD-Änderung nach 12-24 Monaten}

\subsubsection{Gemessene Daten}

Für diese Analyse wurde die IOD-Änderung im Zeitintervall 12-24 Monate berechnet:

$$
\mathrm{IOD} \text { - Änderung }=\mathrm{IOD}_{12}<\text { Monat } \leq 24-\mathrm{IOD}_{\text {prä-OP }}
$$

In Abbildung 19 wurde die Verteilung dieser IOD-Änderungen beider Augen dargestellt.

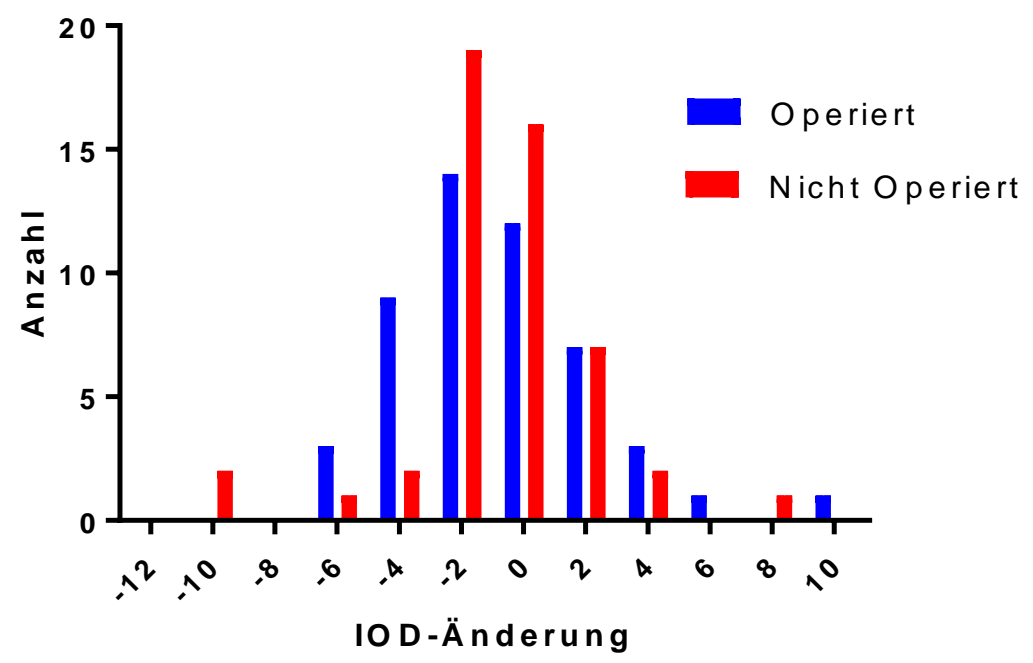

Abbildung 19: Verteilungskurve der IOD-Änderungen beider Augen für den sekundären Endpunkt 12-24 Monate 
Tabelle 16: Statistische Auswertung der IOD-Differenz, sekundärer Endpunkt 12 bis 24 Monate

\begin{tabular}{|c|c|c|c|c|c|c|c|}
\hline $\begin{array}{c}\text { Operiertes } \\
\text { Auge }\end{array}$ & $\mathrm{N}$ & Mean & $\mathrm{SD}$ & $\mathrm{Min}$ & $\mathrm{Max}$ & $\begin{array}{c}\text { Mean } \\
(95 \% \text { LCL })\end{array}$ & $\begin{array}{c}\text { Mean } \\
(95 \% \text { UCL })\end{array}$ \\
\hline ja & 50 & $-1,21$ & 3,13 & $-7,00$ & 10,00 & $-2,09$ & $-0,32$ \\
\hline nein & 50 & $-1,16$ & 2,96 & $-11,00$ & 7,00 & $-2,01$ & $-0,32$ \\
\hline
\end{tabular}

Tabelle 17: Differenz der IOD-Änderung, sekundärer Endpunkt 12-24 Monate

\begin{tabular}{|c|c|c|c|c|c|}
\hline $\mathrm{N}$ & Mean & SEM & Min & Max & p-Wert \\
\hline $\mathbf{5 0}$ & 0,04 & 0,45 & $-9,00$ & 11,00 & 0,9231 \\
\hline
\end{tabular}

Wie auch im ersten Teil der Analyse kam es am operierten Auge zu einer Drucksenkung postoperativ $(-1,21 \pm 3,13 \mathrm{mmHg}$, Tabelle 16). Allerdings trat auch hier am nicht-operierten Auge ebenfalls eine Drucksenkung auf $(-1,16 \pm 2,96 \mathrm{mmHg}$, Tabelle 16), so dass die Differenz der Änderung statistisch nicht signifikant war ( $p=0,92$, Tabelle 17).

\subsubsection{Sensitivitätsanalyse - LOCF}

Die durchgeführte Sensitivitätsanalyse LOCF (Tabelle 18) erbrachte auch für diese Analyse keine zusätzlichen Informationen. Die Differenz der IOD-Änderung blieb statistisch nicht signifikant $(\mathrm{p}=0,31)$.

Tabelle 18: Statistische Auswertungen des sekundären Endpunkts mittels LOCF für den Zeitraum 12-24 Monate

\begin{tabular}{|c|c|c|c|c|c|c|c|}
\hline $\begin{array}{c}\text { Operiertes } \\
\text { Auge }\end{array}$ & $\mathbf{N}$ & Mean & SD & Min & Max & $\begin{array}{c}\text { Mean } \\
(\mathbf{9 5 \%} \text { LCL) }\end{array}$ & $\begin{array}{c}\text { Mean } \\
(\mathbf{9 5 \%} \text { UCL) }\end{array}$ \\
\hline ja & 249 & $-0,63$ & 3,26 & $-9,00$ & 15,00 & $-1,04$ & $-0,22$ \\
\hline nein & 249 & $-0,42$ & 2,42 & $-11,00$ & 8,00 & $-0,76$ & 0,08 \\
\hline & $\mathbf{N}$ & Mean & SD & Min & Max & t-Wert & p-Wert \\
\hline Differenz & 249 & 0,21 & 3,25 & $-16,00$ & 11,00 & 1,01 & 0,3145 \\
\hline
\end{tabular}




\subsubsection{Zusammenfassung der drei Analysen}

In Abbildung 20 wurden zusammenfassend die Mittelwerte der IOD-Differenzen zu allen untersuchten Zeitpunkten dargestellt. Die Fehlerbalken geben den Standardfehler des Mittelwerts an.

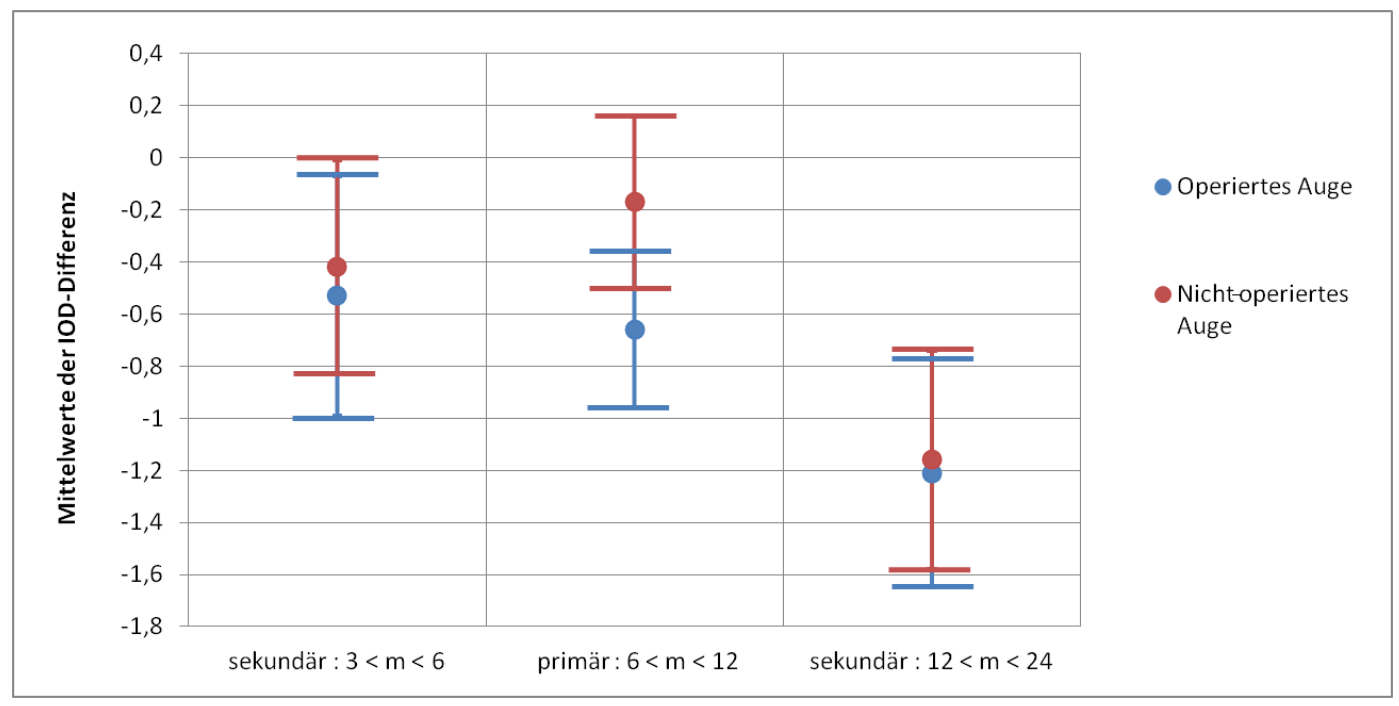

Abbildung 20: Mittelwerte der IOD-Änderungen für die drei Analysen beider Augen

Die Darstellung zeigte, dass die Mittelwerte der IOD-Änderungen relativ gering waren und alle in negative Richtung (Drucksenkung) tendierten. Operiertes und nicht-operiertes Auge änderten sich stets gleichsinnig. Der Mittelwert des operierten Auges lag hierbei immer niedriger als der des nicht-operierten Auges. Gemessen an der relativ großen Streuung der Werte war jedoch diese Differenz zu keinem Zeitintervall statistisch signifikant.

\subsubsection{Einflussfaktoren auf den IOD über ANCOVA-Analyse}

Die untersuchten Patienten hatten unterschiedliche Eigenschaften (Linsenstatus, Geschlecht) und wurden mit unterschiedlichen Operationstechniken (z. B. Endotamponade, Vitrektomgröße) operiert.

Mittels ANCOVA-Analyse untersuchten wir die in Tabelle 19 zusammengefassten Faktoren auf ihren möglichen Einfluss auf die IOD-Änderung. Hierfür wurde die IOD-Änderung zum Zeitpunkt des primären Endpunktes (6-12 Monate) verwendet. 
Tabelle 19: Einteilung der Einflussfaktoren

\begin{tabular}{|l|c|l|}
\hline Einflussfaktor & Anzahl der Parameter & \multicolumn{1}{|c|}{ Parameter } \\
\hline Linsenstatus & 2 & $\begin{array}{l}\text { phak } \\
\text { pseudophak }\end{array}$ \\
\hline Vitrektomgröße & 2 & $\begin{array}{l}20 \mathrm{G} \\
23 \mathrm{G}\end{array}$ \\
\hline Endotamponade & 4 & $\begin{array}{l}\text { C2F6 } \\
\text { Luft } \\
\text { SF6 } \\
\text { k }\end{array}$ \\
\hline Geschlecht & 2 & $\begin{array}{l}\text { männlich } \\
\text { weiblich }\end{array}$ \\
\hline Alter & - & je nach Geburtsdatum \\
\hline SÄ & - & $\begin{array}{l}\text { Wert des S ̈̈ } \\
\text { Nein }\end{array}$ \\
\hline N drucksenkende AT & 2 & $\begin{array}{l}\text { Ja } \\
\text { Nein }\end{array}$ \\
\hline Operiert & 2 & \\
\hline
\end{tabular}

In Tabelle 20 wurde die Signifikanz des Einflusses der untersuchten Faktoren auf den IOD nach ANCOVA-Analyse dargestellt.

Tabelle 20: Signifikanz der Einflussfaktoren

\begin{tabular}{|l|c|c|}
\hline Einflussfaktor & $\mathrm{N}$ & $\mathrm{p}$-Wert \\
\hline Linsenstatus & 87 & 0,6 \\
\hline Vitrektomgröße & 87 & 0,043 \\
\hline Endotamponade & 87 & 0,498 \\
\hline Geschlecht & 87 & 0,414 \\
\hline Alter & 87 & 0,877 \\
\hline Sphärisches Äquiv. & 87 & 0,977 \\
\hline N drucksenkende AT & 87 & 0,815 \\
\hline Operiert & 174 (beide & 0,196 \\
\hline
\end{tabular}


Man sah, dass Linsenstatus (phak/pseudophak), verschiedene Endotamponaden (SF6, C2F6, Luft, keine), das Geschlecht, das Alter, das sphärische Äquivalent sowie die Anzahl der drucksenkenden Augentropfen keinen signifikanten Einfluss auf die IOD-Änderung nahmen (p-Wert > 0,05). Zu sehen war jedoch auch, dass der p-Wert des Einflussfaktors „Vitrektomgröße“ (20 G oder $23 \mathrm{G})$, mit 0,0436 unter 0,05 lag und damit eine statistisch signifikante Einflussgröße darstellte.

Für den Einflussfaktor „operiert“ war der p-Wert mit 0,1963>0,05, was bedeutete, dass eine dezente Tendenz, aber kein signifikanter Unterschied in der IOD-Änderung zwischen operiertem und nicht-operiertem Auge bestand. Dies war in Übereinstimmung mit der Analyse des primären Endpunktes.

\subsubsection{Einflussfaktoren auf den IOD über ANCOVA-Analyse mittels LOCF}

Die ANCOVA-Analyse mit den interpolierten und fortgeschriebenen Werten der LOCFMethode ergab keine auswertbaren Ergebnisse, da zu viele Werte fehlten und dadurch zu viele interpoliert werden mussten. Hiernach wäre kein Einfluss zu erkennen. 


\subsubsection{Subgruppen-Analysen}

\subsubsection{Einfluss der Vitrektomgröße auf den IOD des operierten Auges}

Da die Größe des Vitrektoms in der ANCOVA als Einflussfaktor auf den IOD identifiziert wurde, führten wir eine zusätzliche statistische Auswertung durch, in der die 20-G- und 23-GVitrektomie gegenübergestellt wurden.

In Abbildung 21 wurden alle IOD-Änderungen des operierten Auges zu den definierten Zeitpunkten in $23 \mathrm{G}$ und $20 \mathrm{G}$ eingeteilt und in Punktwolkendiagrammen mit jeweiligem Mittelwert (Mittelbalken) und deren Standardabweichung als Fehlerbalken dargestellt. Hier zeigten sich alle IOD-Änderungen normverteilt, außer im Intervall von 12-24 Monaten der 23-GOperation, wo zu wenige Datenpunkte zur Verfügung standen. Ein Datenpunkt des Zeitraums 3-6 Monate fiel weit aus dem Rahmen (31 mmHg operiertes Auge, $15 \mathrm{mmHg}$ nicht-operiertes Auge) und wurde für die Analyse ausgeklammert, um die Daten nicht zu verzerren. Während für die 20-G-Operation die Mittelwerte sanken, blieben sie für die 23-G-Operationen etwa auf demselben Niveau wie präoperativ. Für den Zeitraum 12-24 Monate war die Schlussfolgerung aufgrund der relativ geringen Gruppengröße von $\mathrm{N}=6$ (Tabelle 21) allerdings nur eingeschränkt verwertbar.

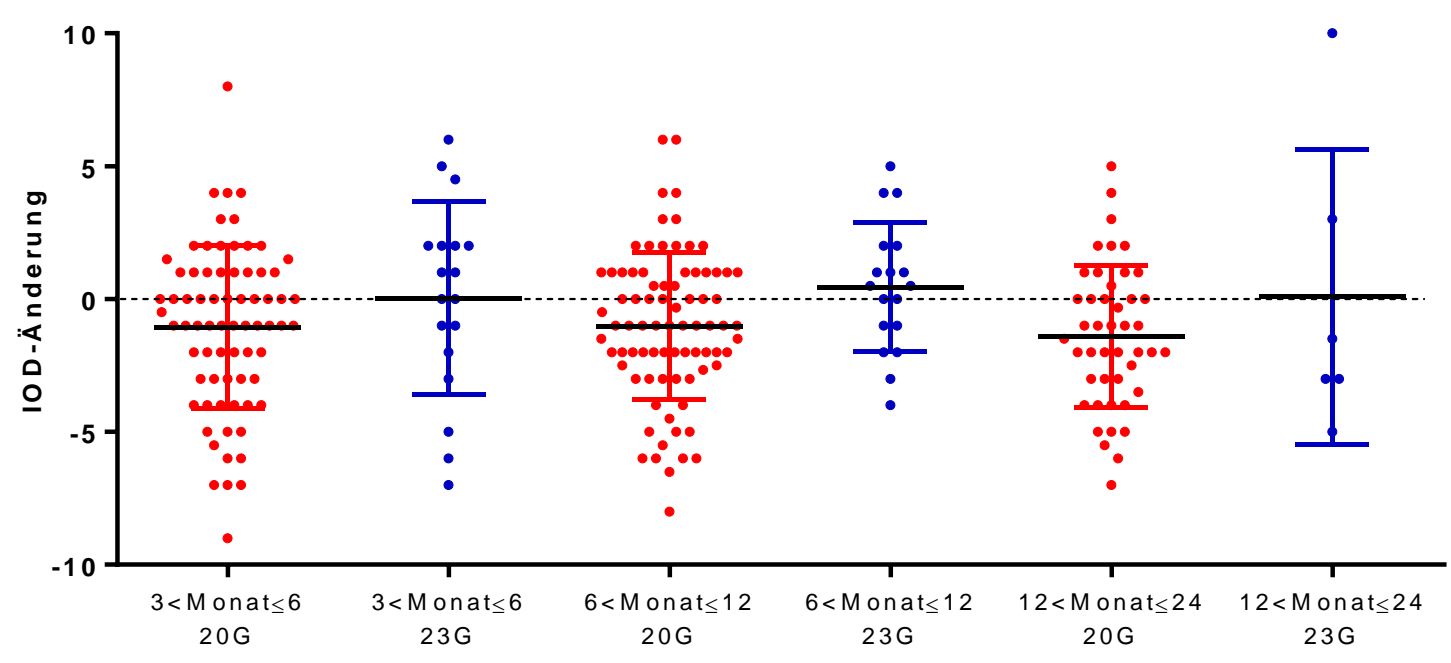

Abbildung 21: IOD-Änderung für jeweils 20 G und 23 G des operierten Auges 
Tabelle 21: Statistische Auswertung der IOD-Änderung des operierten Auges

\begin{tabular}{|c|c|c|c|c|c|c|c|c|}
\hline Zeitpunkt & Vitrektomie & $\mathrm{N}$ & Mean & SD & Min & Max & $\begin{array}{c}\text { Mean } \\
(95 \% \text { LCL })\end{array}$ & $\begin{array}{c}\text { Mean } \\
(95 \% \text { UCL })\end{array}$ \\
\hline $\mathbf{3}<$ Monat $\leq \mathbf{6}$ & $20 \mathrm{G}$ & 72 & $-1,06$ & 3,07 & $-9,00$ & 8,00 & $-1,77$ & $-0,33$ \\
\hline $\mathbf{3}<$ Monat $\leq \mathbf{6}$ & $23 \mathrm{G}$ & 18 & 0,03 & 3,63 & $-7,00$ & 6,00 & $-1,78$ & 1,8 \\
\hline $\mathbf{6}<$ Monat $\leq \mathbf{1 2}$ & $20 \mathrm{G}$ & 80 & $-1,02$ & 2,78 & $-8,00$ & 6,00 & $-1,64$ & $-0,40$ \\
\hline $\mathbf{6}<$ Monat $\leq \mathbf{1 2}$ & $23 \mathrm{G}$ & 18 & 0,44 & 2,42 & $-4,00$ & 5,00 & $-0,76$ & 1,65 \\
\hline $\mathbf{1 2}<$ Monat $\leq \mathbf{2 4}$ & $20 \mathrm{G}$ & 45 & $-1,39$ & 2,67 & $-7,00$ & 5,00 & $-2,19$ & $-0,59$ \\
\hline $\mathbf{1 2}<$ Monat $\leq \mathbf{2 4}$ & $23 \mathrm{G}$ & 6 & 0,08 & 5,55 & $-5,00$ & 10,00 & $-5,74$ & 5,91 \\
\hline
\end{tabular}

Über den t-Test wurde die Differenz zu jedem definierten Zeitintervall im Vergleich der 20G- zur 23-G-Operation berechnet. Hier ergab sich ein signifikanter t-Test mit 0,041 für den Zeitraum des primären Endpunkts (6-12 Monate). Dies wurde in Abbildung 22 dargestellt.

Hieraus konnte geschlussfolgert werden, dass es nach 6-12 Monaten zu einem signifikanten Absinken des IODs unter Vitrektomie mit 20 Gauge kam. Dies müsste in einer separaten Studie weiter untersucht und ggf. bestätigt werden.

Die sekundäre Analyse (3-6 und 12-24 Monate) ergab mathematisch keinen signifikanten pWert, obwohl die Mittelwerte der IOD-Differenzen (20 G: -1,04 mmHg; 23 G: 0,04 mmHg) einen Unterschied aufweisen (Abbildung 22).

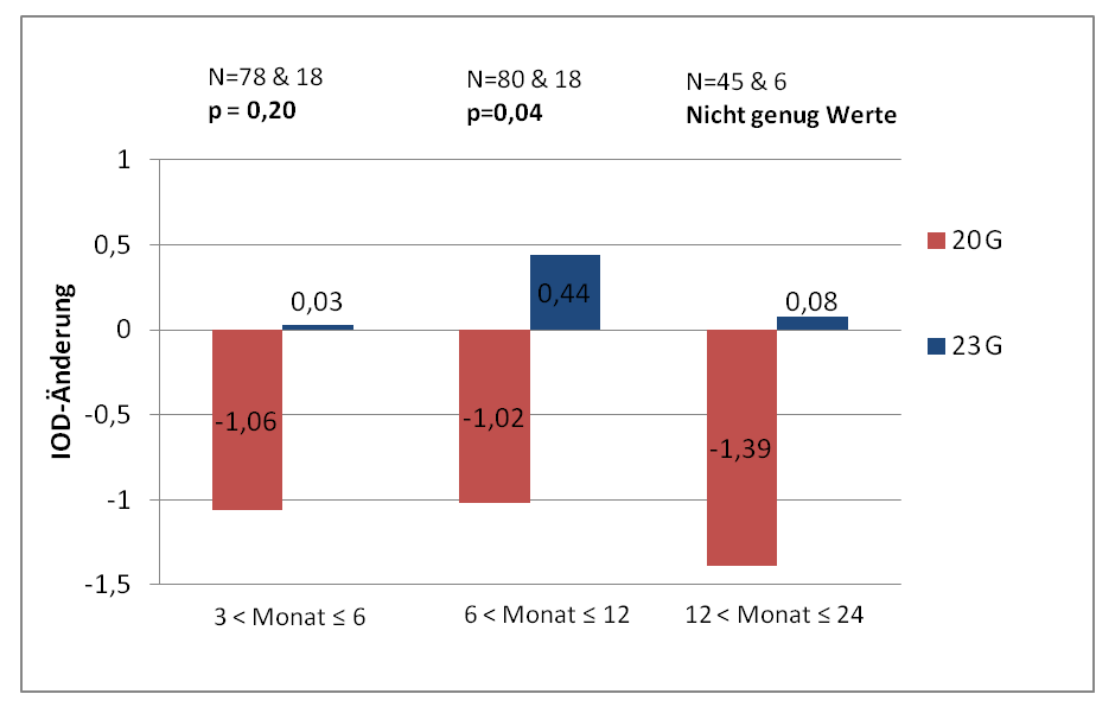

Abbildung 22: Unverbundene t-Tests für die Vitrektomgröße des operierten Auges 
Zur Visualisierung der Änderung der IOD-Werte wurden in Abbildung 23 die IOD-Werte präoperativ gegen die IOD-Werte zu den Zeiträumen 3-6 Monate und 6-12 Monate, jeweils für 20 G (linke Spalte) und 23 G (rechte Spalte) aufgetragen. Zusätzlich wurde eine Regressionsgerade eingefügt.

Auch hier zeigte sich, dass nach 20-G-Operation der Augeninnendruck eher absank, nach 23G-Operation im Durchschnitt unverändert blieb.
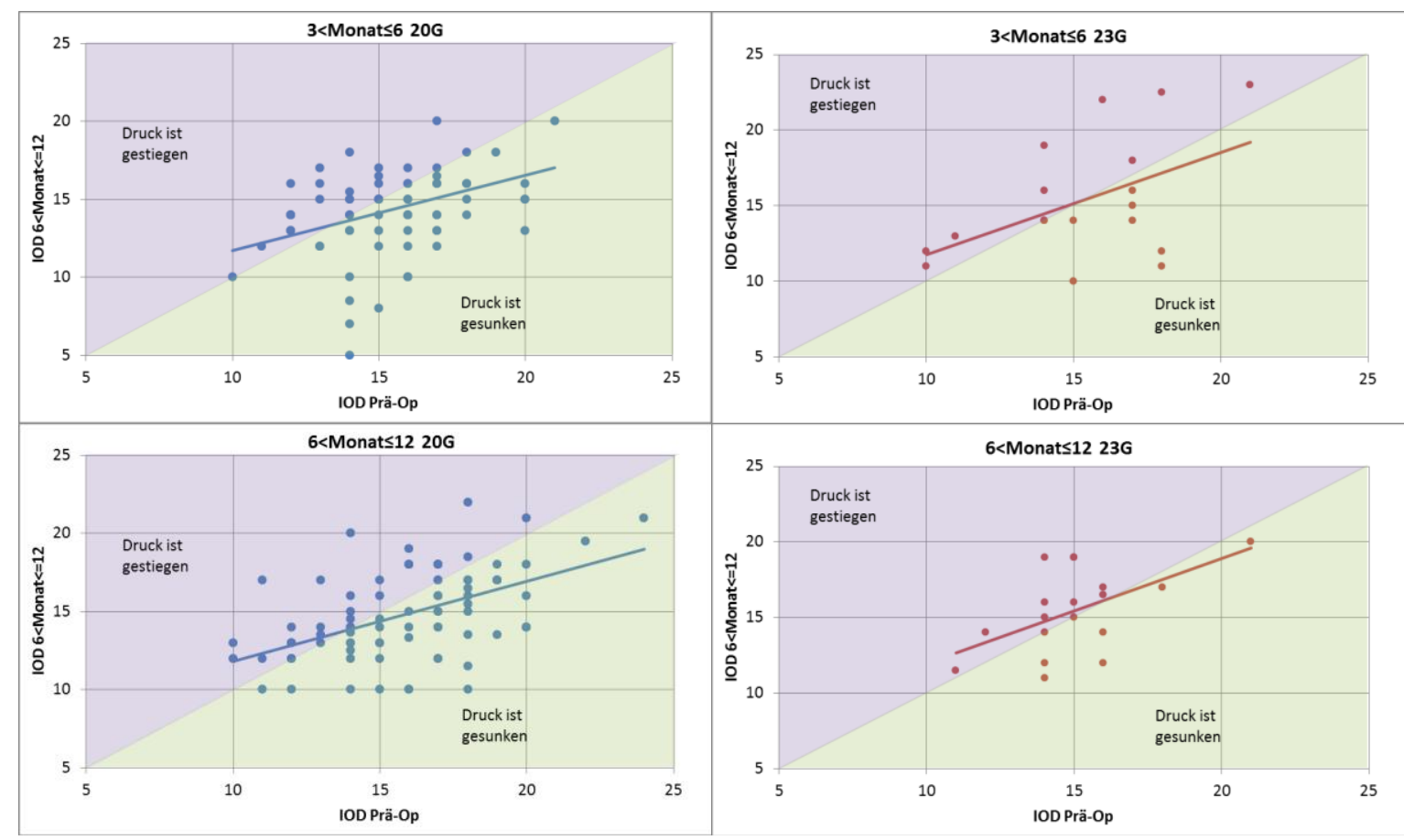

\section{Abbildung 23: Korrelation der IOD-Werte zwischen präoperativen und anderen definierten Zeitintervallen für 20 G- und 23 G-operierte Augen}

\subsubsection{Einfluss der Vitrektomgröße auf den IOD des nicht-operierten Auges}

Bereits in den globalen Analysen der Daten hat sich ein Absinken des IOD nach Vitrektomie am operierten Auge gezeigt. Da aber der IOD am nicht-operierten Auge ebenfalls leicht gesunken war, konnte die beobachtete Druckänderung nicht eindeutig dem Effekt der Vitrektomie zugeschrieben werden. Um dies auch für die Subgruppenanalyse der Vitrektomgröße zu überprüfen, wurde auch für das nicht-operierte Auge die IOD-Änderung berechnet.

In Abbildung 24 wurden, wie auch bereits für das operierte Auge, die Werte zu den definierten Zeitpunkten in 23-G- (blau) und 20-G-Vitrektomie (rot) eingeteilt und in Punktwolkendiagrammen mit jeweiligem Mittelwert (Mittelbalken) und Standardabweichung (Fehlerbalken) dargestellt. Auch beim nicht-operierten Auge waren die Werte normverteilt. 
Für die Zeiträume 3-6 und 6-12 Monate war eine minimale Tendenz zum Absinken des Mittelwertes bei 20-G-Vitrektomie zu erkennen. Die 23-G-Werte blieben bis 12 Monate eher unverändert. Im Zeitraum 12-24 Monate war in beiden Gruppen (20 G und $23 \mathrm{G}$ ) eine negative IOD-Änderung, das heißt eine Drucksenkung zu erkennen, allerdings mit kleiner Gruppengröße, vor allem für die 23-G-Gruppe.

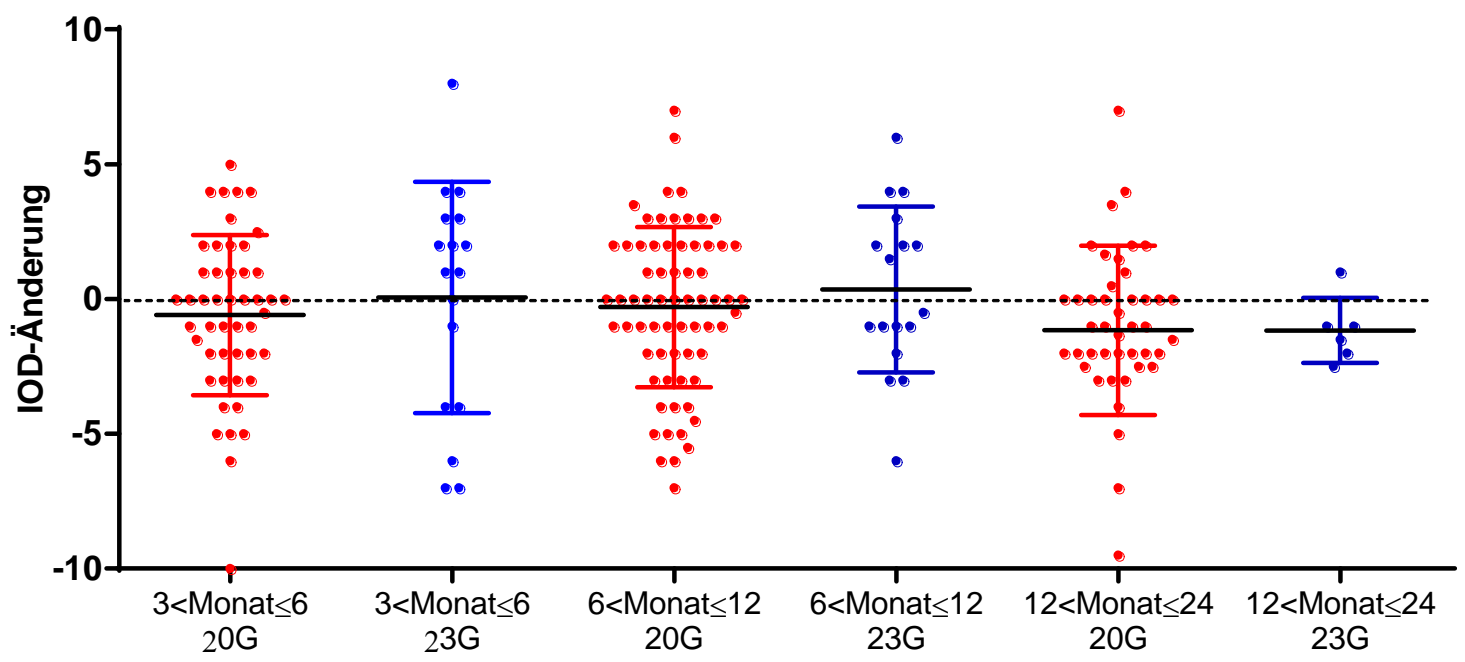

Abbildung 24: IOD-Änderung für jeweils 20 G und 23 G des nicht-operierten Auges

Tabelle 22: Statistische Auswertung der IOD-Änderung des nicht-operierten Auges

\begin{tabular}{|c|c|c|c|c|c|c|c|c|}
\hline Zeitpunkt & Vitrektomie & $\mathrm{N}$ & Mean & SD & Min & Max & $\begin{array}{c}\text { Mean } \\
(95 \% \text { LC })\end{array}$ & $\begin{array}{c}\text { Mean } \\
(95 \% \text { UCL })\end{array}$ \\
\hline $\mathbf{3}<$ Monat $\leq 6$ & $20 \mathrm{G}$ & 48 & $-0,59$ & 2,97 & $-10,00$ & 5,00 & $-1,46$ & 0,27 \\
\hline $\mathbf{3}<$ Monat $\leq \mathbf{6}$ & $23 \mathrm{G}$ & 17 & 0,06 & 4,29 & $-7,00$ & 8,00 & $-2,15$ & 2,26 \\
\hline $\mathbf{6}<$ Monat $\leq \mathbf{1 2}$ & $20 \mathrm{G}$ & 67 & $-0,29$ & 2,97 & $-7,00$ & 7,00 & $-1,02$ & 0,42 \\
\hline $\mathbf{6}<$ Monat $\leq \mathbf{1 2}$ & $23 \mathrm{G}$ & 17 & 0,35 & 3,07 & $-6,00$ & 6,00 & $-1,22$ & 1,93 \\
\hline $\mathbf{1 2}<$ Monat $\leq \mathbf{2 4}$ & $20 \mathrm{G}$ & 44 & $-1,16$ & 3,14 & $-11,00$ & 7,00 & $-2,12$ & $-0,21$ \\
\hline $\mathbf{1 2}<$ Monat $\leq \mathbf{2 4}$ & $23 \mathrm{G}$ & 6 & $-1,16$ & 1,21 & $-2,50$ & 1,00 & $-2,44$ & 0,10 \\
\hline
\end{tabular}


Auch wenn die statistische Auswertung zeigte, dass der Druck bei 20-G-Vitrektomien auch bei nicht-operierten Augen im Mittel minimal sank, konnte über den t-Test (Abbildung 25) kein signifikanter Unterschied zwischen den Operationstechniken (23 G/20 G) festgestellt werden. Jedoch muss gleichzeitig darauf hingewiesen werden, dass für diese Analyse weniger Augen als bei der Gruppe der operierten Augen zur Verfügung standen, da postinterventionell nicht immer auch das Partnerauge mitgemessen wurde.

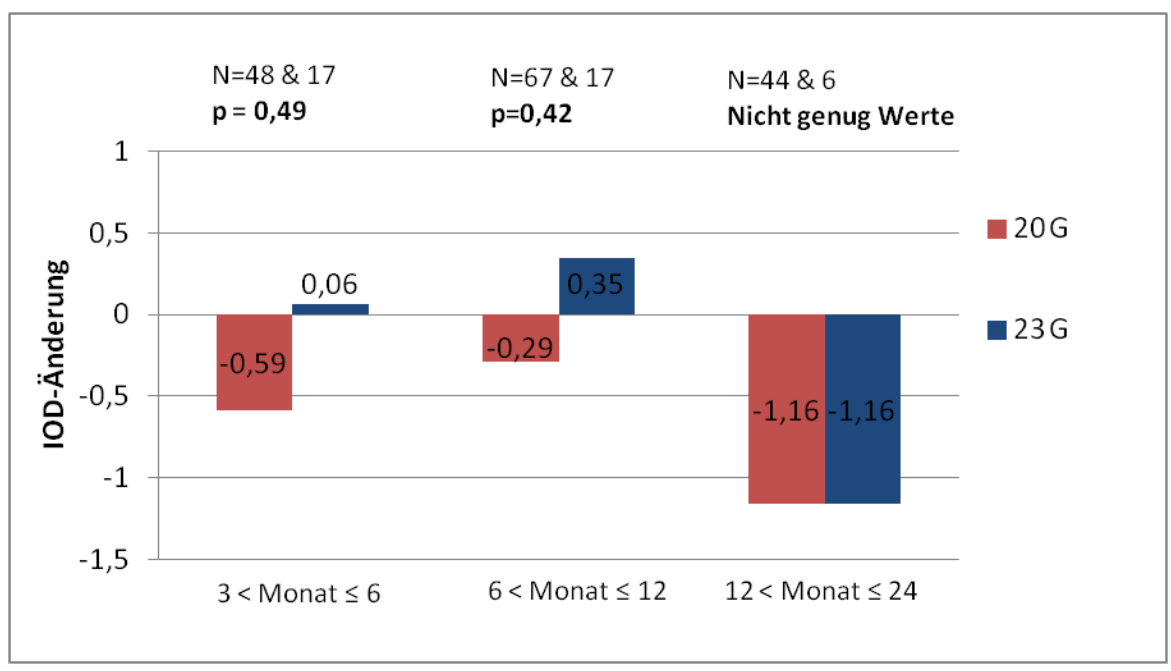

Abbildung 25: Unverbundene t-Tests für die Vitrektomie des nicht-operierten Auges

\subsubsection{Differenz der IOD-Änderung unter dem Einfluss der Vitrektomgröße $(20 G / 23 G)$}

Da bei den operierten Augen der IOD nach 20-G-Vitrektomie signifikant gesunken war, wurde nun analysiert, ob diese Änderung auch im Vergleich mit dem nicht-operierten Auge bestehen blieb, d. h. ob das operierte Auge einen stärkeren Druckabfall als das nicht-operierte aufwies. Hierfür wurde jeweils für $20 \mathrm{G}$ und $23 \mathrm{G}$ die Differenz der IOD-Änderung zwischen operiertem und nicht-operiertem Auge berechnet:

Differenz der IOD-Änderung = IOD- ̈̈nderung ${ }_{\text {nicht-operiert }}-$ IOD-Änderung ${ }_{\text {operiert }}$

Um die statistische Signifikanz der Differenz zu analysieren, wurde zusätzlich ein verbundener t-Test durchgeführt. Für den Zeitraum von 6-12 Monaten ergab sich auch hier ein statistisch signifikanter Unterschied zwischen den Augen ( $\mathrm{p}$-Wert $=0,05$, Tabelle 23) für einen durchschnittlichen Mittelwert der Differenz von 0,59 mmHg. Dies bestätigte die vor- 
liegenden Ergebnisse der Subgruppenanayse bezüglich der Vitrektomgröße: Zum Zeitpunkt 6-12 Monate sank der IOD signifikant nach 20-G-ppV, nicht jedoch nach 23-G-ppV.

Tabelle 23: Statistische Auswertung der Differenz der IOD-Änderung

\begin{tabular}{|c|c|c|c|c|c|c|}
\hline Variable & $\mathrm{N}$ & Mean & SEM & Min & Max & p-Wert \\
\hline $\begin{array}{c}\mathbf{2 0} \text { G } \\
\mathbf{3}<\text { Monat } \leq \mathbf{6}\end{array}$ & 47 & 0,5 & 0,43 & -6 & 9 & 0,77 \\
\hline $\begin{array}{c}\mathbf{2 0} \text { G } \\
\mathbf{6}<\text { Monat } \leq \mathbf{1 2}\end{array}$ & 67 & 0,59 & 0,31 & -7 & 8,5 & 0,05 \\
\hline $\begin{array}{c}\mathbf{2 3 ~ G} \\
\mathbf{3}<\text { Monat } \leq \mathbf{6}\end{array}$ & 17 & $-0,03$ & 0,76 & -7 & 6 & 0,97 \\
\hline $\begin{array}{c}\mathbf{2 3} \text { G } \\
\mathbf{6}<\text { Monat } \leq \mathbf{1 2}\end{array}$ & 17 & 0,12 & 0,74 & -6 & 5 & 0,87 \\
\hline
\end{tabular}

\subsubsection{Einfluss der Endotamponade}

Auch wenn über die ANCOVA-Analyse kein signifikanter Einfluss der Endotamponade auf die IOD-Änderung festgestellt wurde, wurden die Endotamponaden-Subgruppen nochmals in den folgenden Abbildungen graphisch aufgetragen.

In Abbildung 26, Abbildung 27 und Abbildung 28 wurden die IOD-Änderungen für die verschiedenen Zeitintervalle mit Standardabweichung dargestellt. Bei Vitrektomien ohne Endotamponade zeigte sich zu allen Zeitpunkten eine Tendenz zu sinkenden IOD-Werten.

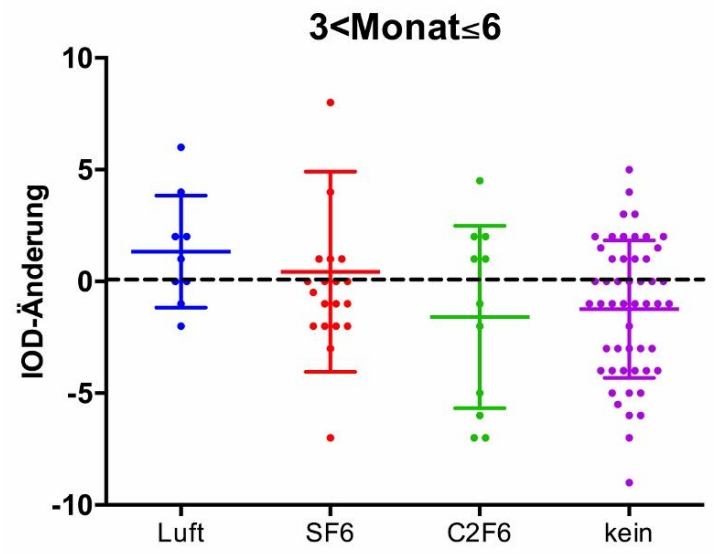

\begin{tabular}{|c|c|c|c|}
\hline Endotamponade & $\mathrm{N}$ & Mean & SD \\
\hline Luft & 9 & 1,33 & 2,50 \\
\hline SF6 & 20 & 0,43 & 4,48 \\
\hline C2F6 & 11 & $-1,59$ & 4,08 \\
\hline Keine & 51 & $-1,25$ & 3,07 \\
\hline
\end{tabular}

Abbildung 26: IOD-Änderung Endotamponaden: Zeitraum 3-6 Monate 


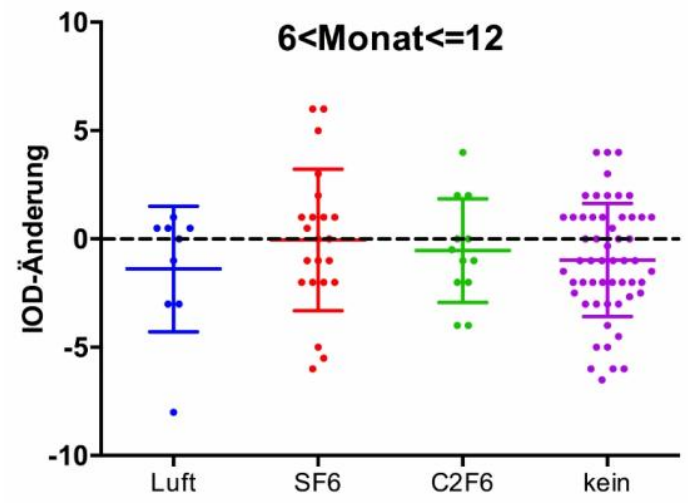

\begin{tabular}{|c|c|c|c|}
\hline Endotamponade & $\mathrm{N}$ & Mean & SD \\
\hline Luft & 9 & $-1,39$ & 2,90 \\
\hline SF6 & 22 & $-0,05$ & 3,27 \\
\hline C2F6 & 12 & $-0,54$ & 2,39 \\
\hline Keine & 55 & $-0,97$ & 2,61 \\
\hline
\end{tabular}

Abbildung 27: IOD-Änderung Endotamponaden: Zeitraum 6-12 Monate

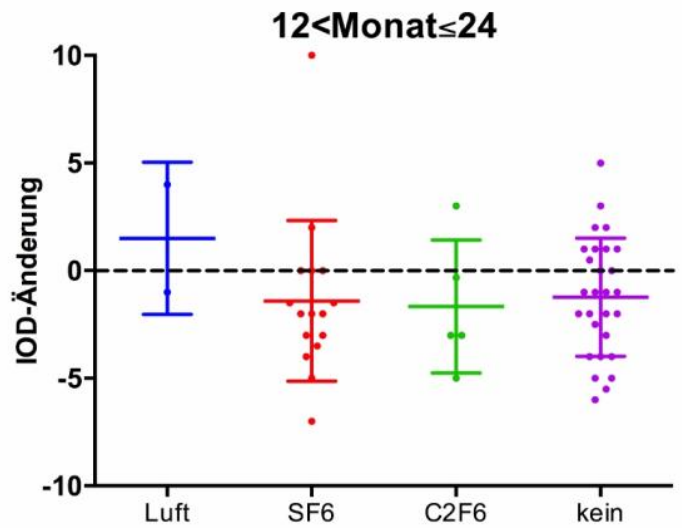

\begin{tabular}{|c|c|c|c|}
\hline Endotamponade & $\mathrm{N}$ & Mean & SD \\
\hline Luft & 2 & 1,5 & 3,54 \\
\hline SF6 & 16 & $-1,41$ & 3,73 \\
\hline C2F6 & 5 & $-1,67$ & 3,09 \\
\hline Keine & 28 & $-1,23$ & 2,74 \\
\hline
\end{tabular}

Abbildung 28: IOD-Änderung Endotamponaden: Zeitraum 12-24 Monate

In Tabelle 24 sah man jedoch in der Übersicht aller Patienten, die sich einer 20-G- oder 23-GVitrektomie unterzogen, dass die meisten Patienten (95\%), die keine Endotamponade erhielten, auch mit der 20-G-Technik operiert wurden. Dies vermischt sich mit dem bereits festgestellten Ergebnis, dass der Druck nach 20-G-Operationen tendenziell sinkt. Das Patientenkollektiv, das keine Endotamponade erhalten hat, wurde durch den überproportional höheren Anteil an 20-G-Vitrektomien verzerrt. Damit reduziert sich die Aussagekraft dieser Subgruppenanalyse.

Tabelle 24: Übersicht Vitrektomgröße und jeweilige Endotamponade

\begin{tabular}{|c|c|c|c|c|}
\hline Vitrektomie & Luft & SF6 & C2F6 & Keine \\
\hline $20 \mathrm{G}$ & $10(53 \%)$ & $62(85 \%)$ & $18(46 \%)$ & $112(95 \%)$ \\
\hline $23 \mathrm{G}$ & $9(47 \%)$ & $11(15 \%)$ & $21(54 \%)$ & $6(5 \%)$ \\
\hline
\end{tabular}




\section{Diskussion}

In bereits vielen Studien wurde der IOD-verändernde Einfluss einer Pars-plana-Vitrektomie untersucht. Unmittelbar postoperativ sind IOD-Spitzen kein unbekanntes Phänomen. Die Inzidenz des unmittelbaren IOD-Peaks variiert in der Literatur zwischen 8,4 und 92 Prozent (Weinberg et al. 1976; Thompson et al. 1996; Han et al. 1989; Chen und Thompson 1997; Desai et al. 1997; Chen 1998; Anderson et al. 2006; Framme et al. 2012; Hasegawa et al. 2012). Die Autoren erklärten sich dies durch vermehrte Fibrinbildung, mechanische Verlegung des TMW (fragmentierte Erythrozyten, Entzündungszellen, Blutung, Linsenfragmente), Silikonöltamponade, Gasexpansion, TGF-ß-Behandlung, die Operation selbst, Kopftief-Position sowie angewandte Operationstechniken mit Skleraeindellung, Fundus-Photokoagulation und Kryotherapie. Auch längerfristig wurde bereits mehrfach der VitrektomieEinfluss, jedoch auf einen IOD-Anstieg in Hinblick auf die Entstehung sekundärer Offenwinkelglaukome untersucht. Hier findet man kontroverse Ergebnisse, ob postoperativ das Risiko eines IOD-Anstiegs und damit eines Sekundärglaukoms steigt. Manche Studien fanden einen signifikanten längerfristigen Anstieg des IOD nach ppV mit Inzidenzangaben von 4,2 bis 20 Prozent (Chang 2006; Koreen et al. 2012; Luk et al. 2009; Wu et al. 2014; Toyokawa et al. 2015; Fujikawa et al. 2014; Lalezary et al. 2014), andere konnten diesen IOD-Anstieg nicht nachweisen (Yu et al. 2010; Ki-I et al. 2013; Lalezary et al. 2011) bzw. konnten sogar z. T. einen IOD-Abfall feststellen, was die Autoren selbst nicht erwarteten und sich auch nicht erklären konnten (Lalezary et al. 2011). Als Ursache für den möglichen IOD-Anstieg wurde oxidativer Stress auf das TMW, Zustand nach Kataraktextraktion und dadurch bedingte Pseudo- oder Aphakie sowie eine positive Familienanamnese gesehen (Siegfried et al. 2010). Da bisher immer der IOD-Anstieg als postoperative Komplikation untersucht wurde, klinische Beobachtungen aber andere waren, gab dies Anlass für die vorliegende Studie, den mittel- bis längerfristigen IOD-Abfall zu untersuchen.

Für vorliegende Studie wurden 249 vitrektomierte Patienten auf den mittel- bis längerfristigen Einfluss der Vitrektomie bei MF und EG in Hinsicht auf eine Drucksenkung untersucht. Hierfür wurden stringente Ausschlusskriterien festgelegt, welche darauf zielten, andere Einflussgrößen auf den IOD möglichst zu eliminieren. Ausgeschlossen wurden Patienten mit zu kurzer Nachbeobachtungszeit von unter 2 Monaten (mögliche Nachwirkung unmittelbar postoperativer IOD-Anstiege), einem Alter unter 18, einer bekannten Steroide-Response (möglicher IOD-Anstieg durch postoperative Steroid-Nachbehandlung), Z. n. Uveitis oder schwerem Trauma (erhöhtes Risiko für IOD-Anstieg), bekanntem Glaukom (nicht-Vitrektomie- 
bedingter IOD-Anstieg), intra- oder postoperativer Katarakt-Operation (IOD-senkende Wirkung der Katarakt-Operation), vorangegangener Vitrektomie, ausgiebiger Netzhaut-Kryokoagulation (IOD-senkender Effekt der Kryokoagulation), kombinierter Cerclage oder Plombe (denkbarer Einfluss auf Kammerwinkel), intraoperativer panretinaler Laserkoagulation (mögliche IOD-Senkung durch chorioretinale Narben), Silikonöltamponade (Risiko IOD-Anstieg durch Öl), Steroidinjektion (möglicher IOD-Anstieg) oder Thrombozytenkonzentrat intraoperativ, Ablatio retinae (IOD-Senkung durch Ablatio), Glaskörperblutung/ Makulablutung (IOD-Anstieg durch Erythrozytenfreisetzung), diabetischer Retinopathie (Risiko IOD-Anstieg bei schwerer ischämischer Retinopathie).

Zu definiertem primärem Endpunkt nach 6-12 Monaten waren unter den 249 vitrektomierten Patienten noch 98 operierte und 84 nicht-operierte Augen. In der Gruppe der operierten Augen lag der durchschnittliche IOD bei 14,91 $\pm 2,80 \mathrm{mmHg}$ und bei 15,25 $\pm 2,49 \mathrm{mmHg}$ in der Gruppe der nicht-operierten Kontrollaugen. Die errechnete Differenz zwischen der IODÄnderung des nicht-operierten Auges zu der des operierten Auges ergab einen Wert von $0,49 \mathrm{mmHg}$, erbrachte jedoch keine statistische Signifikanz $(\mathrm{p}$-Wert $=0,0893)$ Von den möglichen Einflussfaktoren auf den IOD wurden untersucht: Linsenstatus, Vitrektomgröße, Endotamponade, Alter, Geschlecht, sphärisches Äquivalent, Anzahl der drucksenkenden Augentropfen und die Operation selbst. Hier konnte ein signifikanter Unterschied zwischen der 20-G- und 23-G-Vitrektomgröße im Zeitraum von 6-12 Monaten für das operierte Auge festgestellt werden $(\mathrm{p}$-Wert $=0,04)$. 20-G-vitrektomierte Augen zeigten einen signifikanten Druckabfall, 23-G-operierte Augen blieben auf dem präoperativen Niveau stabil. Zu den definierten sekundären Endpunkten konnte kein statistisch signifikanter Unterschied zwischen $20 \mathrm{G}$ und $23 \mathrm{G}$ nachgewiesen werden.

\subsection{Diskussion von Material und Methoden}

\subsubsection{Studienaufbau}

Um die Störfaktoren möglichst gering zu halten, wurden die Ein- und Ausschlusskriterien in vorliegender Studie vor der Datenerhebung genau definiert. In die Studie wurden 2 Krankheitsbilder, nämlich MF und EG einbezogen. Dies stellt einen Vorteil unserer Studie dar, da viele der sonst vorliegenden Studien Patientenkollektive mit breiterem Krankheitsspektrum eingeschlossen hatten. Als Ausschlusskriterien wurden Krankheitsbilder (Glaukom, Uveitis, diabetische Retinopathie, Makulaödem, Ablatio Retinae, Glaskörper-/Makulablutung und 
Steroide-Responder), Operationstechniken (Laser, Kryokoagulation, Silikonöl, Cerclage, Plombe, Thrombozytenkonzentrate und Steroidinfiltration) sowie kombinierte Operationen (Kataraktextraktion, Re-ppV) gewählt, welche bekanntermaßen den IOD beeinflussen (meist steigern) und dadurch die Studie in Hinblick auf die IOD-Senkung hätten beeinflussen können. Hierdurch konnten mögliche nicht-vitrektomie-bedingte Einflussfaktoren reduziert werden. Jedoch konnte nicht sicher davon ausgegangen werden, dass die Anamnese in den Akten komplett erhoben wurde. Mögliche Fehlerquellen lagen daher in der ärztlichen Dokumentation der genutzten Akten. Andere Studien verwendeten selten Patientenaugen, die alleinig vitrektomiert wurden - häufig in Kombination mit Kataraktextraktion, Kryokoagulation, Laser und Skleraeindellung. Dies stellte wiederum einen Vorteil unserer Studie dar, da gerade Pseudophakie als Risikofaktor für die langfristige Entwicklung eines Offenwinkelglaukoms nach Vitrektomie diskutiert wird. In unserer Studie wiesen $92,8 \%$ der Patientenaugen einen phaken Linsenstatus auf; der protektive Effekt der Linse könnte vor oxidativem Stress am TMW und damit vor einem IOD-Anstieg schützen.

Definierte Untersuchungsparameter wie der Linsenstatus, intraoperative Endotamponaden, Peelingfarbstoffe, Applikation von IOD-senkenden AT, Vitrektomgröße und sphärisches Äquivalent wurden als mögliche Einflussfaktoren auf den IOD erfasst und analysiert. Auch hier legten wir ein Augenmerk auf Untersuchungsparameter, die möglicherweise den IOD beeinflussen können. Gerade Endotamponaden (Hasegawa et al. 2012; Thompson et al. 1996; Fujikawa et al. 2014), Pseudophakie (Chang 2006; Koreen et al. 2012; Luk et al. 2009), Peelingfarbstoffe, z. B. ICG, (Mi und Thompson 2015) und Vitrektomgröße wurden in der aktuellen Literatur als IOD-beeinflussend diskutiert. Auch das sphärische Äquivalent wurde bereits untersucht, jedoch ohne einen statistisch signifikanten Einfluss auf den IOD feststellen zu können (Luk et al. 2009). Durch die Wahl ähnlicher Parameter wie in der bereits vorhandenen Literatur war es möglich, unsere Ergebnisse besser in den Stand der Forschung einzuordnen und zu vergleichen.

Festgelegte Endpunkte waren die IOD-Änderungen von präoperativ zu definierten Zeiträumen. Das Intervall des primären Endpunktes wurde auf 6-12 Monate postinterventionell definiert. Ausschlaggebend für die Festlegung auf diesen Zeitraum war, dass die Nachwirkungen der postoperativen Phase bereits abgeklungen und der IOD-Wert zu gewähltem Zeitpunkt konsolidiert sein müsste. Zudem sollte das Zeitintervall lang genug sein, um erste Komplikationen bemerken zu können. Ein längeres Zeitintervall wäre jedoch nicht sinnvoll gewesen, da das Patientenkollektiv sonst zu klein geworden wäre. Die sekundären Endpunkte 
wurden zwischen 3 und 6 Monaten sowie 12 und 24 Monaten festgelegt. Hier zeigte sich in der 12-24-Monate-Gruppe bereits das Problem einer stark abnehmenden Gruppengröße, welche die statistische Auswertung erheblich behinderte.

\subsubsection{Limitierende Faktoren des Studiendesigns}

Die vorliegende Studie ist eine retrospektive Kohortenstudie, in der das Partnerauge als Kontrollgruppe dient. Ein Nachteil einer retrospektiven Studie kann in der mangelnden Datenqualität bestehen, da die untersuchten Merkmale nicht immer und wenn ggf. auch fehler- oder nur lückenhaft dokumentiert sind. Eine prospektive Studie könnte diese Einflussfaktoren besser kontrollieren; anhand vorliegender Ergebnisse kann nur eine Hypothese aufgestellt werden. Daher müsste eine prospektive Studie zur Prüfung der Hypothese durchgeführt werden. Zur teilweisen Kompensation der schlechten Kontrolle möglicher Einflussfaktoren in einer retrospektiven Studie wurden für diese Arbeit stringente Einschlusskriterien festgelegt, um die Studie so sauber und klar wie möglich zu halten. Zudem wurde ein großes Patientenkollektiv in die Studie eingeschlossen.

Ein weiterer limitierender Faktor vorliegender Studie ist, dass nur die in den Akten dokumentierten Messergebnisse, die auch in der UMG erhoben worden waren, verwendet werden konnten. Mögliche Nachkontrollen erfolgten häufig nicht ausreichend lange genug postoperativ oder ambulant beim niedergelassenen Ophthalmologen, sodass ein langfristiger IODWert nicht mehr in der Akte dokumentiert war und somit auch nicht in der Studie verwendet werden konnte. Für den Zeitraum von 12-24 Monaten kam es daher aufgrund der langen Nachbeobachtungszeit zu einem großen Datenschwund.

Auch die festgesetzten Zeitintervalle der Kontrollmessung fanden nicht zu standardisierten Zeitpunkten statt, sondern wurden durch die festgelegten Endpunkte in größere Zeitintervalle zusammengefasst. Dies macht eine genaue Bestimmung des Zeitpunktes der IOD-Senkung schwierig, wieder kann nur eine Hypothese aufgestellt werden.

Des Weiteren wurde das Partnerauge nicht immer mitgemessen, sodass dieses nur eingeschränkt als Kontrolle genutzt werden konnte. Lediglich wenn der IOD im Partnerauge mitbestimmt wurde, konnte dieser Wert mit in die Studie aufgenommen werden.

In prospektiven Studien wird in der Regel zu einem Zeitpunkt der IOD mehrfach gemessen und gemittelt, um Messschwankungen klein zu halten. Dies ist bei einer retrospektiven Studie nicht möglich. Es muss daher davon ausgegangen werden, dass wir die Messschwankungen 
der IOD-Messung (ca. $\pm 1-2 \mathrm{mmHg}$ ) in unseren Daten zum Tragen kommen. Auch aus diesem Grund wurde versucht, eine möglichst große Gruppengröße zu realisieren, um den Einfluss dieses Messrauschens auf die Detektion von IOD-Änderungen zu minimieren.

\subsection{Diskussion der Ergebnisse}

\subsubsection{Ergebnisse im Vergleich zum aktuellen Stand der Forschung}

\subsubsection{Kurzfristiger IOD-Anstieg}

Ein IOD-Peak unmittelbar postoperativ ist kein unbekanntes Phänomen und bereits 1976 durch Weinberg und Kollegen beschrieben worden. Postoperative Entzündung, mechanische Verlegung des TMW durch fragmentierte Erythrozyten und frische Blutungen, sowie verbleibende Linsenfragmente wurden als Ursache beschrieben (Weinberg et al. 1976). Auch TGF-ß (Thompson et al. 1996; Desai et al. 1997), Operationstechniken wie Skleraeindellung, Fundus-Photokoagulation und Linsenextraktion (Han et al. 1989; Framme et al. 2012), steigendes Patientenalter, dehnbare Gaskonzentrationen, Einsatz von C3F8 (Chen und Thompson 1997; Chen 1998) und Silikonöltamponaden (Framme et al. 2012) wurden als Ursachen untersucht und festgestellt. Ebenfalls konnte ein Unterschied des IOD-Anstiegs abhängig von zu Grunde liegender Krankheit festgestellt werden. So zeigten Augen mit MF eine niedrigere Inzidenz eines IOD-Anstiegs als solche mit Retinaablösung (Hasegawa et al. 2012). Eine Studie von Anderson und Kollegen konnte hingegen keinen Einfluss der Endotamponade, Silikonöl, Skleraeindellung und Endolaser auf den IOD eruieren und sah das Risiko eines IOD-Peaks als niedrig an (Anderson et al. 2006). Eine präzise Ursache des IODAnstieges postoperativ konnte bei sehr unterschiedlichem Studiendesign mit unterschiedlichen Ein- und Ausschlusskriterien bisher nicht gefunden werden. In dieser retrospektiven Studie wurde ebenfalls der IOD-Wert postoperativ erhoben. Dieser wurde aus den Mittelwerten aller Messungen gebildet und konnte Werte von unmittelbar bis 6 Tage postoperativ beinhalten. Der durchschnittliche IOD-Wert lag postoperativ bei 17,6 $\pm 5,42 \mathrm{mmHg}$. Im Vergleich zeigten die präoperativen Werte, welche bis 6 Tage vor Operation erhoben wurden, einen Mittelwert von 15,67 $\pm 2,81 \mathrm{mmHg}$. Hieraus ließ sich ein Druckunterschied von 1,93 mmHg errechnen. Ein verbundener t-Test der präoperativen zu den postoperativen IODWerten konnte eine statistisch signifikante Druckerhöhung nachweisen (p-Wert $<0,001$ ). Damit konnten wir in unserer Studie einen moderaten IOD-Peak unmittelbar postoperativ 
bestätigen, jedoch lag unser Augenmerk auf dem mittel- bis längerfristigen Einfluss der ppV auf den IOD, sodass wir dieses Ergebnis nicht weiter analysierten.

\subsubsection{Langfristiger IOD-Verlauf}

In der vorhandenen Literatur gibt es bisher einige Studien, die die Inzidenz der Entstehung eines Offenwinkelglaukoms als Langzeitkomplikation untersuchten. Die post-ppV-Inzidenz des Offenwinkelglaukoms wird in der Literatur mit 4,2-20\% angegeben. Chang und Kollegen hatten als Erste den Verdacht, dass sich sekundäre Offenwinkelglaukome als Langzeitfolge der ppV entwickeln könnten und zeigten, dass die Pseudophakie einen Risikofaktor darstellt (Chang 2006). Im physiologischen Zustand herrscht ein Sauerstoffgradient (Retina > Linsenkern) im Auge, der durch den Glaskörper und die Linse metabolisiert wird. Sind diese physiologischen Schutzmechanismen durch stattgehabte Operation nicht mehr vorhanden und der Sauerstoffgehalt post-ppV ist auf ein Zehnfaches erhöht (Holekamp et al. 2005), wird vermutet, dass oxidativer Stress die Zellen des TMW und den vorderen Kammerwinkel modulieren und damit den Kammerwasserabfluss behindern (Siegfried et al. 2010). Diese Hypothese konnte auch durch andere Studien unterstützt werden (Koreen et al. 2012; Luk et al. 2009; Wu et al. 2014; Toyokawa et al. 2015; Fujikawa et al. 2014; Lalezary et al. 2014). Auch eine positive Familienanamnese (Wu et al. 2014), die Kopftief-Position nach ppV bei MF, intraoperative Gastamponaden (Fujikawa et al. 2014), Retinaablösung als Grunderkrankung (Yamamoto et al. 2016) und Triamcoloninjektionen (Yamashita et al. 2007) wurden als Risikofaktor für die Entstehung eines Offenwinkelglaukoms beschrieben.

In anderen Studien konnten jedoch weder eine erhöhte Inzidenz für sekundäre Glaukome noch ein Linsenstatus-abhängiger IOD-Unterschied nach $\mathrm{ppV}$ festgestellt werden (Mi und Thompson 2015; Yu et al. 2010; Lalezary et al. 2011). Yamamoto und Kollegen konnten keinen Einfluss der Gasendotamponade auf den IOD feststellen (Yamamoto et al. 2016). In anderen Studien konnte ein signifikanter IOD-Abfall im Vergleich zum Kontrollauge nach 3 Monaten (Ki-I et al. 2013) und nach 3 bis 6 Monaten (Mi und Thompson 2015) festgestellt werden. Lalezary und Kollegen konnten 2011 in ihrer Studie bei 35 der 101 Studienaugen einen IOD-Abfall feststellen (34,7\%), obwohl sie eigentlich einen Anstieg erwartet hätten. Diese vermuteten als Ursache für das beobachtete Phänomen eine verminderte Kammerwasserproduktion und vermehrten Kammerwasserabfluss (Lalezary et al. 2011). Weitere Vermutungen waren eine vermehrte arterielle Konstriktion durch den erhöhten oxidativen Stress, 
was den Blutfluss zum Ziliarkörper und damit die Kammerwasserproduktion vermindert. Auch ein Einfluss auf den episkleralen Venendruck wurde vermutet. Jede der vorliegenden Studien hatte andere Ein- und Ausschlusskriterien. Da bisher aber immer nur zufällig ein IOD-Absinken festgestellt wurde, konnte keine vorliegende Studie zuverlässig den IODAbfall als Langzeitfolge der ppV untersuchen.

Die Analyse des primären Endpunkts unserer Studie, das heißt die IOD-Änderung zwischen 6-12 Monaten zum präoperativen Wert, ergab einen leichten Abfall des Druckes: -0,66 $\mathrm{mmHg}$ für das operierte Auge und $-0,17 \mathrm{mmHg}$ für das nicht-operierte Auge, was unter Bildung der Differenz einen Unterschied von $0,5 \mathrm{mmHg}$ ausmachte. Es zeigte sich kein statistisch signifikanter Unterschied zwischen der Änderung der operierten und der nichtoperierten Augen $(\mathrm{p}=0,0893)$. Auch die Analyse der sekundären Endpunkte, der IODÄnderung nach 3-6 Monaten und 12-24 Monaten, ergab ebenfalls keine statistisch signifikante IOD-Änderung gegenüber der Änderung am nicht-operierten Auge. Für die Subgruppe der 20-G-Vitrektomien zeigte sich allerdings eine signifikante IOD-Senkung der operierten gegenüber den nicht-operierten Augen nach 6-12 Monaten ( $p=0,05$; siehe Kap. 4.2.2).

Wie bereits beschrieben fanden auch Lalezary und Kollegen in ihrer retrospektiven Kohortenstudie (2011) unerwartet bei 34,7 \% der Studienaugen einen IOD-Abfall. Die Studie schloss jedoch ein breites Krankheitsspektrum ein und die Ausschlusskriterien waren nicht sehr stringent gewählt. So waren zum Beispiel viele der eingeschlossenen Patienten Diabetiker und Katarakt-Operationen nach Vitrektomie waren nicht ausgeschlossen, was einen zusätzlichen Einfluss auf IOD-Änderungen haben könnte (Lalezary et al. 2011).

Neben Lalezary et al. (2011) wurde ein Absinken des IOD auch von Mi und Thompson (2015) beschrieben. Die Autoren konnten in ihrer retrospektiven Fallstudie nach 3-6 Monaten einen signifikanten IOD-Abfall feststellen, welcher nach 1 und 2 Jahren postoperativ im operierten Auge weiterhin niedriger war als im nicht-operierten, jedoch nicht mehr statistisch signifikant. Indikation zur Operation waren ebenfalls an MF $(\mathrm{N}=100)$ und EG $(\mathrm{N}=134)$ erkrankte Augen und auch hier waren 73,1\% der Studienaugen phak. Damit ähnelte diese Studie unserer hier vorgestellten Untersuchung. Ähnlich wie in unserer Studie beobachteten Mi und Thompson einen leichten IOD-Abfall am Partnerauge, welchen sie sich nicht erklären konnten.

Ki-I und Kollegen (2013) fanden an MF ( $=44)$ und EG $(\mathrm{N}=41)$ nach kombinierter Phakoemulsifikation und Linsenimplantation nach 3 Monaten einen statistisch signifikanten IOD- 
Abfall im Vergleich zum Partnerauge, jedoch zu keinem weiteren Zeitpunkt in ihrem Nachbeobachtungszeitraum von 5 Jahren (Ki-I et al. 2013). Auch wir konnten eine Tendenz zum Druckabfall im Vergleich zum Partnerauge nach 3 Monaten feststellen, jedoch ohne statistische Signifikanz. Ein Vergleich der Ergebnisse war jedoch schwierig, da hier kombinierte Katarakt-Operationen mit Vitrektomien durchgeführt wurden.

Zusammenfassend betrachtet fanden sich in den verfügbaren Studien Hinweise, welche die von uns beobachtete Tendenz zu sinkenden IOD-Werten nach Vitrektomie bestätigten.

\subsubsection{Diskussion der Einflussfaktoren der Subgruppenanalyse}

Mögliche Einflussfaktoren wie Linsenstatus, Vitrektomgröße, Endotamponade, Geschlecht, Alter, sphärisches Äquivalent, die Anzahl der drucksenkenden Augentropfen sowie die Operation selbst wurden über ANCOVA-Analyse auf einen möglichen Einfluss auf den IOD untersucht. Hierfür wurde die IOD-Änderung zum Zeitpunkt des primären Endpunktes (6-12 Monate) verwendet.

In Bezug auf den Linsenstatus gibt es in der Literatur bereits einige Studien, die nach Kataraktextraktion und dadurch bedingter Pseudo- oder Aphakie einen IOD-Anstieg und das Risiko eines Offenwinkelglaukoms nach Vitrektomie aufzeigten. Dies wurde durch den fehlenden protektiven Effekt vor oxidativem Stress erklärt (Chang 2006; Koreen et al. 2012; Luk et al. 2009; Toyokawa et al. 2015; Wu et al. 2014; Lalezary et al. 2014; Fujikawa et al. 2014). Andere Studien konnten keinen Einfluss des Linsenstatus auf den IOD finden (Lalezary et al. 2011; Mi und Thompson 2015; Yu et al. 2010). In unserer Studie waren 92,8 \% phak, ein statistisch signifikanter Einfluss des Linsenstatus auf die IOD-Änderung konnte nicht festgestellt werden. Allerdings erlaubte die starke Ungleichverteilung zwischen den beiden Gruppen phak (natürliche Linse) und pseudophak (Kunstlinse nach Katarakt-Operation) keine valide Aussage. Auch Mi und Thompson untersuchten vor allem phake Augen in ihrer Studie $(73,1 \%)$. Sie konnten kein statistisch signifikantes Risiko für die Entwicklung eines Offenwinkelglaukoms nachweisen. Changs Theorie des protektiven Effektes der Linse vor oxidativem Stress zweifelten die Autoren an, da Chang in seiner Studie eine große Anzahl Diabetiker aufgenommen hatte und Holekamp und Kollegen in einer weiteren Studie feststellten, dass diabetische Augen im Gegensatz zu nicht-diabetischen Augen ein hypoxischeres Niveau im Glaskörper aufweisen (Holekamp et al. 2006). Diabetische Augen müssten daher 
in Bezug auf die Entstehung eines Offenwinkelglaukoms protektiv sein, da der oxidative Stress reduziert ist. In Changs Studie war Diabetes daher ein möglicher Confounder.

Der Einflussfaktor Vitrektomgröße, das heißt ob mit 20-G- oder 23-G-Instrumenten operiert wurde, wurde ebenfalls bereits in der Literatur untersucht und beschrieben. Eine Hypotonieneigung nach 23-G-ppV wurde in der Literatur am ersten Tag postoperativ mit einer Inzidenz von 2,8-10\% angegeben (Fine et al. 2007; Gupta et al. 2008; Lott et al. 2008; Ahn et al. 2012; Misra et al. 2009; Zhang et al. 2015). Bei 20-G-vitrektomierten Augen konnte keine langanhaltende Hypotonie (> 6 Stunden) (Framme et al. 2012), sondern eine Hypertonieneigung von 21,6\% am ersten postoperativen Tag (Ahn et al. 2012) nachgewiesen werden. Ursächlich für den postoperativen Unterschied wurde die nahtlose 23-G-Wunde gesehen. Diese dient als Ventil des Druckgradienten und beugt somit über kleinste Leckagen einer Hypertonie vor. Des Weiteren wurde eine vermehrte Entzündungsreaktion nach 20-GOperation und damit Steigerung des IOD in Erwägung gezogen. Krishnan und Kollegen konnten 2013 in einer vergleichenden Studie an phakovitrektomierten Patienten keinen IODUnterschied zwischen $20 \mathrm{G}$ und $23 \mathrm{G}$ nach zwei bis drei Monaten feststellen (Krishnan et al. 2013). Zhang und Kollegen sahen bis auf ein postoperatives Hypotonierisiko nach 23-G-ppV keinen signifikanten Unterschied zwischen 20-G- und 23-G-Vitrektomierten bis 6 Monate postoperativ (Zhang et al. 2015). In vorliegender Studie zeigte sich, dass die Vitrektomgröße $20 \mathrm{G}$ einen signifikanten Einfluss nach 6-12 Monaten auf die IOD-Änderung nahm, nicht hingegen die 23-G-Operation. Die IOD-Änderung zwischen 20-G- (-1,02 mmHg) und 23-G$\mathrm{ppV}(0,44 \mathrm{mmHg})$ ergab für den Zeitraum 6-12 Monate einen statistisch signifikanten Unterschied zwischen den zwei Gruppen (p-Wert = 0,041). Die IOD-Werte nach 23-G-ppV waren nahezu stabil zu prä-OP-Werten.

Das Ergebnis unserer Untersuchung, dass 20-G-vitrektomierte Augen in der Langzeit-Nachbeobachtung eine signifikante IOD-Senkung aufwiesen, bei 23-G-Vitrektomie aber eher stabil blieben, ist sehr interessant, müsste aber in einer kontrollierten prospektiven Studie überprüft werden. Vor allem die Tatsache, dass in unserer Studie das 23-G-Patientenkollektiv deutlich kleiner war als das 20-G-Kollektiv, könnte die Aussagekraft unserer Studie limitieren.

Auch die Endotamponade wurde bereits als Einflussfaktor auf den IOD untersucht. Fujikawa und Kollegen vermuteten einen durch Gastamponaden getriggerten IOD-Anstieg, da diese zu Freisetzung erhöhter Konzentrationen inflammatorischer Zytokine führen, was wiederum zu mechanischer Verlegung des TMW führen kann (Fujikawa et al. 2014). Yamamoto und Kollegen fanden hingegen keinen Einfluss der Gastamponade auf den IOD (Yamamoto et al. 
2016). In unserer Studie ergab sich, dass Augen ohne Endotamponade in der Tendenz niedrigere Drücke aufwiesen als solche mit SF6, C2F6 und Luft. Sowohl zum Zeitpunkt des primären Endpunkts als auch zu denen der sekundären Endpunkte zeigte sich eine Tendenz zu sinkenden IOD-Werten. Jedoch unterzogen sich die meisten Patienten, die keine Endotamponade erhielten, einer 20-G-Operation (95\%), sodass hier eine mögliche Verzerrung vorliegen könnte.

Bezüglich des Geschlechts und des Alters vermutete Chen einen Einfluss auf den IOD-Peak unmittelbar postoperativ, konnte jedoch keinen statistisch signifikanten Einfluss nachweisen. Auch Hasegawa und Kollegen konnten keinen Einfluss feststellen (Chen 1998; Hasegawa et al. 2012). Eine weitere Studie von Chen und Thompson konnte einen signifikanten Einfluss des Alters auf den IOD unmittelbar postoperativ nachweisen (Chen und Thompson 1997). Das sphärische Äquivalent betreffend konnten Luk und Kollegen keinen Einfluss auf den Langzeit-IOD sehen (Luk et al. 2009). In unserer Studie konnte ebenfalls im Zeitraum von 6 bis 12 Monaten postoperativ kein signifikanter Einfluss auf die IOD-Änderung am operierten Auge festgestellt werden.

Die Krankheitsbilder Makulaforamen und epiretinale Gliose wurden nicht als Einflussfaktoren getrennt, da die meisten EG mit $20 \mathrm{G}$ operiert wurden $(85 \%)$ und somit die Einflussgröße des Vitrektoms eine Rolle gespielt haben könnte. Yamamoto und Kollegen untersuchten die Veränderungen des intraokularen Drucks auf lange Zeit (bis ca. 2 Jahre) für Retinaablösung, MF und EG. Hier zeigte sich ein deutlicher, langfristiger Druckanstieg für Retinaablösungen, der IOD blieb jedoch für MF und EG zu jeder Zeit ungefähr stabil (Yamamoto et al. 2016).

\subsubsection{Mögliche Ursachen eines IOD-Abfalls}

In vorliegender Studie konnte ein signifikanter IOD-Abfall im operierten Auge nach 6-12 $(\mathrm{p}=0,008)$ und 12-24 Monaten $(\mathrm{p}=0,007)$ nachgewiesen werden. Der IOD des nichtoperierten Auges zeigte ebenfalls eine Tendenz des Absinkens, dies war jedoch nur zum Zeitpunkt 12-24 Monate statistisch signifikant ( $p=0,008)$. Mögliche Erklärungen in der Literatur sind eine verminderte Kammerwasserproduktion, ein gesteigerter Kammerwasserabfluss und geänderte Messbedingungen durch veränderte Kornea-Oberflächenstrukturen (Lalezary et al. 2011). Aber auch die Veränderung der Sklerafestigkeit nach $p p V$ und ein darüber 
bedingter Einfluss auf den episkleralen Venendruck, welcher einen direkten Effekt auf den IOD hat, werden diskutiert.

Da in durchgeführter Subgruppenanalyse ein signifikanter Unterschied des IOD-Abfalls bei der 20-G- im Vergleich zur 23-G-Operation festgestellt wurde, stellten wir die Hypothese auf, dass die Operationstechnik den IOD beeinflusst. Mit dem 20-G-Vitrektom, welches starrer ist, kann man den Glaskörper besser bis in die Peripherie hinein abtragen. Dies gelingt weniger gut mit dem 23-G-Vitrektom, welches nicht ganz so rigide ist (Wimpissinger et al. 2008). Daher könnte eine mögliche Hypothese die Beeinflussung des uveoskleralen Abflusses sein, welcher durch Abtragung des Glaskörpers bis in die Peripherie mithilfe eines 20-G-Vitrektoms besser möglich ist. Hierzu findet man jedoch bisher keine Angaben in der Literatur. Deshalb müsste eine separate Studie die Frage nach dem Zusammenhang zwischen Vitrektomieverfahren und uveoskleralem Abfluss zu klären versuchen. Die Messung des uveoskleralen Abflusses ist allerdings technisch nur sehr schwierig zu realisieren. Zudem wäre diese Fragestellung zwar grundlagenwissenschaftlich sehr interessant, für die klinische Anwendung hätte sie aber keine Relevanz. Eine Drucksenkung von ca. 1,02 mmHg wäre kein Grund, eine Vitrektomie als drucksenkenden Eingriff durchzuführen. Auch wäre sie nicht groß genug, um zu empfehlen, bei Glaukompatienten, die eine Vitrektomie benötigen, lieber eine 20-G- als eine 23-G-Vitrektomie durchzuführen. Es überwiegen die Vorteile der 23-GVitrektomie, wie die fehlende Notwendigkeit, die Bindehaut zu eröffnen, was zum Beispiel weniger Nebenwirkungen für die Augenoberfläche hat.

Entgegen unseren Erwartungen sank auch der IOD des Kontrollauges, wenn auch weniger stark als der des vitrektomierten Auges. Dieses Phänomen konnten auch Mi und Thompson beobachten, konnten sich dies jedoch ebenso wenig wie wir erklären (Mi und Thompson 2015). 


\section{Zusammenfassung}

Ziel der Arbeit war es, den mittel- bis längerfristigen Einfluss der Vitrektomie bei MF und EG auf den IOD zu beurteilen. Hierbei hatten klinische Beobachtungen gezeigt, dass der Druck längerfristig sinkt, jedoch wurde bisher nur das Risiko der Entstehung eines Offenwinkelglaukoms untersucht. Des Weiteren sind die Angaben in der Literatur sehr kontrovers, was uns Anlass gab, vorliegende Studie durchzuführen.

In einer retrospektiven Fallserie wurden 249 Patientenaugen untersucht, das Partnerauge diente als Kontrollauge und Vergleichskohorte. Anhand von Patientenakten der Universitätsklinik Göttingen wurden die Daten im Archiv erhoben und im Vergleich zum Kontrollauge evaluiert. Ausgeschlossen wurden zahlreiche Faktoren, welche bekannterweise den IOD beeinflussen, um so mögliche Störfaktoren zu limitieren und den IOD-Verlauf postoperativ zuverlässiger beurteilen zu können. Mögliche Einflussfaktoren wurden zudem mittels ANCOVA-Analyse auf ihren Einfluss auf den IOD untersucht. Als primärer Endpunkt wurde die IOD-Änderung im Vergleich zum Partnerauge zum Zeitraum 6-12 Monate definiert. Sekundäre Endpunkte waren die IOD-Änderung im Vergleich zum Partnerauge nach 3-6 sowie 12-24 Monaten.

Unsere Ergebnisse zeigten zu allen festgelegten Zeitpunkten eine Tendenz zu sinkenden IODWerten im operierten Auge im Vergleich zum Kontrollauge. Nach 6-12 Monaten konnte die Tendenz des stärksten IOD-Abfalls festgestellt werden. Wenn man nur das operierte Auge betrachtete, konnte ein statistisch signifikanter IOD-Abfall für den Zeitraum 6-12 und 12-24 Monaten, im Vergleich zum präoperativen Wert über einen verbundenen t-Test errechnet werden. Interessanterweise kam es jedoch auch im Partnerauge jeweils zu einem IOD-Abfall, jedoch weniger ausgeprägt als im operierten Auge, wodurch sowohl der primäre, als auch die sekundären Endpunkte keinen signifikanten Druckabfall in Relation zum Partnerauge aufwiesen.

Die Analyse möglicher Einflussfaktoren mittels ANCOVA für Endotamponade, Alter, Geschlecht, sphärisches Äquivalent, Linsenstatus und Anzahl der eingesetzten drucksenkenden Augentropfen ergab keinen statistisch signifikanten Einfluss auf den IOD. Jedoch konnte ein signifikanter Unterschied für den Faktor Vitrektomgröße berechnet werden. Hier zeigte sich ein statistisch signifikanter IOD-Abfall im Zeitraum 6-12 Monate für die 20-GOperation, während die 23-G-Operation nahezu stabile IOD-Werte zeigte. Diesen Unterschied erklärten wir uns am ehesten durch eine Steigerung des uveoskleralen Abflusses, welcher bei 
20-G-Vitrektomie durch die bessere Abtragung des Glaskörpers in der Peripherie erreicht werden könnte. 


\section{Anhang}

\subsection{Ethikkomission-Erlaubnis}

\section{IJNIVERSITATSMEDIZIN $=1 \mathrm{WG}$
GOTTINGIFN $=\mathbf{W G}$}

veras per F $9 x: 6787$

$01.10 .2013 \mathrm{br}$ - zi Daturn

Herrn

Prof. Dr. med. Hans Hoerauf

Klinik für Augentei <unde

Rotert-Koch-Straße 40

Nachrichtlich an Frau Julia Charlotte Getest per E-Vail: julia.gebest @agmail.com

Antragsnummer $\quad$ DOK_107_2013 (bitte atats angeben)

Studientitel: $\quad$ Einflusa cor Vitrektornie bai Wakulacrkmangen auf den Augeninnerdiuck.

Antragsteller: $\quad$ Frof. Dr. med. Hans Hoeraut, Klnik für A.ugenheilkunde. UBAG

Doktorandin: Julia Charlotte Getest

Sehr geenter Herr Prof. Dr. Hoerauf, sehr geehrte Damen und Herren,

nacn der uns vorgelegten Kurzdarstellung des cben genannten Promctionswrojektes sollen ausschlia[slich die an do UMG verliegenden Behandlungsdaten auagewertet werden. Eine derartige Auswertung is: ontsorechend den allgemeinen Vertragabedingungen fur die Behandlung an der UMG und ontsprochend dem nicdersächsischen

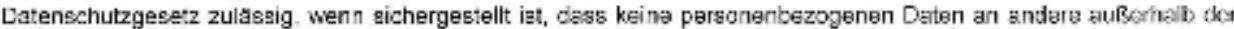
behandelnden El nichtung weitergegeben werden

Gegen die oben genannte Doktorarbeit bestchen damit keine Bedenken.

Auf folgendes mochten wir hirweisen:

1. Z̈nderungen in Vorgehen tai diesan Projekt können einen Antrag an cie Ethik-Kommissicn erfordertich

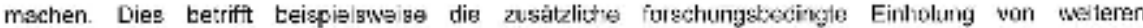
Infonmetionen bei Pabienten, deren Angeherigen oder extemen Xrzien. Dies betrifh atser / B, auch weitere Untersuchungen an biclogischen Batorialien, die an der UMG gelagert sind.

2. Wir mochten bitten. dass dia Coktorandinüicr Daktorandin ein Verschwiegenheitserklårung genau liest, mi:

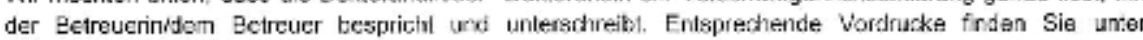
httrimw roxtrs mot.uni-gcctingen.de/Roxtraindex.sepx. Diese verschwiegenheitserkesung sollte fur 10 Jahre bei den Unterlaggen der Ealreuer autbewahrt werden. Bei alen Unklarheiten bezuglich des Datenschutzes wenden \$ie s ch bitle an den Datenschutzbeguftragten der UPS. Herrn Dr. Deler.

3 Wir geten davon aus, dasa projektspezifisch kcinc sersonenbezcgcnon Daten gespeichert werden. Aus Datenschutzgranden müsen wir auch dringend davor abraton, etwa in Anhlëngen zur Doktorarbeit listen der Einzel:Jaten zu thren, und zwar dies auch wenn dic Daton naturtich ohne persorienbezuljene Inform alicnen wie Name, Anschnift und Oeburtadatum geaps chert sind.

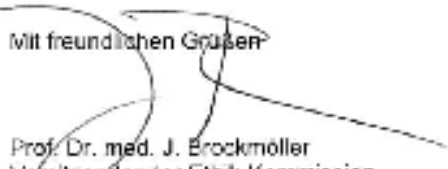

Voksikender der Ethik-Kommission 


\section{Literaturverzeichnis}

Ahn SJ, Woo SJ, Ahn J, Park KH (2012): Comparison of postoperative intraocular pressure changes between 23-gauge transconjunctival sutureless vitrectomy and conventional 20-gauge vitrectomy. Eye (Lond) 26, $796-802$

Altaweel M, Ip M (2003): Macular hole: improved understanding of pathogenesis, staging, and management based on optical coherence tomography. Semin Ophthalmol 18, 58-66 Anderson NG, Fineman MS, Brown GC (2006): Incidence of intraocular pressure spike and other adverse events after vitreoretinal surgery. Ophthalmology $\underline{113}, 42-47$

Augustin AJ, Offermann I (2007): Möglichkeiten und Grenzen der innovativen Vitrektomiesysteme. Klin Monbl Augenheilkd 224, 707-715

Aung KZ, Makeyeva G, Adams MK, Chong EW-T, Busija L, Giles GG, English DR, Hopper J, Baird PN, Guymer RH et al. (2013): The prevalence and risk factors of epiretinal membranes: the Melbourne Collaborative Cohort Study. Retina 33, 1026-1034

Benz MS, Escalona-Benz EM, Murray TG, Eifrig CWG, Yoder DM, Moore JK, Schiffman JC (2004): Immediate postoperative use of a topical agent to prevent intraocular pressure elevation after pars plana vitrectomy with gas tamponade. Arch Ophthalmol 122, 705-709 Blondeau P, Tetrault JP, Papamarkakis C (2001): Diurnal variation of episcleral venous pressure in healthy patients: a pilot study. J Glaucoma $\underline{10}, 18-24$

Brooks H (2000): Macular hole surgery with and without internal limiting membrane peeling. Ophthalmology 107, 1939-1948

Brubaker RF (1991): Flow of aqueous humor in humans [The Friedenwald Lecture]. Invest Ophthalmol Vis Sci $\underline{32}, 3145-3166$

Bu SC, Kuijer R, Li XR, Hooymans J, Los LI (2014): Idiopathic epiretinal membrane. Retina $\underline{34}, 2317-2335$

Casuso LA, Scott IU, Flynn HW, Gass JM, Smiddy WE, Lewis ML, Schiffman J (2001):

Long-term follow-up of unoperated macular holes. Ophthalmology 108, 1150-1155

Chang S (2006): LXII Edward Jackson lecture: open angle glaucoma after vitrectomy. Am J Ophthalmol 141, 1033-1043

Chen CJ (1998): Glaucoma after macular hole surgery. Ophthalmology 105, 94-100 
Chen PP, Thompson JT (1997): Risk factors for elevated intraocular pressure after the use of intraocular gases in vitreoretinal surgery. Ophthalmic Surg Lasers $\underline{28}, 37-42$

Desai UR, Alhalel AA, Schiffman RM, Campen TJ, Sundar G, Muhich A (1997): Intraocular pressure elevation after simple pars plana vitrectomy. Ophthalmology 104, 781-786

Dithmar S (2005): Makulaforamen Überblick und aktuelle chirurgische Konzepte.

Ophthalmologe 102, 191-207

Eckardt C (2005): Transconjunctival sutureless 23-gauge vitrectomy. Retina 25, 208-211

Eckardt C, Eckardt U, Groos S, Luciano L, Reale E (1997): Entfernung der Membrana limitans interna bei Makulalöchern Klinische und morphologische Befunde *. Ophthalmologe $\underline{94}, 545-551$

Ethier CR (2002): The inner wall of Schlemm's canal. Exp Eye Res 74, 161-172

Fine HF, Iranmanesh R, Iturralde D, Spaide RF (2007): Outcomes of 77 consecutive cases of 23-gauge transconjunctival vitrectomy surgery for posterior segment disease. Ophthalmology $\underline{114}, 1197-1200$

Foos RY (1977): Vitreoretinal juncture; epiretinal membranes and vitreous. Invest Ophthalmol Vis Sci $\underline{16}, 416-422$

Foster WJ (2008): Vitreous Substitutes. Expert Rev Ophthalmol $\underline{3}$, 211-218

Framme C, Klotz S, Wolf-Schnurrbusch UEK, Wiedemann P, Wolf S (2012): Intraocular pressure changes following 20G pars-plana vitrectomy. Acta Ophthalmol 90, 744-749

Fraser-Bell S, Guzowski M, Rochtchina E, Wang JJ, Mitchell P (2003): Five-year cumulative incidence and progression of epiretinal membranes. Ophthalmology $\underline{110}, 34-40$

Fraser-Bell S, Ying-Lai M, Klein R, Varma R (2004): Prevalence and Associations of Epiretinal Membranes in Latinos: The Los Angeles Latino Eye Study. Invest Ophthalmol Vis $\operatorname{Sci} \underline{45}, 1732$

Freeman WR (1993): Vitrectomy Surgery for Full-thickness Macular Holes. Am J Ophthalmol 116, 233-235

Fujii GY, Juan E de, Humayun MS, Chang TS, Pieramici DJ, Ng E, Barnes A, Wu SL, Sommerville DN (2002a): A new 25-gauge instrument system for transconjunctival sutureless vitrectomy surgery. Ophthalmology $\underline{109}, 1807-1812$ 
Fujii GY, Juan E de, Humayun MS, Pieramici DJ, Chang TS, Barnes A, Kent D (2002b):

Initial experience using the transconjunctival sutureless vitrectomy system for vitreoretinal surgery. Ophthalmology $\underline{109}, 1814-1820$

Fujikawa M, Sawada O, Kakinoki M, Sawada T, Kawamura H, Ohji M (2014): Long-term intraocular pressure changes after vitrectomy for epiretinal membrane and macular hole. Graefes Arch Clin Exp Ophthalmol 252, 389-393

Gass JD (1988): Idiopathic senile macular hole. Its early stages and pathogenesis. Arch Ophthalmol 106, 629-639

Gass JM (1995): Reappraisal of biomicroscopic classification of stages of development of a macular hole. Am J Ophthalmol 119, 752-759

Goel M, Picciani RG, Lee RK, Bhattacharya SK (2010): Aqueous humor dynamics: a review. Open Ophthalmol J $\underline{4}, 52-59$

Goldmann H, Schmidt T (1957): Über Applanationstonometrie. Ophthalmologica 134, $221-$ 242

Grehn F: Augenheilkunde, 30., überarbeitete und aktualisierte Auflage; Springer Medizin Verlag, Heidelberg 2008

Gupta OP, Ho AC, Kaiser PK, Regillo CD, Chen S, Dyer DS, Dugel PU, Gupta S, Pollack JS (2008): Short-term outcomes of 23-gauge pars plana vitrectomy. Am J Ophthalmol 146, 193 197

Han DP, Lewis H, Lambrou FH, Mieler WF, Hartz A (1989): Mechanisms of intraocuular pressure elevation after pars plana vitrectomy. Ophthalmology $\underline{96}, 1357-1362$

Hasegawa Y, Okamoto F, Sugiura Y, Okamoto Y, Hiraoka T, Oshika T (2012): Intraocular pressure elevation in the early postoperative period after vitrectomy for rhegmatogenous retinal detachment. Jpn J Ophthalmol 56, 46-51

Hikichi T, Trempe CL (1995): Risk of bilateral idiopathic preretinal macular fibrosis. Eye $\underline{9}$, $64-66$

Hikichi T, Yoshida A, Akiba J, Trempe CL (1995): Natural outcomes of stage 1, 2, 3, and 4 idiopathic macular holes. Br J Ophthalmol $\underline{79}$, 517-520

Ho AC, Guyer DR, Fine SL (1998): Macular Hole. Surv Ophthalmol 42, 393-416 
Holekamp NM, Shui Y-B, Beebe D (2005): Vitrectomy surgery increases oxygen exposure to the lens: a possible mechanism for nuclear cataract formation. Am J Ophthalmol $\underline{139}, 302-$ 310

Holekamp NM, Shui Y-B, Beebe D (2006): Lower intraocular oxygen tension in diabetic patients: possible contribution to decreased incidence of nuclear sclerotic cataract. Am J Ophthalmol 141, 1027-1032

Horio N, Horiguchi M (2004): Effect on visual outcome after macular hole surgery when staining the internal limiting membrane with indocyanine green dye. Arch Ophthalmol $\underline{122}$, 992-996

Isomae T, Sato Y, Shimada H (2002): Shortening the duration of prone positioning after macular hole surgery- comparison between 1-week and 1-day prone positioning. Jpn J Ophthalmol $\underline{46}, 84-88$

Iwanoff A (1865): Beiträge zur normalen und pathologischen Anatomie des Auges. Graefes Arch Clin Exp Ophthalmol 11, 135-170

Johnson M (2006): What controls aqueous humour outflow resistance? Exp. Eye Res. $\underline{82}$, $545-557$

Kelly NE, Wendel RT (1991): Vitreous surgery for idiopathic macular holes. Results of a pilot study. Arch Ophthalmol 109, 654-659

Ki-I Y, Yamashita T, Uemura A, Sakamoto T (2013): Long-term intraocular pressure changes after combined phacoemulsification, intraocular lens implantation, and vitrectomy. Jpn J Ophthalmol $\underline{57}, 57-62$

Klein R, Klein BE, Wang Q, Moss SE (1994): The epidemiology of epiretinal membranes. Trans Am Ophthalmol Soc 92, 403-430

Kohno RI, Hata Y, Kawahara S, Kita T, Arita R, Mochizuki Y, Aiello LP, Ishibashi T (2009): Possible contribution of hyalocytes to idiopathic epiretinal membrane formation and its contraction. Br J Ophthalmol 93, 1020-1026

Koreen L, Yoshida N, Escariao P, Niziol LM, Koreen IV, Musch DC, Chang S (2012): Incidence of, risk factors for, and combined mechanism of late-onset open-angle glaucoma after vitrectomy. Retina $\underline{32}, 160-167$ 
Krishnan R, Tossounis C, Fung Yang Y (2013): 20-gauge and 23-gauge phacovitrectomy for idiopathic macular holes: comparison of complications and long-term outcomes. Eye (Lond) $\underline{27}, 72-77$

Kritzenberger M, Junglas B, Framme C, Helbig H, Gabel V-P, Fuchshofer R, Tamm ER, Hillenkamp J (2011): Different collagen types define two types of idiopathic epiretinal membranes. Histopathology $\underline{58}, 953-965$

Lalezary M, Kim SJ, Jiramongkolchai K, Recchia FM, Agarwal A, Sternberg P (2011): Longterm trends in intraocular pressure after pars plana vitrectomy. Retina 31, 679-685

Lalezary M, Shah RJ, Reddy RK, Kammer JA, Kuchtey RW, Joos KM, Cherney EF, Recchia FM, Kim SJ (2014): Prospective Retinal and Optic Nerve Vitrectomy Evaluation (PROVE) study: twelve-month findings. Ophthalmology 121, 1983-1989

Lange CAK, Membrey L, Ahmad N, Wickham L, Maclaren RE, Solebo L, Xing W, Bunce C, Ezra E, Charteris D et al. (2012): Pilot randomised controlled trial of face-down positioning following macular hole surgery. Eye (Lond) $\underline{26}$, 272-277

Liu H, Fan S, Gulati V, Camras LJ, Zhan G, Ghate D, Camras CB, Toris CB (2011): Aqueous humor dynamics during the day and night in healthy mature volunteers. Arch Ophthalmol $\underline{129}, 269-275$

Lott MN, Manning MH, Singh J, Zhang H, Singh H, Marcus DM (2008): 23-gauge vitrectomy in 100 eyes: short-term visual outcomes and complications. Retina $\underline{28}, 1193-1200$ Luk FOJ, Kwok AKH, Lai TYY, Lam DSC (2009): Presence of crystalline lens as a protective factor for the late development of open angle glaucoma after vitrectomy. Retina $\underline{29}$, $218-224$

Lütjen-Drecoll E (1999): Functional morphology of the trabecular meshwork in primate eyes. Prog Retin Eye Res 18, 91-119

McCannel CA, Ensminger JL, Diehl NN, Hodge DN (2009): Population-based incidence of macular holes. Ophthalmology 116, 1366-1369

Mester V, Kuhn F (2000): Internal limiting membrane removal in the management of fullthickness macular holes. Am J Ophthalmol 129, 769-777

Meyer C, Lang G (2010): Nachruf für Robert Machemer (1933 - 2009). Klin Monatsbl Augenheilkd 227, 510-511 
Mi CW, Thompson JT (2015): Long-term follow-up of intraocular pressure after vitrectomy in eyes without preexisting glaucoma. Retina $\underline{35}, 2543-2551$

Misra A, Ho-Yen G, Burton RL (2009): 23-gauge sutureless vitrectomy and 20-gauge vitrectomy: a case series comparison. Eye (Lond) $\underline{23}$, 1187-1191

Mitchell P, Smith W, Chey T, Wang JJ, Chang A (1997): Prevalence and Associations of Epiretinal Membranes. Ophthalmology 104, 1033-1040

Müller M, Geerling G, Zierhut M, Klink T (2010): Glaukom und Netzhautchirurgie. Ophthalmologe 107, 419-426

Nau CB, Malihi M, McLaren JW, Hodge DO, Sit AJ (2013): Circadian variation of aqueous humor dynamics in older healthy adults. Invest Ophthalmol Vis Sci $\underline{54}, 7623-7629$

Noyes HD (1871): Detachment of Retina with Laceration at Macula. Trans Am Ophthalmol Soc $1,128-129$

Ohlmann A, Tamm ER (2002): Die Rolle von Myocilin bei der Pathogenese des primaren Offenwinkelglaukoms. Ophthalmologe 99, 672-677

Parapuram SK, Chang B, Li L, Hartung RA, Chalam KV, Nair-Menon JU, Hunt DM, Hunt RC (2009): Differential effects of TGFbeta and vitreous on the transformation of retinal pigment epithelial cells. Invest Ophthalmol Vis Sci 50, 5965-5974

Park DW, Dugel PU, Garda J, Sipperley JO, Thach A, Sneed SR, Blaisdell J (2003): Macular pucker removal with and without internal limiting membrane peeling: Pilot study.

Ophthalmology $\underline{110}, 62-64$

Parravano M, Giansanti F, Eandi CM, Yap YC, Rizzo S, Virgili G (2015): Vitrectomy for idiopathic macular hole. Cochrane Database Syst Rev $\underline{5}$, CD009080

Passemard M, Yakoubi Y, Muselier A, Hubert I, Guillaubey A, Bron AM, Berrod JP, Creuzot-Garcher C (2010): Long-term outcome of idiopathic macular hole surgery. Am J Ophthalmol $\underline{149}, 120-126$

Reiss GR, Lee DA, Topper JE, Brubaker RF (1984): Aqueous humor flow during sleep. Invest Ophthalmol Vis Sci $\underline{25}, 776-778$

Rizzo S, Belting C, Genovesi-Ebert F, Di Bartolo E (2010): Incidence of retinal detachment after small-incision, sutureless pars plana vitrectomy compared with conventional 20-gauge vitrectomy in macular hole and epiretinal membrane surgery. Retina (Philadelphia, Pa.) $\underline{30}$, 1065-1071 
Saccà SC, Pascotto A, Camicione P, Capris P, Izzotti A (2005): Oxidative DNA damage in the human trabecular meshwork: clinical correlation in patients with primary open-angle glaucoma. Arch Ophthalmol $\underline{123}, 458-463$

Sachsenweger M: Augenheilkunde (Duale Reihe), 2., vollst. überarb. und erw. Auflage; Thieme, Stuttgart 2003

Schechet SA, DeVience E, Thompson JT (2016): The effect of internal limiting membrane peeling on idiopathic epiretinal membrane surgery, with a review of the literature. Retina $\underline{37}$, $873-880$

Schweinitz GE de (1904): Concerning certain non-traumatic perforations of the macula lutea. Trans Am Ophthalmol Soc 10, 228-237

Shah S (2000): Accurate intraocular pressure measurement - the myth of modern ophthalmology? Ophthalmology $\underline{107}, 1805-1807$

Siegfried CJ, Shui Y-B, Holekamp NM, Bai F, Beebe DC (2010): Oxygen distribution in the human eye: relevance to the etiology of open-angle glaucoma after vitrectomy. Invest Ophthalmol Vis Sci $\underline{51}, 5731-5738$

Singh CN, Iezzi R, Mahmoud TH (2010): Intraocular pressure instability after 23-gauge vitrectomy. Retina 30, 629-634

Sippy BD, Engelbrecht NE, Hubbard GB, Moriarty SE, Jiang S, Aaberg TM, Grossniklaus HE, Sternberg P (2001): Indocyanine green effect on cultured human retinal pigment epithelial cells: Implication for macular hole surgery. Am J Ophthalmol 132, 433-435

Smiddy WE, Flynn HW (2004): Pathogenesis of macular holes and therapeutic implications. Am J Ophthalmol 137, 525-537

Snead DRJ, Cullen N, James S, Poulson AV, Morris AHC, Lukaris A, Scott JD, Richards AJ, Snead MP (2004): Hyperconvolution of the inner limiting membrane in vitreomaculopathies. Graefes Arch Clin Exp Ophthalmol 242, 853-862

Su J, Liu X, Zheng L, Cui H (2015): Vitrectomy with internal limiting membrane peeling vs no peeling for Macular Hole-induced Retinal Detachment (MHRD): a meta-analysis. BMC Ophthalmol $\underline{15}, 62$

The Eye Disease Case-Control Study Group (1994): Risk Factors for Idiopathic Macular Holes. Am J Ophthalmol 118, 754-761 
Thompson JT, Sjaarda RN, Glaser BM, Murphy RP (1996): Increased intraocular pressure after macular hole surgery. Am J Ophthalmol $\underline{121}, 615-622$

Toris CB: Aqueous Humor Dynamics and Intraocular Pressure Elevation. In: Shaarawy T, Sherwood MB, Hitchings RA, Crowston JG (Hrsg.): Glaucoma: Medical diagnosis and therapy. 2. Auflage; Elsevier Saunders, London u. a. 2015, 47-56

Toris CB, Yablonski ME, Wang Y-L, Camras CB (1999): Aqueous humor dynamics in the aging human eye. Am J Ophthalmol $\underline{127}, 407-412$

Toyokawa N, Kimura H, Matsumura M, Kuroda S (2015): Incidence of late-onset ocular hypertension following uncomplicated pars plana vitrectomy in pseudophakic eyes. Am J Ophthalmol $\underline{159}, 727-732$

True Gabelt BA, Kaufmann PL: Production and flow of aqueous humor. In: Kaufman PL, Alm A, Adler FH, Levin LA (Hrsg.): Adler's Physiology of the Eye. 11. Auflage; Saunders Elsevier, Edingburgh u. a. 2011, 274-307

Vagaja NN, Chinnery HR, Binz N, Kezic JM, Rakoczy EP, McMenamin PG (2012): Changes in murine hyalocytes are valuable early indicators of ocular disease. Invest Ophthalmol Vis Sci $\underline{53}, 1445-1451$

Viggiano SR, Koskela TK, Klee GG, Samples JR, Arnce R, Brubaker RF (1994): The Effect of Melatonin on Aqueous Humor Flow in Humans during the Day. Ophthalmology 101, 326331

Wakely L, Rahman R, Stephenson J (2012): A comparison of several methods of macular hole measurement using optical coherence tomography, and their value in predicting anatomical and visual outcomes. Br J Ophthalmol 무, 1003-1007

Weinberg RS, Peyman GA, Huamonte FU (1976): Elevation of intraocular pressure after pars plana vitrectomy. Graefes Arch Klin Ophthalmol 200, 157-161

Wimpissinger B, Kellner L, Brannath W, Krepler K, Stolba U, Mihalics C, Binder S (2008): 23-Gauge versus 20-gauge system for pars plana vitrectomy: a prospective randomised clinical trial. Br J Ophthalmol $\underline{92}, 1483-1487$

Wu L, Berrocal MH, Rodriguez FJ, Maia M, Morales-Canton V, Figueroa M, Serrano M, Roca JA, Arevalo JF, Navarro R et al. (2014): Intraocular pressure elevation after uncomplicated pars plana vitrectomy: results of the Pan American Collaborative Retina Study Group. Retina 34, 1985-1989 
Yamamoto K, Iwase T, Terasaki H (2016): Long-Term Changes in Intraocular Pressure after Vitrectomy for Rhegmatogenous Retinal Detachment, Epi-Retinal Membrane, or Macular Hole. PLoS One $\underline{11}$, e0167303

Yamashita T, Uemura A, Kita H, Sakamoto T (2007): Intraocular pressure after intravitreal injection of triamcinolone acetonide following vitrectomy for macular edema. J Glaucoma $\underline{16}$, $220-224$

Yu AL, Brummeisl W, Schaumberger M, Kampik A, Welge-Lussen U (2010): Vitrectomy does not increase the risk of open-angle glaucoma or ocular hypertension--a 5-year follow-up. Graefes Arch Clin Exp Ophthalmol 248, 1407-1414

Zhang J, Li Y, Zhao X, Yu X, Lu L (2015): Comparison of Clinical Features After 20-Gauge Vitrectomy Versus 23-Gauge Vitrectomy. Asia Pac J Ophthalmol 4, 367-370 


\section{Danksagung}

Zunächst möchte ich mich bei Herrn Professor Hoerauf als Abteilungsleiter für die Bereitstellung des Themas und die Ermöglichung der Arbeit bedanken.

Großer Dank gebührt meinem Doktorvater Herrn PD Dr. med. Christian van Oterendorp, der gemeinsam mit mir die Fragestellung gut definierte und mir bei Unklarheiten immer zur Seite stand. Dank der hilfreichen Ratschläge und der guten Betreuung konnte die Arbeit nach Verzögerung durch meinen beruflichen Einstieg einen raschen Abschluss finden. Ganz besonders danke ich für die guten Tipps und die schnelle Korrektur.

Des Weiteren danke ich Herrn Sebastian Pfeiffer, der für mich die erste statistische Auswertung der Rohdaten aus dem Archiv vorgenommen hat, was die Auswertung erleichtert hat. Auch den Mitarbeitern des Archivs der UMG danke ich für die Bereitstellung der Datentabelle und das Heraussuchen und Einsortieren der genutzten Akten.

Zuletzt danke ich auch meinem Mann, der mir an seinen freien Abenden liebevoll und mit viel Geduld half, Grafiken zu erstellen und die statistische Auswertung zu perfektionieren. 